\title{
Huntington Study Group Abstracts 2020
}

\author{
Published online: 20 October 2020 \\ (C) The American Society for Experimental NeuroTherapeutics, Inc. 2020, corrected publication 2020
}

1

\section{Motor Performance Provides Insights into Cognition and Pain in Persons with Huntington's Disease}

\section{Erin Edwards, Nora Fritz*}

Leslie Dunnington*, Erin Furr Stimming, Amber McCarthy, Jorge Patino-Murillas, Chloe Denham

Background: Huntington's disease (HD) results in motor, cognitive, and psychosocial impairments. Little is known about the specific relationships among cognitive, psychosocial, and simple and complex motor task performance.

Objective: The objective of this pilot study was to explore performance on motor, cognitive, and behavioral measures between individuals with HD and healthy controls, and to determine the relationships among specific domains of cognitive function and motor function and pain.

Methods: Individuals with HD and healthy controls performed a battery of cognitive, motor, and survey measures (i.e., fall reports, quality of life, and pain) in a single session. We examined differences between individuals with HD and controls, as well as relationships among motor, cognitive, and survey measures.

Results: Four individuals with HD (mean(SD) age: 62.5(14.5); symptom duration: 5.8(7.1); 1 female) and six healthy controls (age: 50.7(9.7); 6 females) completed this study. There was no significant difference between groups for age, gender, or years of education. As expected, individuals with HD performed significantly worse on motor and cognitive testing, as evidenced by slower walking $(p=0.019)$, poorer lower extremity coordination $(p=0.038)$, poorer working memory $(p=0.010)$, and worse pain severity $(p=0.010)$. Poorer working memory was significantly related to poorer performance timed walking $(r=-0.705 ; p=$ $0.023)$; lower extremity coordination $(r=-0.657 ; p=0.039)$; and dual-tasks $(r=-0.760 ; p=0.011)$, as well as reports of more falls $(r=-0.709 ; p=0.022)$. Poorer cognitive processing speed was specifically related to worse dual-task performance $(r=-0.733$; $p=0.016$ ). Worse pain severity was significantly related to slower timed walking $(r=0.679 ; p=0.031)$; poorer lower extremity coordination $(r=0.743 ; p=0.014)$; and worse dual-task performance $(r=0.731 ; p=0.016)$, as well as poorer working memory $(r>-$ $0.811 ; p<0.004)$ and reports of more falls $(r=0.713 ; p=0.021)$. Conclusions: Individuals with HD experience declines in motor and cognitive performance, as well as increased pain, compared to healthy controls. Quick and easily administered motor tests, such as timed walking and lower extremity coordination, as well as surveys to assess pain, may be useful in determining underlying cognitive impairments in working memory and processing speed. Assessment of these factors may help clinicians to tailor rehabilitation protocols to improve outcomes in persons with HD.

\section{2}

Predictive Genetic Testing for Huntington's Disease During the COVID-19 Pandemic

Leslie Dunnington*, Erin Furr Stimming, Amber McCarthy, Jorge Patino-Murillas, Chloe Denham

University of Texas, Houston, Houston, TX, USA (all authors)

Background: The 2016 Huntington's Disease Society of America predictive testing protocol outlines best practices in providing predictive genetic testing for Huntington's disease. This includes at least one in-person, pretest genetic counseling visit, and an inperson result disclosure. Due to the COVID-19 pandemic of 2020, many healthcare providers have made necessary adjustments to their patient care including offering options such as telemedicine or remote visits. This may have introduced barriers for patients seeking predictive genetic testing or who are in the process of predictive genetic testing.

Methods: A survey was distributed to genetic counselors who provide genetic counseling for the Huntington's disease population that was aimed at assessing their clinic's response to the COVID-19 pandemic. This survey attempted to describe the predictive and confirmatory genetic testing protocols utilized by clinics that offer this nation-wide, as well as challenges in the implementation of these protocols with regard to COVID-19 and practice response to these challenges.

Results: A total of 25 responses were received from genetic counselors and geneticists. The majority $(94.7 \%)$ of these providers always implement the HDSA predictive testing protocol with a mean of 2.53 in-person visits required before a genetic test is ordered. Due to the COVID-19 pandemic, all of these providers have implemented changes such as offering telemedicine appointments, rescheduling, or canceling appointments and limiting in-person appointments to emergency visits only. $63.2 \%$ of respondents indicated that they anticipate a telemedicine option will continue to be available to patients after the COVID-19 pandemic.

Conclusions: This survey addresses the response of genetic counselors who provide genetic testing for HD during the COVID-19 pandemic. Results of the survey indicate that genetic counseling via telemedicine is now being offered and may continue to be an option for patients who are at-risk of HD. Barriers of providing this service were identified, which may help to improve telemedicine genetic counseling for HD for future patients. 
3

Immediate Effects of Treadmill Walking in Individuals with Huntington's Disease and Lewy Body Dementia

Deb Kegelmeyer*1, Sandra Kostyk, Nora Fritz ${ }^{2}$, Sara McKeeman, Douglas Scharre ${ }^{1}$, Gregory Young, Yubo Tan, Robin Schubert, Ralf Reilmann ${ }^{3}$, Anne Kloos ${ }^{1}$

${ }^{1}$ The Ohio State University, Columbus, OH, USA

${ }^{2}$ Wayne State University, Detroit, Michigan, USA

${ }^{3}$ George Huntington Institute, Münster, Germany

Background: Treadmill training may improve gait disorders associated with neurodegenerative diseases. In Parkinson's disease, treadmill training alters gait patterns after one session, and long-term training improves gait parameters, fall risk, and quality of life. This study examined the feasibility of using this intervention for people with Huntington's disease (HD) or Lewy body dementia (LBD).

Methods: Ten individuals with HD, eight individuals with LBD, and ten control individuals walked for $20 \mathrm{~min}$ on a treadmill, starting at a slow comfortable speed and increasing incrementally toward their normal overground speed. Gait measures, Timed Up and Go (TUG) scores, and quantitative measures of motor function (Q-Motor; precision grasp force variability, finger and foot-tapping frequency) were assessed.

Results: Treadmill training is feasible in HD and LBD; although, participants could not initiate treadmill walking at their comfortable overground speeds and only three participants with HD were able to achieve their overground walking speed within the 20 -min session. No changes in gait measures, TUG times, and Q-Motor measures were found among HD and LBD participants following the treadmill walking. Control participants demonstrated significant increases in several gait measures and foot-tap speed frequency after one session.

Conclusions: A 20-min treadmill training session proved safe and feasible for individuals with HD and LBD, but no significant motor effects were found. Longer and more frequent sessions may be needed to see an effect in these disorders. Motor and cognitive impairments associated with these diseases may make them less amenable to the short-term effects of treadmill training.

4

Empowering the Clinical Research Coordinator in Academic Medical Centers

Danielle Buchanan*1, Jody Goldstein ${ }^{2}$, Anna C. Pfalzer ${ }^{1}$, Ya-Chen Lin ${ }^{1}$, Hakmook Kang ${ }^{1}$, Daniel O. Claassen ${ }^{1}$

${ }^{1}$ Vanderbilt University Medical Center, Nashville, TN, USA

${ }^{2}$ University of Rochester, Rochester, NY, USA

Objectives: Historically, a clinical research coordinator (CRC) position was revered as a long-term career with many staying in the position for 15-30 years. However, the culture of the position has shifted to being short-term with a high turnover rate. The consequences of low retention are felt throughout the clinical research setting: it causes unnecessary stress to patients, increases the burden on current employees and the employer who must devote time to training, and involves substantial administrative resources to identify and hire new employees. The cause behind the cultural shift toward CRCs as a temporary position is unclear, and there is little research on the cause for low retention and high turnover among these clinical workers. The identification of factors significantly associated with longer retention would be beneficial for the infrastructure of clinical research.
Methods: An online survey containing 13 multiple-choice or open-ended and 32 Likert scale questions was distributed to previous and current clinical research coordinators using RedCap. The questionnaires were self-administered and completed between October 2017 and September 2018. A cluster analysis identified groups of questions with similar response trends and those predictors were further investigated for their association with retention using logistic regression models.

Results: Twelve previous and 79 current CRCs between 22 and 80 years of age completed the study $(n=85)$. Fifty-eight participants worked in a hospital setting with six in academia and 21 in a private research setting. Seventy-six participants were female, and the age range was 22-80 years of age. From the 15 potential predictors analyzed, there were nine significant predictors of retention, including salary, knowledge of the job role, and job level, as well as several predictors relating to the role of the Principal Investigator. Good salary, higher job level and greater respect, collaboration, and engagement from the PI were significantly associated with higher retention. Conversely, the number of trials managed, workload, and opportunity for professional growth were not associated with retention.

Conclusions: Clinical research coordinators who feel respected and engaged by the PI and are adequately compensated are more likely to have higher job satisfaction and retention.

5

Detection of Early Neurodegeneration with Fluid Biomarkers in the HD Young Adult Study

Paul Zeun*1, Rachael I. Scahill ${ }^{1}$, Katherine Osborne-Crowley ${ }^{1}$, Eileanoir B. Johnson ${ }^{1}$, Sarah Gregory ${ }^{1}$, Jessica Lowe ${ }^{1}$, Akshay Nair ${ }^{1}$, Marina Papoutsi $^{1}$, Carlos Estevez-Fraga ${ }^{1}$, Kate Fayer ${ }^{1}$, Henny Wellington ${ }^{1}$, Filipe B. Rodrigues ${ }^{1}$, Lauren M. Byrne ${ }^{1}$, Amanda Heslegrave ${ }^{1}$, Cristina Sampaio $^{2}$, Henrik Zetterberg ${ }^{1}$, Hui Zhang ${ }^{1}$, Edward J. Wild ${ }^{1}$, Geraint Rees $^{1}$, Douglas Langbehn ${ }^{3}$, Sarah J. Tabrizi ${ }^{1}$

${ }^{1}$ University College London, London, UK

${ }^{2}$ CHDI Foundation, Los Angeles, CA, USA

${ }^{3}$ University of Iowa, Iowa City, IA, USA

Background: Identifying biomarkers of early neurodegeneration in HD is crucial to future treatment strategies that aim to intervene early in the disease process before clinical function is impaired. We studied a series of biofluid biomarkers to evaluate how early neurodegeneration can be detected in HD and which biomarkers may be most sensitive at this stage.

Methods: The HD young adult study (HD-YAS) recruited 64 premanifest gene carriers (PreHD) $>18$ years from predicted onset based on age-CAG calculations and 67 matched controls. All but one had plasma, while 109 underwent optional CSF collection. Total huntingtin, mHTT, Neurofilament light (NfL), YKL-40, total tau, neurogranin, interleukin (IL)-6, IL-8, glial fibrillary acid protein (GFAP), and ubiquitin carboxyl-terminal hydrolase L1 (UCHL1) were measured in CSF; NfL, total tau, and GFAP were also measured in plasma. For each biomarker, we evaluated group differences and age-cag effects in preHD. We corrected for multiple comparisons using the false discovery rate (FDR). We also performed receiver operating characteristic (ROC) area under the curve (AUC) analysis for any biomarkers showing group differences and modeled NfL trajectories for CAG repeats 40-45 from 20 to 70 years by including HD-CSF and HD-YAS data.

Results: Gene carriers were, on average, 24 years from predicted disease onset. Only $40 \%$ of gene carriers had mHTT levels above the lower limit of quantification. There were significant differences between preHD and controls for CSF NfL (FDR < 0.0001), plasma NfL (FDR =0.01), 
and CSF YKL-40 (FDR =0.03). No other analytes showed significant group differences. Only CSF NfL showed a significant association with age-cag (FDR <0.0001). ROC analysis showed CSF NfL had a superior discriminatory ability (AUC 0.79) compared to plasma NfL (AUC 0.65) and YKL-40 (AUC 0.64). NfL curves demonstrated a sigmoid trajectory with CSF NfL levels crossing the upper 95th prediction interval of controls at an earlier age than plasma NfL.

Conclusions: NfL is a sensitive measure of early neurodegeneration in preHD 24 years from predicted onset. CSF NfL appears to be more sensitive than plasma NfL at this early stage and may be useful in future premanifest trials for enrichment and measuring treatment response. Elevations in YKL-40 suggest a measurable astrocytic response to early neurodegeneration.

\section{Clinical Practice Guidelines for Physical Therapy for Huntington's} Disease

Anne Kloos*1, Nora Fritz ${ }^{2}$, Lori Quinn ${ }^{3}$, Ashwini K. Rao ${ }^{3}$, Monica Busse $^{4}$, Deb Kegelmeyer ${ }^{1}$, members of the Huntington Study Group Rehabilitation Therapists Working Group

${ }^{1}$ The Ohio State University, Columbus, OH, USA

${ }^{2}$ Wayne State University, Detroit, MI, USA

${ }^{3}$ Columbia University, New York, NY, USA

${ }^{4}$ Cardiff University

Background: A growing number of studies have been published on physical therapy and exercise interventions for people with Huntington's disease (HD) in the past decade. In 2016, a guideline development group (GDG) was formed with support from the Huntington Study Group, European Huntington Disease Network, and the Griffin Foundation to develop a clinical practice guideline for physical therapy interventions in HD.

Methods: The GDG conducted a mixed-methods systematic review of 23 quantitative and three qualitative studies using Joanna Briggs Institute (JBI) methodology and expert opinion. The review suggests that physical therapy interventions may be beneficial for mitigating motor impairments and activity limitations in individuals with HD. The GDG subsequently provided specific recommendations to guide clinicians in translating the findings into practice.

Results: Six action statements with different JBI grades of recommendation were developed. Aerobic exercise, alone or in combination with resistance training, to improve fitness and motor function, and supervised one-on-one gait training to improve spatiotemporal measures of gait were strongly recommended. Weak recommendations were made for the use of balance exercises to improve balance, and for breathing exercises, including inspiratory and expiratory training, to improve respiratory function. There was expert consensus for the use of an individualized postural control training program; the use of positioning devices to optimize posture; and the use of positioning devices, seating adaptations, and caregiver training in late-stage HD. Decision trees to provide guidance for therapists are being developed for both assessment and intervention based on the treatment-based classifications and results of the systematic review.

Conclusions: While there is still a need for rigorous evaluation of physical therapy interventions, these guidelines provide evidence in support of aerobic exercise and supervised gait training. Facilitators of exercise participation include shared goal setting, individualized treatment plans, group therapy, strategies to improve adherence, and involvement of a caregiver.
Abnormal Rate of Weight Gain in Adults at Risk for Huntington's Disease

Amy C. Ogilvie*, Peg C. Nopoulos, Jordan L. Schultz

The University of Iowa, Iowa City, IA, USA (all authors)

Background: Lower body mass indexes (BMIs) have been identified cross-sectionally in premanifest Huntington's disease (preHD) patients, and weight loss is a common symptom in manifest stage disease. The objective of this study was to determine if the patterns of weight gain or weight loss differ between preHD participants and non-HD controls and whether those patterns are associated with CAG length.

Methods: Using data from participants enrolled in the Enroll-HD study who were 18 to 65 years old, 1837 preHD participants (CAG 40-59) were compared to 2109 non-HD controls $(\mathrm{CAG}<36)$. Participants in the preHD group were required to have a diagnostic confidence level less than four, a total motor score of 10 or less, and a total functional capacity score of 13. All Enroll-HD sites have approval from their local ethics and institutional review boards, and participants are consented prior to participation. Linear mixed-effects regression models with a random intercept for each participant ID were used to investigate the non-linear relationships between BMI and age, CAG length, and CAG-Age product (CAP) score. For the primary analysis, non-linear regression models for the association between age and BMI were estimated as a function of genetic mutation status.

Results: PreHD participants had a significantly different rate of weight change than the non-HD controls $(F=11.62, p \leq 0.001)$. PreHD participants gained less weight than the non-HD controls and had an earlier plateau prior to weight loss. The rate of weight change also significantly differed by CAG length $(F=6.24, p=0.002)$ and as a function of CAP score $(F=8.27, p \leq 0.001)$.

Conclusions: This study compared weight trajectories between preHD participants and healthy controls to determine the effect of having the genetic mutation for HD on weight development. The presence of the mutation was associated with an earlier plateau in BMI with age compared to non-HD controls which could be attributed to proximity to motor onset.

8

Effect of Using Antidepressant Medication to Treat Depression in Manifest Huntington's Disease Patients

Amy C. Ogilvie*, Ryan M. Carnahan, Elizabeth A. Chrischilles, Jordan L. Schultz

The University of Iowa, Iowa City, IA, USA (all authors)

Background: Huntington's disease (HD) is a genetic, neurodegenerative disorder that affects an individual's motor, cognitive, and psychiatric performance. Depression is a common psychiatric symptom in the motor-manifest stage of the disease. There is no evidence to guide the treatment of depression in this patient population. Selective serotonin reuptake inhibitors and serotonin-norepinephrine reuptake inhibitors are the antidepressant medications most commonly prescribed. This study aimed to assess the effectiveness of antidepressant medication use on depressive symptoms in manifest HD patients.

Methods: Motor-manifest Huntington's disease patients with depressive symptoms participating in the Enroll-HD study were included in this analysis. All Enroll-HD sites have approval from their local ethics and institutional review boards, and participants are consented prior to participation. Participants who began antidepressant use during the Enroll-HD 
study were matched to non-users to account for differences in age, sex, and disease state. The effect of antidepressant medication on changes in depression severity (Hospital Anxiety and Depression Scale total score) and extent of motor impairment (total motor score), functional impairment (total functional capacity score), and cognitive impairment (Symbol Digit Modality Test) over approximately a one-year time frame were assessed using linear models and linear mixed effect models. Models were adjusted for baseline measures of the outcome variable.

Results: Forty-six antidepressant users and their matched non-user counterparts were analyzed. Initiation of an antidepressant in patients with motor-manifest HD did not significantly change depression scores over 1 year compared to matched patients who never started an antidepressant. There were also no significant differences when assessing change in disease progression measures.

Conclusions: The findings of this study support further research on the effectiveness of antidepressants in HD patients. Due to the small sample size and potential inconsistencies in pharmacotherapy reporting in this study, clinical trials or studies with a larger sample of new antidepressant users should be used to assess the causal effects of antidepressant medications on depressive symptoms and how they affect disease progression.

9

HD Constellation: a Novel Patient-Centered Approach to Enriching Clinical Datasets by Combining Medical Record Chart Review and Enroll-HD

Katherine Belendiuk*1, J.P. Sacksteder, Xerxes Sanii ${ }^{3}$, Eileen Neacy ${ }^{4}$, Jamie Levey ${ }^{4}$, Eli Korner ${ }^{1}$, Richard Houghton, George Yohrling ${ }^{5}$

${ }^{1}$ Genetech Inc., South San Francisco, CA, USA

${ }^{2}$ F. Hoffmann-La Roche Ltd, Basel, Switzerland

${ }^{3}$ PicnicHealth, Los Angeles, CA, USA

${ }^{4}$ CHDI Foundation, Los Angeles, CA, USA

${ }^{5}$ Huntington's Disease Society of America

Introduction: Patient-reported medical histories, including medications, comorbidities, and medical procedures, are often incomplete and inaccurate. Routinely collected Electronic Medical Records (EMR) can be a valuable information source, including treatment patterns, detailed comorbidity profiles, lab values, and healthcare resource use, to provide a more accurate record of factors relevant to disease progression. As Huntington's disease (HD) can require multidisciplinary care, data are often spread across many care facilities and not readily utilizable and accessible. HD Constellation is based on a novel patient-centered approach to EMR data retrieval. Once a participant's permission has been obtained, medical records are retrieved from their healthcare providers and abstracted to create a structured dataset. Retrieved EMR data from medical care in the USA will be matched at an individual level to data from Enroll-HD, an ongoing global observational study and clinical research platform for individuals with HD and their families that collects clinical characteristics, CAG repeat lengths, and clinical assessments (motor, function, behavioral, and cognitive) at baseline and annual visits thereafter. The resulting dataset will provide a longitudinal view of disease course, creating a richer context for studying HD.

Methods: Patients must be participating in Enroll-HD; be 12 years of age or older; sign informed consent or assent for medical record retrieval, abstraction, and linkage; and be confirmed to have the CAG expansion in their huntingtin gene. Records will be collected retrospectively for $\geq$ 7 years for all participants and prospectively for $\leq 3$ years for adults only. Analysis-ready data will be anonymized.

Results: This study will assess feasibility and effectiveness of a patientcentered approach to aggregating and linking multiple sources of data, including EMR and registry data. Baseline demographics and participant characteristics will be presented.

Conclusions: The data source resulting from HD Constellation may deepen understanding of natural history, disease progression, and standards of care for individuals with HD in the USA.

\section{0}

Similarities in Mental Health Symptoms for People with
Huntington's Disease and Non-carriers

Sarah Gunn*, John Maltby, Noora Ovaska-Stafford

University of Leicester, Leicester, UK (all authors)

Objective: Mental health difficulties are common among people with Huntington's disease (HD). However, such difficulties are weakly associated with HD progression, suggesting their causes are multifactorial rather than simply disease related. Evidence suggests that genetically unaffected family members may experience similar levels of mental distress to people with $\mathrm{HD}$, due to systemic stressors and life disruption. These factors may also influence mental wellbeing in people with HD. Accordingly, this study aimed to evaluate quantitative and qualitative similarities and differences between people with HD and genetically unaffected control groups to determine systemic and environmental contributions to HD-related distress.

Methods: Exploratory and confirmatory factor analysis was used to compare the structure of mental distress in 5294 individuals from four groups: manifest or premanifest HD, family controls, and genotype negative. Data were from the Enroll-HD study, using scores from the Problem Behaviors Assessment, the Hospital Anxiety and Depression Scale, and the Snaith Irritability Scale. We then evaluated the consistency of the identified constructs over three annual assessments using analysis of variance.

Results: Four factors consistently emerged across all groups, comprising depression, anxiety, temper, and self-harm; these remained stable across time. People with HD did not report significantly different anxiety scores to control groups. The manifest group reported significantly higher depression, temper, and self-harm than the genotype-negative group, but only differed in some cases from family controls.

Conclusions: The findings suggest greater similarity in the severity and structure of mental health symptoms between people with and without HD than previously believed. This suggests contributions from systemic factors in families affected by HD.

\section{1}

Comparing Coping Mechanisms and Experiences of Young People Prior to and During the COVID-19 Lockdown

Bonnie Hennig-Trestman*1, Catherine Martin ${ }^{1}$, Beth Ann Griffin ${ }^{2}$, Misty Daniel $^{1}$

${ }^{1}$ Huntington's Disease Youth Organization (HDYO), Roanoke, VA, USA

${ }^{2}$ RAND Corporation

Background: Huntington's disease (HD) typically affects individuals $30-50$ years of age. Families may have children impacted by the challenge of growing up in a HD family. Prior to the COVID-19 lockdown these children worked to balance school, work, and caregiving with their own mental health needs. Prior lockdown coping mechanisms included support, visits with loved ones, and attending school/work. These activities have now been limited. This study examines coping mechanisms and experiences of young people impacted by HD during the lockdown. 
Methods: Through social media HD Youth Organization (HDYO) asked people ( $<35$ years old) to share coping experiences during the lockdown. Once an individual replied, he or she was connected to a survey. Survey questions included demographic information; wellbeing, level of demand, and caregiving prior to and during lockdown; and amount of support received from advocacy organizations. Open-ended questions addressed the impact of the lockdown and sought suggestions on how HDYO can support them.

Results: Forty-six people responded. The highest percentage of responders were from Northern America (63\%), followed by the United Kingdom (15\%), Australasia (9\%), Europe (9\%), and South Asia (4\%). The average age of responders was 23.4 years. Perceived well-being prior to the lockdown compared to during the lockdown changed significantly. Most young people (63\%) reported a decrease in well-being. Specifically, prior to the lockdown, $8 \%$ rated well-being as being either "Poor" or "Very Poor." This grew to $37 \%$ during the lockdown. No one reported having "Very Good" well-being during the lockdown compared to $20 \%$ who rated their well-being as "Very Good" prior to the lockdown. When asked if they felt supported by HD advocacy organizations there was a range of responses. Using a Likert scale of 1 to $5(1=$ None and $5=\mathrm{A}$ Lot) $24 \%$ of young people replied with " 1 " or " 2 ." Fifty percent replied with "4" or "5." Replies asking about the lockdown impact included separation from loved ones, feeling isolated, and anxiety/apathy. Ideas to meet the needs of young people included scheduling more live chats (Zoom meetings, Google groups), providing lists of resources, having HDYO staff "check in," and online game nights.

Conclusions: Young people impacted by HD have an added burden due to the COVID-19 lockdown. Support systems are limited, and they are more isolated. Organizations such as HDYO would be a useful resource for creating programs to address the need to build coping skills, increase resiliency, and decrease isolation in young people globally.

\section{2}

Longitudinal Dynamics of Mutant Huntingtin and Neurofilament Light in Huntington's Disease: the Prospective HD-CSF Study

Filipe Brogueira Rodrigues* ${ }^{1}$, Lauren M. Byrne ${ }^{1}$, Rosanna Tortelli ${ }^{1}$, Eileanoir B. Johnson ${ }^{1}$, Peter A. Wijeratne ${ }^{1}$, Marzena Arridge ${ }^{1}$, Enrico De Vita ${ }^{2}$, Daniel C. Alexander ${ }^{1}$, Sarah J. Tabrizi ${ }^{1}$, Scott Schobel $^{3}$, Rachael I. Scahill ${ }^{1}$, Amanda Heslegrave ${ }^{1}$, Henrik Zetterberg ${ }^{1}$, Edward J. Wild ${ }^{1}$

${ }^{1}$ University College London, London, UK

${ }^{2}$ King's College London, London, UK

${ }^{3}$ F. Hoffmann-La Roche Ltd, Basel, Switzerland

Background: Mutant huntingtin (mHTT) and neurofilament light (NfL) have emerged as leading biofluid biomarker candidates for Huntington's disease (HD). However, we lack robust data from repeated sampling of individual HD mutation carriers to define the longitudinal dynamics of these markers.

Methods: We quantified mHTT and NfL in CSF and blood at baseline and 24-months in the prospective HD-CSF study (20 controls, 20 premanifest $\mathrm{HD}, 40$ manifest $\mathrm{HD}$ ). We characterized longitudinal trajectories of each analyte using mixed-effects models and their relationships with disease progression with partial correlations and linear regression. We computed clinical trial simulations to inform clinical trial design.

Results: mHTT and NfL in CSF and plasma all increased over time, had distinct patterns in HD mutation carriers compared with controls, and increased in a manner dependent on HTT CAG count. We defined the age for which each measure departed from normality for a given CAG count. The baseline value of each analyte predicted subsequent clinical progression and brain atrophy better than rate of change in the analytes. Unlike baseline concentrations, rate of change in all analytes did not predict disease status. NfL would require fewer participants per arm than mHTT to run clinical trials as an outcome measure.

Conclusions: NfL is a stronger progression biomarker for HD than mHTT and could be used to inform clinical trial design. CSF mHTT nonetheless possesses prognostic value and will remain an intrinsically valuable pharmacodynamic marker for huntingtin-lowering trials.

\section{3}

Antidepressive Pharmacotherapy in Huntington's Disease: Statistical Analysis from Enroll-HD Dataset

Ilka Hatakeyama*, Paddy Byrne, Jennifer Hoblyn

Trinity College, Dublin, Ireland (all authors)

Background: Huntington's disease (HD) is a rare autosomal dominant genetic disorder characterized by a triad of motor, psychiatric, and cognitive symptoms. Among psychiatric symptoms, depression is one of the common symptoms, which may appear several years prior to motor symptoms and correlates with suicide resulting in higher rates of premature death in HD. Although symptoms and signs of HD may appear distressing, it must be considered and remembered that depression and suicidal ideation are treatable conditions. It is suggested, however, that HD patients with depressive symptoms should also be treated with standard approaches. Comprehensive clinical guidelines are lacking due to the dearth of specific data for tailored approaches in HD. Novel approaches to the suppression of HD are promising, and a lot of effort has been focused on these potential new treatments. However, such developments do not negate the need to optimize the response to existing treatments. Clinicians must ensure that the drug treatment offered provides the optimal response for patients to alleviate the symptoms of HD.

Methods: Data were obtained from Enroll-HD for a total of 15,301 participants. A total of 3910 individuals were identified with depressive symptoms, and from these, 1300 were selected according to inclusion criteria for statistical analyses. SPSS Software version 26 will be used to perform mixed-model tests in order to examine the use of several different drug agents for ameliorating depression scores in those participants over a period of 4 years. Results for depression assessments and scores will be compared with control groups, untreated patients, and standard guidelines for depression in the general population.

Results: It is hoped that the statistical analyses will provide a clear picture of which antidepressive treatment is more successful in improving depression scores, particularly in patients with $\mathrm{HD}$, so as to evaluate whether this treatment can be considered a gold-standard for this condition.

\section{4}

Changes in Brain Activity with Antisense Oligonucleotide Tominersen Treatment in Early-Manifest Huntington's Disease

David Hawellek ${ }^{* 1}$, Pilar Garces ${ }^{1}$, Amir H. Meghdadi ${ }^{2}$, Shani Waninger ${ }^{3}$, Anne Smith ${ }^{4}$, Marianne Manchester ${ }^{5}$, Scott A. Schobel ${ }^{1}$, Joerg F. Hipp ${ }^{1}$

${ }^{1}$ F. Hoffmann-La Roche Ltd., Basel, Switzerland

${ }^{2}$ University of Manitoba, Winnipeg, Manitoba, Canada

${ }^{3}$ The Johns Hopkins University, Baltimore, MD, USA

${ }^{4}$ Ionis Pharmaceuticals, Inc., Carlsbad, CA, USA

${ }^{5}$ London Business School, London, UK

Background: Electroencephalography (EEG) measures neuronal activity directly and is known to be altered in Huntington's disease (HD). Previous analyses of the effects of tominersen (most recently known as RG6042), an antisense oligonucleotide targeting huntingtin premRNA/ 
mRNA, on a set of predefined EEG features in individuals with early manifest HD yielded negative results. The EEG data were reanalyzed with a data-driven approach that is capable of revealing effects not captured by the predefined features.

Methods: Forty-six individuals with early-manifest HD participated in a randomized, double-blind, multiple-ascending dose, placebo-controlled Phase I/IIa trial of tominersen. Resting-state EEG was recorded at baseline and at days 113, 141, and 197 after treatment, following the intrathecal injection of four monthly doses of tominersen.

Results: Compared to baseline, tominersen treatment resulted in an increase in EEG signal power within the 4-8-Hz frequency range, which was reduced in these patients at baseline when compared with healthy controls. The comparison to the placebo group revealed a significant difference between treatment groups, with a decrease of signal power for the placebo group within the same frequency range. This treatment effect was widespread across the scalp. The increase in brain activity with tominersen treatment was detectable across all dosing levels and was present throughout the duration of the posttreatment EEG monitoring. Conclusions: Tominersen mediated plastic changes to the EEG that opposed the HD EEG abnormalities and are, therefore, suggestive of a potential recovery of brain activity. However, other potential reasons for the change in EEG signal are possible and require further investigation. The relationship of these findings to therapeutic benefit awaits further investigation. EEG signal power at rest may be a critical functional biomarker in HD for monitoring disease progression and therapeutic effects.

\section{5}

\section{Epidemiology of Huntington's Disease in the US Medicare Population}

Alex Exuzides* ${ }^{1}$, Valerie Crowell ${ }^{2}$, Sheila Reiss Reddy ${ }^{3}$, Eunice Chang ${ }^{3}$, George Yohrling ${ }^{4}$

${ }^{1}$ Genentech, Inc., South San Francisco, CA, USA

${ }^{2}$ F. Hoffmann-La Roche Ltd., Basel, Switzerland

${ }^{3}$ Partnership for Health Analytic Research, LLC, San Francisco, CA, USA

${ }^{4}$ Huntington's Disease Society of America, New York, NY, USA

Introduction: Huntington's disease (HD) is a genetic, progressive, neurodegenerative disease with onset usually occurring between 30 and 50 years of age; however, late-onset HD can occur among older individuals. Few estimates of HD epidemiology in the USA exist; a recent study of commercially insured individuals calculated incidence and prevalence at 1.2 and 6.5 per 100,000 individuals, respectively. Given this dearth of evidence, particularly for older individuals, research is needed to generate current estimates in US populations. This study aims to estimate the current incidence and prevalence of HD among Medicare beneficiaries in the USA.

Methods: Medicare Research Identifiable Files (100\%) were used to identify beneficiaries $\geq 65$ years who were diagnosed with HD based on the presence of $\geq 1$ medical claim with a diagnosis for HD (International Classification of Diseases, 10th Revision, Clinical Modification: G10) in 2017. Prevalence was calculated as the number of HD cases in 2017 divided by all beneficiaries enrolled in Fee-for-Service Medicare and Part D in 2017 (reported per 100,000 persons). Incidence was calculated as the number of new HD cases in 2017 (i.e., no HD claim in 2016) divided by total at-risk patient-years from January 1 to diagnosis (cases) or enrolment end (non-cases) in 2017 (reported per 100,000 personyears). Estimates were stratified by sex.

Results: HD incidence among beneficiaries in 2017 was 6.1 per 100,000 person-years (females: 5.9 versus males: 6.4 ). HD prevalence in 2017 was 13.1 per 100,000 individuals (females: 12.6 versus males: 13.7 ).
Conclusions: This study of HD epidemiology among Medicare beneficiaries is the first of its kind. These estimates of incidence and prevalence are higher than previously reported in the USA; this may be due to diagnostic changes over time or differences in studied populations. Future estimates will be stratified by age and disease stage.

\section{6}

Clinical Characteristics of Late-Onset Huntington's Disease in North Americans from the Enroll-HD Study

Xiaoye Ma* ${ }^{1}$, Rita Gandhy ${ }^{2}$, Xiao-Yu Lu, Diana Slowiejko ${ }^{3}$, Samuel Frank $^{4}$

${ }^{1}$ Genentech, Inc., South San Francisco, CA, USA

${ }^{2}$ The Parkinson's Institute and Clinical Center, Sunnyvale, CA, USA

${ }^{3}$ Genentech, Inc., South San Francisco, CA, USA

${ }^{4}$ Beth Israel Deaconess Medical Center, Boston, MA, USA

Background: This study described demographics and clinical characteristics of individuals with late-onset Huntington's disease (LoHD) $(\geq$ 60 years of age at diagnosis) in North America.

Methods: Demographics, motor function, comorbidities, and longitudinal disease progression were summarized for North American participants in the Enroll-HD study with clinically manifest LoHD and a documented cytosine adenine guanine $(\mathrm{CAG})$ repeat size $(n=431)$. North American participants who were negative for the HD-causing mutation and $\geq 60$ years of age were included as controls $(n=361)$.

Results: The proportion of individuals with LoHD in North America was $19.2 \% ; 93.5 \%$ were Caucasian. Mean age at enrolment was 68.90 years and mean CAG repeat size was $40.89 ; 85.6 \%$ had CAG repeats between 40 and 42. The most common comorbidities in the LoHD group were psychiatric, cardiovascular, and musculoskeletal diseases, and incidences of several comorbidities, including psychiatric, ophthalmological, and neurological, were statistically higher in the LoHD group than in controls. The most common cancers reported in the LoHD group were skin, breast, and prostate. In individuals with $\geq 1$-year of follow-up in the LoHD group $(n=241)$, scores on the composite Unified HD Rating Scale (UHDRS) worsened by 0.84 points; a similar rate of worsening occurred in adultonset HD (0.82). Gait declined in $28.1 \%$, and walking ability declined in $17.5 \%$ of the LoHD group, as measured by UHDRS. Potential associations of genetic, clinical, and social characteristics, including CAG, use of concomitant medication, and presence of care provider, etc., along with disease progression in the LoHD, group were evaluated.

Conclusions: Prevalence of LoHD in Enroll-HD was higher than previously reported. Disease progression is complex in LoHD and correlates with multiple factors including genetic, social, motor, psychiatric, and cognitive.

Differential Burden of Comorbidities for Huntington's Disease, Parkinson's Disease, and the General Population for Patients and Care Partners in the USA

Alex Exuzides*1 ${ }^{1}$ Joana E. Matos, Bryan Ricker, Danny Bega ${ }^{2}$

${ }^{1}$ Genentech, Inc., South San Francisco, CA, USA

${ }^{2}$ Northwestern University, Evanston, IL, USA

Background: Huntington's disease (HD) is a rare, genetic neurodegenerative disease with cognitive, behavioral, and motor symptoms leading to increasing disability and loss of independence, impacting patients (PT) and care partners (CP). Parkinson's disease (PD) is also a 
neurodegenerative disease and, while HD is less prevalent with symptoms developing around 30 to 50 years, PD affects $1 \%$ of adults over the age of 60 . Age of onset and disease progression may be impacted by differential comorbidities. The objective of this study was to describe the comorbidity burden for PT and CP of $\mathrm{HD}, \mathrm{PD}$, and the general population (GP)

Methods: A cross-sectional online survey was administered in the USA, June-August 2019, to HD-PT/CP recruited through Rare Patient Voice. Recruitment was designed to be representative across HD stages. Self-reported physician diagnosis of anxiety (AX), bipolar disorder (BD), depression (DP), cancer (CA), diabetes (DB), and heart disease were compared to PD-PT $(n=118)$ and CP $(n=385)$, and GP $(n=123)$ and CP $(n=240)$ matched on age and gender from the 2018 US National Health and Wellness Survey. HD-PT/CP physical, mental, and emotional health are reported. Bivariate analyses were used for: (1) HD-PT and GP and (2) HD-CP and GP-CP. Multivariate analyses were used for: (1) HD-PT and PD-PT, controlling for age and gender, and (2) HD-CP and PD-CP, controlling for gender.

Results: Forty-one HD-PT and $80 \mathrm{HD}-\mathrm{CP}$ (not linked) were identified. $\mathrm{AX}$ was more common in HD-PT than GP $(p=0.001)$ and PD-PT $(p=$ 0.009 ), and in HD-CP than GP-CP and PD-CP (both $p<0.001$ ). BD was higher in HD-PT than GP $(p=0.045)$ and higher in HD-CP than GP-CP $(p=0.014)$. DP was higher in HD-PT than GP $(p=0.002)$ and higher in HD-CP than GP-CP and PD-CP (both $p<0.001$ ). CA was lower in HDPT than PD-PT ( $p=0.019)$, and lower in HD-CP than GP-CP $(p=0.021)$ and PD-CP $(p=0.012)$. DB was lower in HD-CP than PD-CP $(p<0.001)$. Poor/fair physical health was reported by $46.3 \%$ of HD-PT and $21.3 \%$ of HD-CP; $58.5 \%$ of HD-PT and $16.3 \%$ of HD-CP reported poor/fair mental health; $46.3 \%$ of HD-PT and $36.3 \%$ of HD-CP reported poor/fair emotional health.

Conclusions: Mental health comorbidities were most common among HD-PT/CP. The majority of HD-PT reported poor/fair mental health, while nearly half reported poor/fair emotional health. There is a need for ensuring provision of adequate mental health and preventive services to $\mathrm{HD}$ - and PD-PT/CP.

\section{8}

\section{Confidential Genetic Testing in a Non-nedical Setting: a Pilot Study}

Bonnie Hennig-Trestman*, Mary Edmondson, Debbi Fox-Davis, Katherine Sherry

\section{HD Reach, Raleigh, NC, USA (all authors)}

Background: Only 10-20\% of people at-risk for Huntington's disease (HD) undergo genetic testing. Psychological and social challenges are suggested factors that contribute to low testing utilization. Over a 10-year period, North Carolina-based HD Reach received nearly 300 inquiries related to at-risk/confidential testing. Typical medical and academic testing centers can be limited by an inability to offer confidential testing, lack of psychiatric services, and cost of private pay options. This pilot study seeks to examine the outcomes of a non-medical, confidential genetic testing program for individuals at-risk for HD.

Methods: A pilot program to address the need for confidential genetic testing was created. Goals of the program included reducing structural barriers to client engagement; focusing on individual choice, adjustment to risk, education, and preparation for genetic testing; expanding options for care; and exploring factors related to financial sustainability, replicability, and scalability of the testing program. The program highlights mental health services for at-risk individuals including bridge treatment during the decisionmaking and genetic-testing phases; facilitation of social supports for client/testing partner; psychoeducation directed at coping skills; confidential/anonymous genetic testing using a standardized HD Reach protocol; and telehealth technology to reach people regardless of location. Clients were screened for active mental health diagnoses, prior mental health history, and social determinants of health by a licensed social worker and a psychiatrist. If considered unstable (pre-existing or new DSM-V disorder, suicidality, active aggression, or inadequate coping skills) they were referred for mental health counseling. If they were stable or showed no evidence of mental health symptoms, they proceeded with genetic testing.

Results: A total of ten people at-risk for HD were recruited. Seven participants completed the program. Two were in the process of testing and one was completing enrollment. Genetic testing results included one confirmatory clinical test, one premanifest test, one asymptomatic gene carrier, three non-gene carriers, and one person declined testing due to pregnancy. Gene-positive participants were referred to medical professionals at centers with established HD programs. During follow up contact, participants described importance of confidentiality, decreased fear of unauthorized discloser of data, and flexibility of appointments.

\section{9}

\section{Longitudinal Trends of Clinical Outcome Measures in Patients with Pediatric Huntington's Disease}

Simona Rossomanno* ${ }^{1}$, Flavia Pizzagalli, Hannah Furby ${ }^{2}$, Dylan Trundell $^{1}$, Oliver Quarrell, Scott A. Schobel ${ }^{1}$

${ }^{1}$ F. Hoffmann-La Roche Ltd, Basel, Switzerland

${ }^{2}$ Roche Products Ltd, Welwyn Garden City, UK

Background: Enroll-HD (part of CHDI Foundation Project) is an ongoing multinational and multi-center observational study of Huntington's disease (HD). The registry was created to aid understanding of the dynamic phenotypic spectrum and the disease mechanisms of HD by collecting natural history data-covering the cognitive, behavioral, and motor symptoms of HD and associated impacts on daily function - and by collecting biological plasma samples. While this study mainly enrolls adult individuals with $\mathrm{HD}$, it is also open to younger individuals below the age of 18 years. Pediatric HD ( $<18$ years) differs from adult HD in that chorea is less frequently observed, and bradykinesic and dystonic symptoms typically occur at an earlier stage of disease. To date, investigation of clinical outcome measures has been limited to the Unified HD Rating Scale-Total Motor Score (UHDRS-TMS) in a juvenile HD population (onset $\leq 20$ years), and published data for the pediatric HD population, and for other clinical assessments, are currently lacking.

Methods: This study describes a retrospective analysis of longitudinal changes of clinical outcome measures in pediatric HD patients using Enroll-HD data (Periodic Dataset 4). For this selected patient population, the baseline characteristics and change from baseline over time of clinical outcome measures, such as UHDRS-TMS, were summarized.

Results: Twenty-five pediatric HD patients were registered in Enroll-HD and considered for the analysis. Baseline characteristics and change from baseline over time for the relevant clinical outcome measures will be presented.

Conclusions: These data provide insight into the longitudinal trends and reliability of clinical outcome measures collected in the Enroll-HD study. This will be relevant to understanding the natural history of HD in pediatric patients and for supporting drug development in a pediatric HD indication. 
20

Survival, Mortality, and Causes of Death in Huntington's Disease Patients in the UK

Hannah Furby*1, Loes Rutten-Jacobs ${ }^{2}$, Thanos Siadimas ${ }^{2}$, Filipe B. Rodrigues $^{3}$, Edward J. Wild ${ }^{3}$

${ }^{1}$ Roche Products Ltd, Welwyn Garden City, UK

${ }^{2}$ F. Hoffmann-La Roche Ltd, Basel, Switzerland

${ }^{3}$ University College London, London, UK

Background: Huntington's disease (HD) is a rare, genetic, neurodegenerative, and ultimately fatal disease, which can reduce life expectancy, often due to secondary causes such as pneumonia and other infections. This study characterized survival, mortality, and cause of death in HD in UK electronic medical records (EMR).

Methods: This study used UK Clinical Practice Research Datalink data, eligible for linkage to Hospital Episodes Statistics and Office for National Statistics databases. Incident cases aged $\geq 18$ years with first recorded HD diagnosis during the study period (January 2000 to December 2018) were included. Non-HD controls were matched 3:1 to HD cases based on birth year, gender, geographical region, and socioeconomic status (index of multiple deprivation) and had similar follow-up times. Kaplan-Meier methods were used to plot survival and log-rank-assessed differences in survival distributions. Differences in risk of death between groups were assessed using Cox proportional hazards models, with adjustment for potential confounders.

Results: Death occurred in 106/264 (40\%) of HD and 114/792 (14\%) of non-HD individuals during the study period. Median $(95 \%$ confidence interval [CI]) age of death in HD and non-HD cohorts was 71 (59-79) and 81 (73-86) years, respectively. Median survival time from first recorded HD diagnosis was 8.4 (95\% CI 6.7-9.8) years. Survival probability differed between groups $\left({ }^{2}=109.1, p<0.0001\right)$. Risk of death was 4.5 times higher in the HD group (hazard ratio $=4.50,95 \%$ CI 3.34-6.05; $p<0.0001)$. Common causes of death in the HD cohort were HD (60\%), cancers $(10 \%)$, dementias $(5 \%)$, myocardial infarction (3\%), and respiratory disorders $(3 \%)$.

Conclusions: This study demonstrates an increased risk of death in HD cases compared to non-HD cases using UK EMR data. HD was the most commonly reported underlying cause of death, which likely differs from the immediate cause of death.

\section{1}

Epidemiology of Huntington's Disease in the US Medicaid Population

Alex Exuzides* ${ }^{1}$, Sheila Reiss Reddy ${ }^{2}$, Eunice Chang ${ }^{2}$, Caleb Paydar ${ }^{2}$, George Yohrling ${ }^{3}$

${ }^{1}$ Genentech, Inc., South San Francisco, CA, USA

${ }^{2}$ Partnership for Health Analytic Research, LLC, San Francisco, CA, USA

${ }^{3}$ Huntington's Disease Society of America, New York, NY, USA

Background: Huntington's disease (HD) is a genetic, progressive, neurodegenerative disease characterized by cognitive, behavioral, and motor symptoms. Onset of HD usually occurs between 30 and 50 years of age, with an average survival of 15 years after diagnosis. Few estimates of HD epidemiology in the USA exist; a recent study using a commercial administrative claims database calculated prevalence at 6.5 per 100,000 persons. This study estimated HD prevalence among beneficiaries covered by Medicaid, a US federal-state public insurance program that provides healthcare coverage to low-income individuals.
Methods: Medicaid Analytic eXtract data from 17 states were used to identify beneficiaries $\leq 64$ years old who were diagnosed with $\mathrm{HD}$ based on the presence of $\geq 1$ medical claim with a diagnosis for HD (International Classification of Diseases, Ninth Revision, Clinical Modification code: 333.4 ) in 2014. Only non-dual eligibility with Medicaid and fee-for-service Medicaid beneficiaries were included in the study. Prevalence proportion was calculated as the number of HD cases in 2014 divided by all beneficiaries enrolled in 2014 (reported per 100,000 persons). Estimates were stratified by sex, age category $(\leq 17$, 18-34, 35-44, 45-54, 55-64), and disease stage (early, middle, late).

Results: In 2014, 353 Medicaid beneficiaries were identified as having $\mathrm{HD}$, translating to a prevalence of 15.2 per 100,000 persons (females: 19.6; males: 10.6). HD prevalence increased by age category $(1.1,12.3$, $50.6,65.1,83.7)$. Disease prevalence was highest in beneficiaries with late-stage $\mathrm{HD}(3.5,2.2,9.6)$.

Conclusions: This is the first study of HD epidemiology among Medicaid beneficiaries in the USA. These estimates of HD prevalence are higher than those previously reported in other US patient populations, which may reflect both the age of onset of HD and the unique eligibility qualifications of Medicaid resulting in a population with greater disability.

\section{2}

Neural Connectivity as a Substrate for Behavioral Compensation in Premanifest HD

Sarah Gregory* ${ }^{1}$, Peter A. Wijeratne ${ }^{1}$, Katherine Osborne-Crowley ${ }^{1}$, Eileanoir B. Johnson ${ }^{1}$, Paul Zeun ${ }^{1}$, Rachael I. Scahill ${ }^{1}$, Jessica Lowe ${ }^{1}$, Akshay Nair ${ }^{1}$, Claire O'Callaghan ${ }^{2}$, Christelle Langley ${ }^{2}$, Marina Papoutsi ${ }^{1}$, Carlos Estevez-Fraga ${ }^{1}$, Kate Fayer ${ }^{1}$, Daniel C. Alexander ${ }^{1}$, Trevor W. Robbins ${ }^{2}$, Barbara J. Sahakian ${ }^{2}$, Sarah J. Tabrizi ${ }^{1}$, Geraint Rees $^{1}$

${ }^{1}$ University College London, London, UK

${ }^{2}$ University of Cambridge, Cambridge, UK

Background: During the early stages of Huntington's disease (HD), behavioral performance is maintained in the presence of neuronal loss. We suggest that this is due to the onset of compensatory processes, where early striatal loss is accompanied by an absence of detectable cognitive deficits. We have previously developed and validated a model of compensation that identified increased functional brain connectivity as a potential substrate of compensation in HD gene-carriers 10 to 15 years prior to clinical diagnosis. In the current study, we have investigated compensation in a group of young adult HD gene-carriers (preHD), up to 24 years from disease onset, who display the earliest signs of pathology, but with no detectable behavioral deficits.

Methods: We used Dynamic Causal Modeling and Parametric Empirical Bayes to investigate differences in effective brain connectivity between age and sex-matched preHD and healthy control individuals in a series of key cognitive networks. Connections that differed between groups were then included in our compensation model and evidence for compensatory processes tested.

Results: There was a consistent pattern of higher connectivity from the striatum to the cortex in the preHD group compared to controls across most cognitive domain networks. We then identified evidence of compensatory processes for the spatial working memory network in four connections originating from the striatum.

Conclusions: Higher levels of connectivity from the striatum to the cortex in preHD suggest an upregulation of brain connectivity to counter the earliest effects of pathology. The very beginnings of the onset of compensation, focused on the spatial working memory network, is indicative of the way in which compensation may develop during preHD. 
23

Identifying Impediments to Healthcare Delivery for Huntington's Disease Patients During COVID-19

Anna Pfalzer*, Elizabeth Huitz, Danielle A. Buchanan, Brittany K. Brown, Sarah Moroz, Renee M. Rouleau, Kaitlyn R. Hay, Amy Laird, Abagail Ciriegio, Kelly H. Watson, Maile Jones, Ya-Chen Lin, Hakmook Kang, Heather Riordan, David A. Isaacs, Katherine McDonell, Bruce E. Compas, Daniel O. Claassen

Vanderbilt University Medical Center, Nashville, TN, USA (all authors)

Objective: We assessed the feasibility of telehealth, economic vulnerability, and stress of persons with Huntington's disease (HD) as a result of the COVID-19 pandemic.

Methods: This is a cross-sectional observational study of HD patients and their caregivers. Participants were contacted via phone by clinical staff and answered open-ended questions regarding the impact of COVID-19; economic hardship; and need for clinical resources, along with their ability to access to a local physician, medications, ability to do telehealth, and willingness to participate in an online support group. All participants completed a 10-question Likert-scale assessment of perceived stress. Lastly, patient demographics, medical insurance information, current access to an online health portal, and clinical information on disease burden were obtained from the medical record.

Results: During stay-at-home orders, the vast majority of HD patients are able to obtain medications, however, nearly $25 \%$ of patients and caregivers did not wish to commit to regular telehealth visits. Approximately $60 \%$ of participants indicated being adversely impacted by the COVID19 pandemic, with those from lower-income counties noting a substantive economic impact. Patients and caregivers noted elevated stress, with patient disease burden being negatively correlated with patient stress.

Conclusions: The sudden and dramatic shift toward telemedicine necessitated by the COVID-19 pandemic has provided detailed insight into limitations of our current healthcare infrastructure. Furthermore, we find that families are particularly vulnerable to the economic and psychological effects of stay-at-home orders. These observations illustrate areas for clinical care improvement to address economic and healthcare disparities in our communities.

24

Using Spatial Memory to Bridge the Translational Gap Between Animal and Human Cognitive Assessment for Huntington's Disease Clinical Trials

Yifat Glikmann-Johnston*, Emily-Clare Mercieca, Anna M. Carmichael, Bonnie Alexander, Ian H. Harding, Julie C. Stout

Monash University, Melbourne, Australia (all authors)

Background: Cognitive impairment in Huntington's disease (HD) is a key symptom that devastates patients and for which no treatments exist. Clinical drug trials that target cognition in HD are developed on the basis of treatment successes in animal studies. These successes, however, have failed to translate to humans. We have recently shown a disconnect between cognitive testing methodologies in animals and humans in HD trials, thus minimizing the likelihood that what is observed in animal models will be translated to humans. To bridge this gap, we suggest to focus on spatial memory in HD because it is one of the few cognitive domains that can use highly comparable behavioral testing methods across species. In order to determine the suitability of spatial memory for cognitive assessment in HD clinical trials, we examined the relationship between spatial memory and the brain pathology of HD.
Methods: We studied 25 peri-manifest HD individuals, comprising 12 participants up to 10 years to predicted clinical diagnosis (pHD) and 13 participants with early manifest HD (mHD), and a comparison group of 32 matched healthy controls. We examined spatial memory using the Paired Associates Learning task from the Cambridge Neuropsychological Test Automated Battery and an experimental Virtual House task. Volumes of the caudate nucleus, putamen, and hippocampus were manually segmented using T1-weighted MRI.

Results: Spatial memory differed significantly between peri-manifest HD and controls (all $p<0.002$ ), with mHD performing worse than $\mathrm{pHD}$ and controls. As expected, caudate nucleus and putamen volumes were smaller in the peri-manifest HD group compared to controls (both $p<0.001$ ), but hippocampal volumes did not differ significantly between groups $(p=0.4)$. Within the peri-manifest HD group, caudate nucleus (all $p<0.009$ ), putamen (all $p<0.01$ ), and hippocampal $(p<0.03)$ volumes were associated with spatial memory performance, whereby smaller volumes were related to worse spatial memory.

Conclusions: Our findings link spatial memory impairment to caudate nucleus, putamen, and hippocampal volumes in peri-manifest HD, suggesting that the time is ripe to include spatial memory in the cognitive phenotype of HD. For HD clinical trials, although there are many reasons why preclinical successes in animals do not translate to clinical trials in humans, our results suggest that it would be beneficial to consider aligning cognitive testing across species in HD clinical trials.

\section{5}

Small Ubiquitin-Like Modifier Promotes the Mammalian Target of Rapamycin Kinase Signaling and Pathogenesis in Huntington's Disease

Uri Ramírez-Jarquín*, Neelam Shahani, Manish Sharma, Srinivasa Subramaniam

The Scripps Research Institute Jupiter, FL, USA (all authors)

Background: Small ubiquitin-like modifier (SUMO) is a posttranslational modification that alters protein-protein interactions, localization, and stability of proteins, but its role in the regulation of the mammalian target of rapamycin (mTOR) kinase, which is aberrantly upregulated in neurological and neurodegenerative disorders, remains unknown.

Results: Here, we report that SUMO promotes mTOR signaling and the pathogenesis of Huntington's disease (HD), a genetic disorder caused by polyglutamine expansion of huntingtin (mHTT) that affects the striatum and other brain regions. Depletion of SUMO (1, 2, and 3) impairs the amino acids (AA)-sensitive mTORC1 signaling (as measured by $\mathrm{pS} 6 \mathrm{~K} / \mathrm{pS} 6$ ), as well as AA-insensitive mTORC2 signaling (pAkt S473) in the HD striatal neuronal cells. SUMO depletion diminishes the mTOR and raptor (mTORC1 component) and mTOR and rictor (mTORC2 complex) interactions but does not interfere with the mTOR and GbL interaction. SUMO 1 deletion in HD knock-in mice (SUMO1-/-; q175HDhet) robustly prevented HD-associated motor deficits compared to HD mice (q175HD-het). Furthermore, in a newly developed calorie-restriction mouse model injected with AA via retroorbital (CAAR) model, we found that AA-induced mTORC1 activation in the striatum of $\mathrm{q} 175 \mathrm{HD}$-het is abrogated in the SUMO1-/-; q175HD-het mice.

Conclusions: These data indicate that SUMO regulates mTOR signaling in the HD brain and regulates age-associated HD pathogenesis. Targeting the SUMO pathway may halt and/or ameliorate HD pathogenesis by blocking nutrient-induced aberrant mTORC1 signaling. 
26

Speech is a Sensitive Marker in Premanifest Huntington's Disease: Acoustic Features and Their Relationship to Cognition and FineMotor Performance

\author{
Jess Chan ${ }^{*}$, Yenni Lie ${ }^{2}$, Julie Stout ${ }^{2}$, Adam Vogel ${ }^{1}$ \\ ${ }^{1}$ The University of Melbourne, Melbourne, Australia \\ ${ }^{2}$ Monash University, Melbourne, Australia
}

Background: Subtle motor and cognitive changes can manifest prior to diagnosis in Huntington's disease. Speech disturbance is a common but underutilized clinical feature of premanifest HD (PreHD). Acoustic analysis offers objective data on cognitive-linguistic behavior presenting in HD.

Objective: To explore the sensitivity of acoustic analysis for detecting changes in speech function prior to diagnosis in HD. A secondary aim was to examine the impact of cognitive and fine-motor functioning on speech.

Methods: Speech data were acquired from 55 people with the expanded HD gene (14 presymptomatic individuals, PresymHD; 18 prodromal individuals, ProdromHD; 14 early-stage HD; 9 mid-stage HD) and 55 ageand sex-matched healthy controls. Subjects completed the following tasks: syllable repetition, sustained vowel, and automatic and connected speech tasks. Data were analyzed acoustically for measures of articulatory agility, voice quality, and speech timing. The Cogstate Brief Battery and the Purdue Pegboard Test were also used to examine cognition and finemotor performance.

Results: Speech timing differed between PresymHD (far from disease onset) and matched controls on connected speech tasks only, which is the most cognitively demanding task in the protocol. Speech fluency in reading tasks was interrupted and the rate of speech was slower, with longer silences in people with PresymHD. A similar speech pattern was observed between people with ProdromHD (within 15 years to estimated disease onset) and their matched healthy controls. Speech in ProdromHD is characterized by reduced speech agility, slower rate of speech, prolonged pause times between phrases, and higher proportion of silences. A decline in speech agility correlated significantly with reduced fine-motor performance.

Conclusions: Subtle, but apparently genuine, differences were observed in the ProdromHD and PresymHD groups compared to controls. These differences were greater than those observed on the cognitive tests. Future work is needed on the stability and reliability of acoustic metrics over time, along with the relationship of these deficits with underlying neuropathology.

\section{7}

\section{A Randomized Controlled Trial of the Efficacy of a Probiotic Intervention to Modulate Gut Microbiota and Symptoms in Huntington's Disease}

Cory Wasser* ${ }^{1}$, Emily-Clare Mercieca ${ }^{1}$, Geraldine Kong ${ }^{2}$, Anthony J. Hannan $^{2}$, Sonja McKeown ${ }^{1}$, Yifat Glikmann-Johnston ${ }^{1}$, Julie C. Stout ${ }^{1}$

${ }^{1}$ Monash University, Melbourne, Australia

${ }^{2}$ Florey Institute of Neuroscience and Mental Health, Victoria, Australia

Background: People with Huntington's disease (HD) experience a range of gastrointestinal symptoms and unintended weight-loss. Despite increasing evidence that probiotics improve gut function, mood, and cognition, this is yet to be explored in HD. We sought to extend our previous findings of gut dysbiosis in HD and conducted a randomized controlled clinical trial investigating the effect of a probiotic supplement
(Lactobacillus rhamnosus, Saccharomyces boulardii, and Bifidobacterium) on the gut microbiome, cognition, and clinical outcomes. Our primary outcome was to determine whether a sixweek probiotic intervention significantly improved gut microbiome composition in HD gene expansion carriers (HDGECs) and healthy controls (HCs). We further explored whether probiotics improved cognition, and self-reported depression and gastrointestinal symptoms.

Methods: We compared 41 HDGECs, including 19 people with manifest HD (Total Functional Capacity $>6$ ) and 22 premanifest HDGECs, with 36 age and gender-matched HCs. We obtained informed consent; administered cognitive tests and questionnaires about gut and depression symptoms; and obtained fecal samples and then analyzed using 16S V3-V4 rRNA sequencing.

Results: In our previous study (Wasser et al., 2020), and in this study, at both baseline and postprobiotic intervention, HDGECs had lower alpha diversity (species richness and evenness) and altered beta diversity (microbiome structure) compared to $\mathrm{HCs}$, indicating stability of gut microbiome differences between groups across time. Nevertheless, at the microbiome level, we did not find changes after probiotic intervention in species richness and evenness (alpha diversity), nor in microbiome structure (beta diversity) in HDGECs, HCs, or across the whole sample. Furthermore, alpha diversity showed good within-subjects reliability (average intraclass correlation coefficient of .776, $p<0.001$ ). For our exploratory outcomes, probiotic intervention did not significantly improve performance on cognitive tasks, nor on self-reported depressive or gastrointestinal symptoms.

Conclusions: Although our probiotic intervention was not beneficial in this instance, we showed a stable difference between HDGECs and HCs in the gut microbiome. Given clinical evidence of gut dysfunction and cognitive impairment in HD, and our recent findings of gut dysbiosis at the microbiome level, the gut presents as a promising target for future therapeutic interventions aimed at delaying disease onset and slowing progression.

\section{8}

\section{Navigating the Challenges of COVID-19: Staying Active with HD}

Una Jones*, Monica Busse

Cardiff University, Cardiff, Wales, UK (all authors)

Background: Current clinical recommendations suggest that people with Huntington's disease (HD) take part in moderate aerobic exercise paired with strengthening exercises three times per week to improve and maintain motor function. Recent social distancing restrictions due to COVID19 have created further challenges for those seeking regular physical activity and for those providing support. A new approach to supporting people with HD to start and stay active was urgently needed. We, therefore, developed mechanisms that would allow people with HD to access existing resources to support them to remain active during social distancing.

Methods: Through discussion with charity organizations, e.g., Huntington's Disease Association of England and Wales (HDA) and the Physiotherapy Working Group of the European Huntington's Disease Association (EHDN), existing resources were reviewed, translated if necessary, and made available to people with HD. A webinar was developed and transmitted in April 2020, and summaries of resources were disseminated via the EHDN.

Results: The webinar "Keeping Physically Active During Coronavirus" was well received by people with $\mathrm{HD}$, their caregivers, and health and exercise professionals from the UK, USA, and Europe. The Move to Exercise booklet that accompanies YouTube videos was shared with English-speaking organizations in Australia and translated into Italian, 
Spanish, and Portuguese for dissemination on HD organization websites. PAT-HD, a tool to support people in developing a personalized physical activity plan, was disseminated via the HDA website.

Conclusions: In a relatively short time, it was possible to coordinate, translate, and disseminate resources to support people with HD to keep active during a pandemic. People who used the resources found them helpful and informative, and supported in developing a personalized physical activity plan. With people becoming more used to remote ways of communicating during COVID-19, there is now an opportunity to further develop ways to support people with HD using telehealth that could incorporate multidisciplinary healthcare teams that involve the HD family within their own community.

\section{9}

\section{Acceptability of a Co-designed Tool to Promote Physical Activity in People with Huntington's Disease}

\author{
Una Jones*, Katy Hamana, Monica Busse \\ Cardiff University, Cardiff, Wales, UK (all authors)
}

Background: The movement problems of chorea, bradykinesia, and dystonia associated with Huntington's disease (HD) are compounded by slowing of information processing, depression, and apathy, and lead to decreasing functional independence. Despite growing evidence, there remains a lack of uptake of exercise and/or physical activities in people with HD, an issue often compounded by difficulty accessing support from healthcare professionals. The aim of this study was to evaluate the acceptability of a co-designed physical activity support tool for use within a HD clinic. Co-design was essential to ensure that the tool met the needs of the HD community.

Methods: A prototype tool was developed through review of literature and a visual-led methodology. The prototype was used in a workshop with people with HD, their caregivers, and healthcare professionals to co-design a physical activity tool for HD (PATHD). Activities included discussion on appropriate physical activities for people with HD, how such a tool could be used, and feedback on the prototype tool. PAT-HD was co-designed as a two-sided paper-based tool to promote and support individualized planning of physical activity. PAT-HD was piloted for 9 months in a HD specialist clinic in England, UK. We recorded details of physical activity discussions in the clinic and conducted semistructured interviews with three clinic staff members to evaluate acceptability of PAT-HD.

Results: Forty people visited the HD clinic during the study period; 19 were given physical activity advice. From the interviews, themes around who (People), where (Setting), and how (Approach) promotion of physical activity could take place were identified; concepts of benefits and barriers were threads through each theme. The theme of People included both people with HD and the healthcare professionals providing support; Setting included appropriateness of HD-specific clinics or communities for supporting people; and Approach included the need for individualized support.

Conclusions: PAT-HD is a simple paper-based tool that can be used to support conversations about physical activity with people with HD and to develop a personalized physical activity plan. Barriers to developing the plan included time and lack of knowledge of local resources by the healthcare professional. Further work in exploring the use of PAT-HD within community settings and as a digital tool, particularly during social distancing, is required.

\section{0}

Evaluation of Blood Glial Fibrillary Acidic Protein as a Potential Marker in Huntington's Disease

Tengteng $\mathrm{Wu}^{1}$, Yixuan Zeng ${ }^{2}$, Lishan Lin, Dingbang Chen, Chao Wu, Huihua Yang, Xunhua L., Zhong Pei*2

${ }^{1}$ Beijing Institute of Technology, Beijing, China

${ }^{2}$ The First Affiliated Hospital, Sun Yat-sen University, China

Background: Huntington's disease (HD) is an autosomal dominant neurodegenerative disorder. Neurofilament light protein (NfL) is correlated with clinical severity of HD participants, but relative data are lacking in the Chinese population. Reactive astrocytes are related to HD pathology, which predicts their potential to be a biomarker in HD progression. The role of blood GFAP to evaluate clinical severity when performing novel disease-modifying interventions needs to be discussed.

Methods: Twelve healthy controls and $46 \mathrm{HD}$ participants were recruited in this study. Demographic data and clinical severity were assessed with the international Unified Huntington's Disease Rating Scale (UHDRS) and retrospectively analyzed. Plasma NfL and GFAP were quantified with an ultra-sensitive, single-molecule (Simoa) technology. We explored the consistency of these two biomarkers and their correlation with clinical severity.

Results: Both plasma NfL and GFAP were increased in Chinese HD mutation carriers and they were linearly correlated with each other $(r=$ $0.563, p<0.01)$. The difference in concentration between stage 1 and 2 in manifest HD participants was slightly larger in plasma GFAP $(p<0.01)$ compared with NfL $(p<0.05)$. These two analytes were also significantly correlated with disease burden, Total Motor Score (TMS), and Total Functional Capacity (TFC). The scores of stroop word reading and symbol digit modalities tests were correlated with plasma NfL but not with plasma GFAP.

Conclusions: Plasma GFAP was correlated with plasma NfL and clinical severity in HD participants. Thus, plasma GFAP may have the potential to be a sensitive biomarker for evaluating disease progression when performing disease-modifying therapies in manifest HD participants.

31

Examining the Effect of Exercise on the Progression and Severity of Huntington's Disease Using Different Covariate Balancing Methods and Simulated Data Derived from the PACE-HD Study

Andreas Markoulidakis, Katie Taiyari, Peter Holmans, Philip Pallmann, Monica Busse, Beth Ann Griffin*

Cardiff University, Cardiff, Wales, UK (all authors)

Background: Regular activity and structured exercise are hypothesized to lessen the impact of Huntington's disease (HD). Randomized controlled trials (RCTs) are the gold standard for measuring the causal effects of both pharmacological and non-pharmacological interventions on clinical outcomes. RCTs are, however, challenging to conduct and in certain situations, causal intervention effects may be inferred from observational studies. Observational study designs do not allow conclusions about causal relationships to be drawn unless statistical techniques are used to account for the imbalance of confounders that occur in the groups being compared. Propensity score (PS) and balance weighting are useful techniques that can be used to reduce the imbalances between groups by weighting the groups to look alike on the observed confounders. There are many methods available to estimate PS and balancing weights. 
However, it is unclear a priori which will achieve the best trade-off between balance and power. Weighted analyses are further complicated by small sample sizes, common in HD.

Methods: We provide step-by-step guidance on how to estimate causal effects using observational study data for HD studies and compare the performance of a number of commonly used estimation methods on simulated data derived from the Physical Activity and Exercise Outcomes in HD (PACE-HD) study, which is designed to explore whether enhanced physical activity affects the progression and severity of HD and reflects many of the realworld challenges for evaluating exercise interventions in HD. Using data from 26 individuals receiving enhanced physical activity and 58 continuing as usual, we aimed to balance the groups on key pretreatment confounders measured at intake that capture baseline severity of HD and fitness levels.

Results: We present a comparison of the balance that each method achieved based on different balance metrics (e.g., standardized mean differences and Kolmogorov-Smirnov statistics), as well as details on the power of each method. Our work shows clear preference for use of entropy balancing weights in our application.

Conclusions: We provide general guidelines for the choice of method for estimation of PS and balancing weights, interpretation of results, and careful assessment of assumptions such as overlap and unobserved confounding bias. We underscore how the lessons can be applied more broadly to other HD studies using observational data with small sample sizes.

\section{2}

\section{Cognitive Dysfunction and Decline in Juvenile-Onset Huntington's} Disease

Hend Al-Kaylani*1, Kathleen E. Langbehn ${ }^{1}$, Ellen van der Plas ${ }^{1}$, Mark D. Bowren, Eric Epping, Peggy Nopoulos ${ }^{1}$

${ }^{1}$ University of Iowa, Iowa City, IA, USA

Background: Assessment of cognitive dysfunction and rate of decline may help clinicians determine Juvenile-Onset Huntington's Disease (JOHD) prognosis and design support strategies. This study aimed to identify group differences in baseline cognitive performance and decline between JOHD participants and those who are at-risk (from a family with HD), but tested Gene NonExpanded (GNE).

Methods: The analysis included 54 observations from 24 JOHD individuals and 203 observations from 115 GNE individuals. JOHD participants had molecular confirmation of CAG expansion and a clinical diagnosis determined using the Unified Huntington's Disease Rating Scale (cut-off score of 15). JOHD subjects were early in the course of their disease with a mean disease duration at time of initial assessment of 2.35 years $(\mathrm{SD}=3.88)$. Participants completed the adult (WAIS) or childhood (WISC) Wechsler intelligence scales to measure general cognitive ability, and the Delis-Kaplan Executive Function System (DKEFS) to measure executive function. We conducted mixed linear regression models to compare the groups at baseline, as well as comparison of change over time.

Results: At baseline, the JOHD group scored $\sim 2$ SD lower than the GNE group mean on all indices of the WISC/WAIS and D-KEFS. The groupby-elapsed-time interaction was significant for all WISC/WAIS variables (all $p<0.001$ ) and the Letter Fluency, Category Fluency, Category Switching, and Word Reading D-KEFS variables (all $p<0.05$ ). The JOHD group exhibited significant decline in points/year, while the GNE group exhibited little to no change in scores. On the general ability index (GAI), the JOHD group declined 6 points on average (2 SD decline in scores over five years); GNE increased 0.2 points. On Category Fluency, the JOHD group decreased 2.1 points while the GNE group declined 0.1 points.

Conclusions: Significant cognitive deficits were evident at baseline in individuals with JOHD relative to GNE which is remarkable given the fact that most were seen soon after diagnosis. This could be due to lack of full ascertainment of cognitive skills (in those that develop the disease very young) or significant decline in function prior to motor onset. In addition, the JOHD group declined rapidly over a period of $\sim 4$ years. Quantifying the rate of cognitive dysfunction may help validate cognitive screening tools to assist in recognition and prognosis of JOHD non-motor symptoms, and predict other cognitive and behavioral symptoms prevalent in HD.

\section{3}

\section{Investigating the Therapeutic Potential of Antibodies Targeting Phosphorylated Tau in HD Mice}

Maria Masnata*1, Helena Denis, Martine Saint-Pierre, Peter Davies ${ }^{2}$, Emmanuel Plane ${ }^{1}$, Francesca Cicchetti ${ }^{1}$

${ }^{1}$ Université Laval Québec City, Quebec, Canada

${ }^{2}$ Feinsteing Institutes for Medical Research

Background: Tau is a microtubule-associated protein that is enriched in axons, where it is thought to regulate microtubule dynamics, neurite outgrowth, transport, and synaptic plasticity. Abnormally hyperphosphorylated tau may accumulate in the form of neurofibrillary tangles (NFTs) and/or neuropil threads (NTs), two entities that interfere with these fundamental functions. This has been classically associated with Alzheimer's disease; however, tau pathology has been postulated to be a common feature of many neurodegenerative conditions, including Huntington's disease (HD). Here, we hypothesize that targeting tau in vivo improves HD features, both at the behavioral and cellular levels.

Methods: We administered the antitau pS202 CP13 monoclonal antibody to early symptomatic zQ175 mouse model of HD. The mice received $10 \mathrm{mg} / \mathrm{kg}$ weekly intraperitoneal injections of CP13 antibody for a total of 12 weeks. To evaluate motor and cognitive performance, open field and Y-maze tests were performed, once before the start of treatment followed by monthly testing. The nesting test was performed at the end of the treatment to evaluate the animal's overall well-being. At the completion of the study, mice were sacrificed, and their brains collected for postmortem analysis.

Results: After 3 months of treatment, zQ175 mice demonstrated improved motor performance (increased distance traveled), cognition (increased long-term and short-term memory, along with improved working memory), and general health, while anxiety-like behavior, typical of zQ175 mice, was not altered by CP13 injection. Phosphorylated tau (pS202 and pS199) was reduced in the cortex and hippocampus of HD treated animals, but was unaltered in the striatum, a structure that is not primarily showing signs of tauopathies associated with HD. Furthermore, we detected increased levels of vesicular glutamate transporter 1 (VGLUT1) in both the cortex and hippocampus in zQ175, suggesting that CP13 improved synaptic functionality. Preliminary observations indicate that CP13 treatment can impact mutant huntingtin (mHTT) aggregation.

Conclusions: These preliminary findings suggest that reducing phosphorylated tau improves motor and cognitive deficits and ameliorates hallmarks of HD pathology, such as synaptic dysfunction and mHTT aggregation, in an HD mouse model. 
34

Does the Blood-Brain Barrier Contribute to Pathological Protein Spread in Huntington's Disease?

Melanie Alpaugh ${ }^{* 1}$, Giacomo Sciacca ${ }^{2}$, Luc Bousset ${ }^{3}$, Ronald Melki ${ }^{3}$, Francesca Cicchetti ${ }^{1}$

${ }^{1}$ Université Laval, Quebec City, Quebec, Canada

${ }^{2}$ Université du Quebec, Quebec, Canada

${ }^{3}$ Loyola University, Chicago, IL, Canada

Introduction: Studies of neurodegenerative conditions have demonstrated that disease-associated pathological proteins can behave in a prion-like manner, contributing to disease manifestation and/or progression. In Huntington's disease (HD), a number of studies have shown that similar mechanisms apply to mutant huntingtin (mHTT), including evidence of spread from the circulatory system to the brain (Rieux et al., 2020), suggesting that mHTT can cross the blood-brain barrier (BBB). In addition to crossing the $\mathrm{BBB}$, several reports have indicated that mHTT is present within endothelial cells and that HD patients have vascular abnormalities, including decreased integrity of the BBB, changes in the expression of tight-junction proteins, and an increase in vessel density. Given that several of these disease features were replicated in wild-type mice after exposure to mHTT in the blood stream (Rieux et al., 2020), we aim to test whether the presence of mHTT in the circulation contributes to these changes and to further understand how this may participate to the spread/clearance of mHTT. Our hypothesis is that mHTT can cross the BBB, and that the presence of pathological mHTT surrounding the neurovascular unit contributes to diminished BBB integrity and to changes in vascular morphology.

Methods: We developed a 3D human BBB model containing control or HD patient-iPSC-derived endothelial cells (dBMEC) and astrocytes, as well as primary brain pericytes. This model was subsequently treated with $\mathrm{N}$-terminal HTT protein fragments containing either 19 or 48 polyglutamine repeats.

Results: Both patient and control-dBMEC form leak-tight barriers after $96 \mathrm{~h}$ in culture. When these vessels are treated with mHTT and WT HTT for $48 \mathrm{~h}$, HTT is found to enter endothelial cells and cross the BBB in control and HD patient-derived cells. While both forms of HTT show the capacity to cross the BBB, they have different effects on the permeability of the vessel with mHTT, causing a greater detrimental change to barrier integrity than WT HTT when added to the lumen of the vessel. When HTT is added to the compartment containing glia, the effects on the barrier are magnified but occur in response to both WT and mHTT.

Conclusions: Using a human cell culture model of the BBB, we observed bi-directional crossing of HTT in a CAG tract size independent manner. Additionally, the presence of fibrils impacts the integrity of the BBB suggesting that mHTT itself may contribute to vascular abnormalities previously reported in HD patients, as well as to the spread/clearance of mHTT.

References: Rieux, M., et al., 2020. Shedding a new light on Huntington's disease: how blood can both propagate and ameliorate disease pathology. Mol Psychiatry.

35

Using the Roche Huntington's Disease Digital Monitoring Platform in a 15-Month Clinical Trial: Analysis of Participant Satisfaction

Atieh Bamdadian*1, Cedric Simillion ${ }^{2}$, Florian Lipsmeier ${ }^{1}$, Anne Smith ${ }^{3}$, Edward J. Wild ${ }^{4}$, Scott A. Schobel ${ }^{1}$, Michael Lindemann ${ }^{1}$
${ }^{1}$ F. Hoffmann-La Roche Ltd., Basel, Switzerland, Switzerland

${ }^{2}$ Universitat Bern, Bern, Switzerland

${ }^{3}$ Ionis Pharmaceuticals, Inc., Carlsbad, CA, USA

${ }^{4}$ University College London, London, UK

Introduction: Continuous remote monitoring of Huntington's disease (HD) signs and symptoms has the potential to complement standard inclinic tests that may be limited by inter-rater variability, subjectivity, and naturally occurring fluctuations in symptom intensity. The Roche HD digital monitoring platform (dMP) consists of smartphone-based active tests, as well as smartphone- and smartwatch-based passive monitoring of participants during daily activities. Preliminary data from the 15-month open-label extension (OLE) of the phase I/IIa study (NCT03342053) of tominersen (most recently known as RG6042) in patients with early manifest HD has shown that remote monitoring using the dMP yields reliable and valid measures of cognition and motor symptoms. It is important to assess the level of satisfaction with the dMP from a participant's perspective, as this will impact adherence and could therefore have a subsequent effect on the utility of dMP data in clinical studies and patient care. This study investigated participant satisfaction with the dMP using data from the OLE study.

Methods: All 46 OLE study participants were requested to answer a questionnaire (10 questions in total) at their last in-clinic visit. The questionnaire was designed to assess overall participant satisfaction with the $\mathrm{dMP}$ and to highlight its strengths and weaknesses in terms of usability. Results: Thirty-three of 46 participants completed the questionnaire. Of all responders, $82 \%$ felt satisfied or neutral about the dMP overall, and 97\% felt satisfied or neutral about the instruction from site staff and supporting information they received. Over $70 \%$ of responders found that the length and process of performing their daily active tests was acceptable. Of all participants, $80 \%$ felt positive or neutral about collecting smartwatch passive monitoring data, whereas $70 \%$ were positive or neutral about smartphone-collected passive data. Approximately $70 \%$ of patients found it easy or very easy to plan time for active tests every day. Around 25\% of patients found wearing the smartwatch difficult, and $17 \%$ would prefer not to use the smartwatch as a component of the dMP at all. Similar numbers were found for passive monitoring with the smartphone. Conclusions: Overall, most participants were satisfied with the dMP and performing active tests on a daily basis. The majority of participants were satisfied with using the dMP for passive monitoring, with the smartwatch preferred over the smartphone for this purpose.

\section{6}

Using Artificial Intelligence for Fast, Reliable, and Automatic Segmentation of the Thalamus

Michael Reinwald*1, Eileanoir B. Johnson ${ }^{1}$, Rachael I. Scahill ${ }^{1}$, Robin Wolz $^{2}$

${ }^{1}$ IXICO PLC and University College London, London, UK

${ }^{2}$ Imperial College London, London, UK

Background: Changes in volume of the thalamus are used as biomarkers to track the development of Huntington's disease (HD) and to monitor the potential effects of interventional treatments; therefore, accurate volume calculations, obtained via segmentations, are of utmost clinical importance. These regions are particularly challenging for both manual and automated methodologies, due to unclear region boundaries. We present a fully automated approach that works reliably across different datasets and outperforms popular analysis tools.

Methods: We developed a deep neural network (NN) for the task of semantic binary segmentation of the thalamus in 3D T1-weighted MRI images (T1). The architecture of the network features an analysis path, using T1s as an input, and a synthesis path, with the thalamus 
segmentation as an output. We trained the $\mathrm{NN}$ on $30 \mathrm{~T} 1 \mathrm{~s}$ from the OASIS dataset and their corresponding thalamus segmentations, which have been manually edited by an academic expert with 8 years of experience in subcortical segmentation. We validate the network on various open-source datasets (i.e., ADNI, HCP, OASIS), as well as in-house HD clinical trial data. We assess segmentation quality manually and compare our results against other widely used analysis tools, such as Freesurfer (FS) and volbrain (VB).

Results: Our results show superior segmentation quality of the NN throughout all datasets. The FS segmentations had rough boundaries, and were often too large, with the thalamus region-of-interest (ROI) spilling into neighboring regions in both anterior and posterior directions. FS also had examples whereby the ROI was excluding posterior regions of the thalamus. VB segmentations show better consistency; however, they suffer from tight boundaries between the thalamus and the CSF, resulting in underestimation of the thalamus. NN segmentations are the most consistent, with smooth boundaries that overlaid the thalamus well. Furthermore, the segmentation can be processed in seconds, which is several orders of magnitude faster than FS or VB.

Conclusions: Our NN shows very good generalization capabilities on unseen datasets, encouraging the application on new HD populations to obtain superior segmentation results within a fraction of the time of other analysis tools. We demonstrate that our method provides rapid, reliable segmentation-based thalamic volumes across a variety of datasets, suggesting it has feasibility for implementation in large clinical trials in HD.

\section{7}

\section{Investigation of the Humoral Immune Response Against Huntingtin} Via the Development and Validation of an ELISA Assay

Helena L. Denis*1 ${ }^{1}$, Melanie Alpaugh ${ }^{2}$, Claudia Alvarez, Martine SaintPierre $^{1}$, Luc Bousset ${ }^{3}$, Ronald Melki ${ }^{4}$, Rachel J. Harding ${ }^{5}$, Francesca Cicchetti $^{1}$

${ }^{1}$ Université Laval, Québec, Canada

${ }^{2}$ Université du Quebec, Quebec, Canada

${ }^{3}$ Loyola University, Chicago, IL, Canada

${ }^{4}$ French National Center for Scientific Research, Paris, France

${ }^{5}$ University of Toronto, Toronto, Canada

Background: A well-described aspect of Huntington's disease (HD) is the activation of immune cells throughout the body, which may be particularly relevant to the pathology since they can contribute to the clearance of misfolded pathologic proteins. As demonstrated in Alzheimer's disease (AD), antibodies raised against amyloid beta, the signature misfolded protein of this disease, are significantly increased in the plasma of $\mathrm{AD}$ subjects with mild cognitive impairments when compared to agematched controls. To date, the mechanisms of clearance of mutant huntingtin (mHTT) protein aggregates in HD have not been fully elucidated. We explored the possibility that the immune system recognizes mHTT as a pathogen and, consequently, develops an antibody-response specific to the mutated protein.

Methods: To assess this, we developed an ELISA assay to quantify the presence of antibodies against various forms of $\mathrm{MHTT}$ in plasma derived from 1) WT mice injected intravenously with fibrils containing either 25 (HTT) or 48 (mHTT) polyglutamine $(\mathrm{Q})$ repeats (1 injection every 2 weeks for 3 months), 2) HD mouse model, the zQ175 knock-in model, and 3) human HD samples from premanifest to late-stage disease according to the clinical evaluation of the Unified Huntington's Disease Rating Scale (UHDRS). To validate this ELISA assay, we first used a commercial antibody to ensure the specificity of the signal obtained. We then proceeded with the extraction and purification of antibodies from the plasma by immunoprecipitation with commercial protein $\mathrm{A} / \mathrm{G}$ beads and by using an exclusion/concentration Amicon-column with a cut-off at $100 \mathrm{kDa}$. We focused our study on the identification of the immune response against the fibrillar forms of HTT (Q25) or mHTT (Q48) and the full-length HTT protein with either Q23 (HTT) or Q54 (mHTT form).

Results: Peripheral administration of Q25 and Q48 fibrils to WT mice resulted in a strong antibody response against these fibrillar forms of HTT/mHTT. After purification and concentration of antibodies raised in these animals, only mice injected with Q48 fibrils developed antibodies, which also recognized the full length mHTT (Q54) antibodies. Ongoing experiments will unveil whether plasma from HD mouse models or patients contain similar antibodies.

Conclusions: Our findings suggest that the humoral immune response can generate antibodies against mHTT. If such antibodies are found in patients, this would shed light on a novel aspect of the pathology.

\section{8}

Normative Curves for Selected Unified Huntington Disease Rating Scale Measures

James Mills ${ }^{1}$, Jeffrey D. Long* ${ }^{2}$, Amrita Mohan ${ }^{3}$, Jennifer J. Ware ${ }^{3}$, Cristina Sampaio $^{3}$

${ }^{1}$ Wave Life Sciences, Cambridge, MA, USA

${ }^{2}$ University of Iowa, Iowa City, IA, USA

${ }^{3}$ CHDI Management, Inc., New York, NY, USA

Objective: To examine the effects of natural aging for those without Huntington's disease (HD) gene expansion and to develop normative curves by age, sex, and education from the distribution of observed scores for the Symbol Digit Modalities Test (SDMT), Stroop Word Reading Test (SWRT), Total Motor Score (TMS), and Total Functional Capacity (TFC) from the UHDRS along with their composite, known as the composite UHDRS (cUHDRS).

Background: The progression of HD for gene-expanded carriers (HDGEC) is well-studied. Natural aging effects, however, are not often considered in the evaluation of HD progression.

Methods: After combining longitudinal REGISTRY and Enroll-HD data from the most recent periodic dataset (PDS4 from Enroll-HD), we fit natural cubic splines with a knot at the median age using linear quantile mixed models for participants without HD gene expansion $(N=3731, N$ observations $=9451$ ). Normative curves were estimated for the 0.05 , 0.50 , and 0.95 quantiles. Two types of reference curves were considered; unconditional curves were only dependent on age while conditional curves were dependent on age and other covariates (e.g., education and/ or sex).

Results: Conditioning on education was necessary for SDMT, SWRT, and cUHDRS. Unconditional curves were sufficient for the TMS. TFC was unique in that the curve was constant over age with its intercept being at the maximum score $(\mathrm{TFC}=13)$. For all measures, sex effects were minimal so conditioning on sex was unnecessary.

Conclusions: Extreme quantile estimates for each measure (SDMT, SWRT, TMS, and cUHDRS) can be considered as boundaries for natural aging and HDGEC scores falling beyond these thresholds are likely the result of HD progression. Normative curves and tables are developed that can serve as references for clinical characterization of HDGEC.

39

Instrumentation of Assessments in Huntington's Disease

Jo Shattuck*, Lauren Seeberger

University of Colorado, Aurora, CO, USA (all authors) 
Background: Measuring motor behavior is usually conducted with visual assessments with subjective scores assigned on a numeric scale $0-5$ to assess specific movements. The goal of this study was to improve quality and reliability of the measurement of motor symptoms in a clinical setting by adding motion-capture instruments during the movements. This study was modeled after a comprehensive motor-assessment study conducted by Dr. Lori Quinn in 2019 at Columbia Teacher's College in which wearable motion-capture sensors (APDM) were correlated with the UHDRS motor score and other quantitative measurements in a full motion-capture lab. That study conducted a comprehensive 90-min assessment in a laboratory setting, among other home-based activity tracking. Those results suggested the APDM measurements of sway, chorea, and jerk offered the most promise for use in an in-clinic assessment. Our purpose was to test the feasibility of a shorter 20-min version assessment in a clinical setting with the APDM system.

Methods: In contrast to 90-min lab-setting assessments, we ran a subset of the full battery at our monthly HD clinics that included various balance/sway measures, sit to stand, hand flip, and gait. All subjects $(n=11)$ gave their consent to the study in accordance with the informed consent regulations of CU Anschutz. The data collected during the 20min assessment for the measures involved six APDMs. APDMs worn by the patient during motor assessments were compared to a UHRDS taken within 3 months.

Results: The APDM built-in assessments ( $\mathrm{z}$ scores) were used in Pearson's product moment correlations for three $Z$-score normalized, APDM measures: Jerk, Sway Area, Sway Root Mean Squared (RMS) with the UHDRS (motor sub score), TFC, and CAP Scores. (No familywise adjustments/corrections were used for multiple correlations.) The correlations of UHDRS Total Motor score to all three APDM measures were stronger than the TFC and CAP Scores: UHDRS correlation with Sway Area, Sway RMS, and Jerk were $r=0.78$ and $p=0.005, r=0.79$ and $p=0.003$, and $r=0.78$ and $p=0.005$, respectively, $n=11$.

Conclusions: Preliminary analysis on UHDRS and Jerk measures showed the highest correlation with manual measures. More subjects and the data analysis on chorea measures are needed. However, early results suggest the abbreviated APDM test shows similar correlations to the full battery conducted by Dr. Quinn. Continued trends in this study offer support for using APDM sensors for HD assessments and could improve diagnostic confidence and the test-retest reliability and reduce the subjectivity of UHRDS-related assessment variability in clinical settings.

\section{0}

SEEING-HD: Social and Economic Evaluation of Individual and Generational Burden of HD

\section{Colin Green}

\section{Shift Health, Toronto, Ontario, Canada}

Background: The overall burden of Huntington's disease (HD) has been relatively under-studied. Available evidence does not appear to characterize key aspects of the burden of HD (e.g., quality of life, economic impact), including elucidating how this burden is shared among patients, families, and caregivers; how this burden evolves as the disease progresses; and how disease burden impacts generations. The lack of robust evidence characterizing the complex, multifactorial nature of HD limits our ability to make evidence-based decisions about care that can improve the lives of patients, families, and caregivers. To this end, SEEING-HD is a novel, patient-informed study aiming to address these evidence gaps and create a more holistic understanding of the burden of manifest HD.

Methods: We have assembled a global team, including HD experts and patient groups from Denmark, Germany, France, Italy, Poland, Spain, and the United Kingdom to guide our study and ensure that our approach and outputs reflect leading thinking in the field and the voices of patients, families and caregivers. We will begin by conducting a systematic literature review and an assessment of data collected by existing patient registries and other studies. We will then close evidence gaps through primary research involving patients, their families, and caregivers across the seven countries to collect information on measures of quality of life (e.g., health, education), socioeconomic burden (e.g., direct and informal care costs), and wider societal impacts.

Results: SEEING-HD is a large-scale study that, to our knowledge, is the first to take a comprehensive approach to exploring the intergenerational burden of manifest HD. Informed by patients, their families, and caregivers, this study will generate evidence that will allow us to characterize the impact of the disease on quality of life by stage and by stakeholder affected by HD (e.g., the individual, parent, child, caregiver) and inform the development of a conceptual model demonstrating the intergenerational impact of HD. The complete methodology will be presented at the conference.

Conclusions: By examining the burden of HD from a multidimensional, generational, patient-informed perspective, our study represents a significant contribution to the knowledge base that will empower stakeholders to advance evidence-based initiatives to improve the lives of people impacted by HD.

\section{1}

\section{Early Olfactory Memory Deficits in HD and Unawareness of} Olfactory Dysfunction

Fabricio Joao Pio* ${ }^{1}$, M. Lessard-Beaudoin ${ }^{2}$, E. Peachey, Devine Calanog $^{1}$, Terri Petkau ${ }^{1}$, G. Leonard, M.A. Pouladi, B.R. Leavitt ${ }^{1}$, R.K. Graham $^{2}$

${ }^{1}$ University of British Columbia, Vancouver, British Columbia, Canada ${ }^{2}$ University of Sherbrooke, Sherbrooke, Canada

Background: Olfactory dysfunction is a feature of several neurodegenerative disorders, including Huntington's disease (HD). These deficits occur early and correlate with a decline in global cognitive performance, depression, and structural abnormalities of the olfactory system. Limited studies have assessed olfactory memory in HD, and whether HD individuals are aware of their olfactory dysfunction.

Methods: The UPSIT and ODMT were used for the olfactory testing in control $(n=6)$, premanifest $(n=8)$, and manifest HD individuals $(n=6)$. The age range was $30-59$ years. The UPSIT entails the recognition of 10 odors correctly from 40 choices. The ODMT is a non-verbal screen of both odor discrimination and memory. The olfactory self-report includes: Do you have problems with the sense of smell? Response options: "No," "Yes," "I don't know." UBC CREB ethics approval \#H18-01586 and all subjects gave their consent.

Results: A decrease was observed in UPSIT scores when comparing HD individuals with controls (Kruskal-Wallis $p<0.01$, Dunn post hoc Control vs. HD $p=0.011$ ). Furthermore, there was a significant decrease in the ODMT total test scores revealing that deficits in odor memory are present early in HD (Kruskal-Wallis $p<0.0001$, Dunn post hoc Control vs. HD $p=0.006$ ). In both the UPSIT and the ODMT, a significant linear trend was observed (UPSIT $p=0.0007, r=0.8825$; ODMT $p=<0.0001$, $r=0.9756$ ). In addition, a deficit was observed in each of the ODMT delay time points for HD individuals, and predominantly in the 30 - and 60-s delay intervals for the preHD (2-way RM ANOVA, Interaction $p=$ 0.54 , Time $p=0.89$, Genotype $p<0.0001$, Subjects $p=0.16$, Turkey post hoc Control vs. preHD $p=0.003$; Control vs. HD $p<0.0001$; preHD vs. $\mathrm{HD} p=0.001$ ). There was also a difference in the frequency of the subcategories of olfaction between the three groups with both the preHD and HD individuals showing increases in the affected categories (Fisher's exact test $p=0.046$ ). Differences between the groups were also observed 
when comparing the self-report and the UPSIT of those who responded "no" (Fisher's exact test $p=0.01$ ) and in the number of individuals in the three groups correctly identifying their level of olfaction (Fisher's exact test $p=0.01$ ).

Conclusions: We show that deficits in odor identification, discrimination, and memory occur in preHD and HD individuals. Of note, the majority of HD individuals are unaware of their olfactory dysfunction. Unawareness of olfactory problems poses a risk for the individual and may lead to malnutrition and depression.

42

\section{Palliative Care in Huntington's Disease: a Systematic Review}

Jocelyn Jiao $^{1}$, Amie Hiller*2

${ }^{1}$ Keck School of Medicine, University of Southern California, CA, USA ${ }^{2}$ Oregon Health \& Science University, Portland, OR, USA

Background: Palliative care (PC) is defined by the World Health Organization as "an approach that improves the quality of life of patients and their families facing the problem associated with life-threatening illness, through the prevention and relief of suffering by means of early identification and impeccable assessment and treatment of pain and other problems, physical, psychosocial and spiritual." Though PC originated in oncology to manage patients struggling with cancer and treatment-related side effects, it has developed a burgeoning presence within neurology. Our goal in this publication is to systematically review the palliative literature as it relates to persons with Huntington's disease (HD).

Methods: We completed a Pubmed search, including the terms palliative care and Huntington's disease, and received 51 citations. After review of the titles and abstracts (and full articles if necessary), we were limited to 34 publications that are further detailed in our results section. An OVID search did not reveal any unique publications except for one non-English item, which was not included.

Results: Out of the 34 manuscripts, nine were primarily focused on HD care with brief mention of PC and 10 primarily focused on PC with brief mention of $\mathrm{HD}$; the remaining 15 manuscripts focused upon specific areas of palliative care in HD. In review of these, manuscripts largely fell within one of four categories: quality of life, late-stage care, juvenile HD, or end-of-life issues. Quality of life was the largest proportion at $40 \%$ $(6 / 10)$, then late-stage care at $4 \%$, juvenile HD at $3 \%$, and end-of-life issues at $2 \%$. Several articles noted areas of future research that require exploring to define and optimize PC in HD. We also included literature discovered via other search criteria that addressed pertinent issues, such as end-of-life wishes and multidisciplinary care models in HD.

Conclusions: Palliative care within the neurological field is in its relative infancy. The available literature that is specifically categorized as palliative care offered insights into quality-of-life issues, late-stage care, juvenile HD, and end-of-life care. A significant proportion of palliative HD literature is not necessarily categorized as such but still contributes to the interdisciplinary space between PC and HD.

43

\section{Criminal Behavior in Huntington's Disease}

Brittany Brown*, Katherine McDonell, Lisa Hale, Bruce E. Compas, Daniel O. Claassen

Vanderbilt University Medical Center, Nashville, TN, USA (all authors)

Background: An increased incidence of criminal behavior has been reported in Huntington's disease (HD) as compared with the general population (Jensen et al., 1998), although the overall prevalence of these behaviors in the HD population remains unknown.

Methods: We performed a retrospective chart review of 287 patients treated for HD at Vanderbilt University Medical Center from 2006 to 2020 to assess the prevalence and types of criminal behavior (defined as any illegal activity that may or may not have resulted in arrest) in our HD population. Clinical and demographic information were obtained from electronic medical records.

Results: We identified $32 \mathrm{HD}$ patients with criminal behaviors documented in their medical records, comprising $11 \%$ of the 287 patients reviewed. Of these patients, $65 \%$ were male. Mean CAG repeat length was 48 . Average age at the time of offense was 35, ranging from 17 to 72 . The most prevalent issue was physical violence, reported in 16 out of 32 patients (50\%). Other behaviors included reckless driving (38\%), substance abuse (25\%), inappropriate sexual behavior (13\%), and illegal financial activity (13\%). Out of the 32 patients, $19(59 \%)$ had been arrested, with seven having been arrested more than once for violence, substance abuse, and/or sexual misconduct. Comorbid psychiatric symptoms were reported in $100 \%$ of patients, with the most common being irritability $(81 \%)$, depression $(78 \%)$, and anxiety $(69 \%)$. Less frequent symptoms included perseveration $(56 \%)$, apathy $(50 \%)$, delusions (13\%), and hallucinations (3\%).

Conclusions: Our results emphasize that criminal behavior is common in HD, particularly in young adult males with comorbid psychiatric symptoms. These findings likely represent an underestimate of the true prevalence of these behaviors in our clinic population, given that this analysis was retrospective and limited to information documented in the medical record. There are likely several mediating factors contributing to criminal behavior in the HD population, including high rates of neuropsychiatric symptoms (Thompson, 2012) and significant psychosocial stressors (Downing, 2012; Mand, 2015), as well as impaired inhibitory control (Rao, 2014) and a tendency toward risk-taking (van Wouwe, 2016). Rigorous clinical screening for these activities and targeted interventions to address and prevent criminal behavior will be paramount in reducing the burden of these challenging issues in HD families.

\section{References:}

- Downing N, Smith MM, Beglinger LJ, et al. Perceived stress in prodromal Huntington disease. Psychol Health. 2012;27(2):196-209.

- Jensen P, Fenger K, Bolwig TG, Sorensen SA. Crime in Huntington's disease: a study of registered offences among patients, relatives, and controls. J Neurol Neurosurg Psychiatry. 1998;65(4):467-471.

- Mand CM, Gillam L, Duncan RE, Delatycki MB. "I'm scared of being like mum": The Experience of Adolescents Living in Families with Huntington Disease. J Huntingtons Dis. 2015;4(3):209-217.

- van Wouwe NC, Kanoff KE, Claassen DO, et al. The Allure of HighRisk Rewards in Huntington's disease. J Int Neuropsychol Soc. 2016;22(4):426-435

\section{4}

\section{GLUT1 and GLUT3 in Huntington's Disease Brain and Fibroblasts}

Maria Pia Abruzzese*1, Andrea Arena ${ }^{2}$, Sergio Fanelli ${ }^{2}$, Jessica Rosati ${ }^{3}$, Andrea Ilari $^{2}$, Jean-Paul Vonsattel ${ }^{4}$, Marzia Pierluigi ${ }^{2}$, Fernando Squitieri ${ }^{1}$

${ }^{1}$ Fondazione IRCCS Casa Sollievo della Sofferenza Research Hospital, San Giovanni Rotondo, Italy

${ }^{2}$ Sapienza University of Rome, Rome, Italy

${ }^{3}$ St. Joseph's College New York, Brooklyn, NY, USA

${ }^{4}$ Columbia University, New York, NY, USA

Background: The Huntington's disease (HD) pathological process is characterized by abnormal glucose and energetic metabolism in brain and peripheral tissues. We investigated the role of the two most 
representative glucose transporters, GLUT1 (i.e., mainly expressed in astrocytes) and GLUT3 (i.e., mainly expressed in neurons), in the brain cortex and in peripheral cell lines of HD patients.

Methods: Postmortem brain tissue (cortex) from eight adult patients (mutation length range: 43-57 CAG repeats) with different pathological grades of HD (from grades 2 to 4 ) and three age-matched healthy controls (HC) were examined in this study. Samples were obtained by the New York Brain Bank at Columbia University, New York, USA. Fibroblast cell lines were obtained by skin biopsies of early phase HD patients and age-matched $\mathrm{HC}$ subjects. The two juvenile-onset (joHD) patients carried a mutation length of 64 and 85 CAG repeats; the two adult patients (aHD) had a mutation length range of 42 and $43 \mathrm{CAG}$ repeats. Proteins were extracted for Western blot analysis, which was performed by specific GLUT1 and GLUT3 antibodies.

Results: The brain cortex expression of GLUT1 was similar for neuropathological HD grade-2 and HC. The expression level of GLUT1 for grades 3 and 4 was about $50 \%$ lower in the brain cortex than in HC. The GLUT3 expression level was similar in the brain cortex across all neuropathological HD grades to HC. Western Blot (WB) analysis on fibroblast cell lines from aHD, joHD, and $\mathrm{HC}$ subjects was performed on cell growth in culture medium without glucose for $24 \mathrm{~h}$ and then added after $24 \mathrm{~h}$ with $0 \mathrm{~g} / \mathrm{l}, 1 \mathrm{~g} / \mathrm{l}, 4 \mathrm{~g} / \mathrm{l}$ of glucose. The experiments showed a remarkable GLUT1 decrease in joHD cell lines.

Conclusions: GLUT1 deficiency contributes to the glucose hypometabolism and to the abnormal energetic metabolism in advanced HD. The reduced GLUT1 level in peripheral cell lines from joHD, but not from aHD, suggests a more extended pathological process that may likely contribute to the severe disease progression in patients with large-sized mutations.

\section{5}

Correlations Between Cognitive Scores and Behavioral Abnormalities to Detect New Potential Clinical Markers in Manifest Huntington's Disease

Sabrina Maffi*1 ${ }^{1}$, Simone Migliore, Giulia D’Aurizio, Consuelo Ceccarelli, Mariateresa Moscato ${ }^{2}$, Giovanni Ristori, Silvia Romano, Anna Castaldo ${ }^{3}$, Caterina Mariotti ${ }^{3}$, Giuseppe Curcio ${ }^{4}$, Fernando Squitieri ${ }^{1}$

${ }^{1}$ Fondazione IRCCS Casa Sollievo della Sofferenza Research Hospital, San Giovanni Rotondo, Italy

${ }^{2}$ University of Bologna, Bologna, Italy

${ }^{3}$ Fondazione I.R.C.C.S. Istituto Neurologico Carlo Besta

${ }^{4}$ Università degli Studi dell'Aquila, L'Aquila, Italy

Background: Huntington's disease (HD) behavioral changes start at the presymptomatic life stage with unpredictable progression. We investigated the possible association(s) between the severity of cognitive and behavioral symptoms in manifest HD.

Methods: We analyzed the longitudinal clinical data of 97 patients from three ENROLL-HD Centers and examined patients' motor, functional, and cognitive changes by the Unified Huntington's Disease Rating Scale (UHDRS) and behavioral changes by the Problem Behaviors Assessment Short Form (PBA-s) at three different yearly times of patients' lives ( $\mathrm{t} 0$ or baseline, $\mathrm{t} 1$ after 1 year, $\mathrm{t} 2$ after 2 years).

Results: The severity of apathy and perseveration/obsession symptoms scores were associated with the severity of the cognitive executive dysfunctions $(p<0.0001)$, regardless of the time-point, thus suggesting a significant correlation between cognition decline and the alteration of some specific neuropsychiatric patterns.

Conclusions: Although the severity of behavioral manifestations do not show a predictable progression in HD, the severity of apathy and perseveration/obsessions are significantly associated with the severity of the executive function impairment, thus contributing, together, to the progression of HD and to the patients' loss of independence, in addition to the neurological manifestations. This cognitive-behavior pattern determines a common underlying deficit attributable to a dysexecutive syndrome and may offer new clues to detect clinical markers in a still unexplored field of manifest HD.

\section{6}

Investigating the Contribution of Tau to Huntington's Disease Pathology

Shireen Salem*1 ${ }^{1}$, Melanie Alpaugh ${ }^{1}$, Luc Bousset ${ }^{2}$, Aurélie de Rus Jacquet $^{1}$, Ronald Melki ${ }^{2}$, Francesca Cicchetti ${ }^{1}$

${ }^{1}$ Université Laval, Québec, QC, Canada

${ }^{2}$ Loyola University, Chicago, IL, Canada

Background: The tau protein, implicated in microtubule stabilization and axonal transport in physiological conditions, is present in toxic state in several neurodegenerative disorders including in Huntington's disease (HD). HD is more specifically considered a $4 \mathrm{R}$ tauopathy, i.e., that the $4 \mathrm{R}$ tau isoform expression is increased by a splicing defect. Here, we hypothesize that the introduction of either $3 \mathrm{R}$ or $4 \mathrm{R}$ tau to cellular and animal models of HD leads to an exacerbation of intracellular mutant huntingtin (mHTT) aggregation, with consequences for cellular and behavioral functions.

Methods: Two models have been selected: (1) An HD neuronal cell line, StHdh cells containing 7 (StHdhQ7/Q7) or 111 (StHdhQ111/Q111) polyglutamine repeats and (2) a human neuroblastoma cell line, SHSY5Y cells which will be chemically transfected with CMV-plasmids carrying exon 1 of the human huntingtin gene. Human synthetic recombinant tau (monomers and fibrils of 1 N3R and 1N4R forms of tau) are introduced to the cells and various aspects of toxicity and dysfunction are assessed. The uptake of tau is observed by immunocytochemistry and metabolic activity using an MTT toxicity assay. HTT aggregation is measured by filter retardation assays and cell viability by investigating the apoptotic pathways, notably caspase levels and activity. To evaluate the influence of tau on cognitive impairments, bilateral intracerebral stereotaxic injections of tau will also be performed into two brain regions, the hippocampus and prefrontal cortex, of wild-type and HD (zQ175) mice at 3 months of age. Behavior tests will be performed to assess cognitive, motor, and anxiety-like behavior of mice.

Results: Preliminary experiments show that low dose (1 nM) 3R and $4 \mathrm{R}$ tau fibrils can significantly decrease the metabolic activity of both healthy and StHdhQ111/111 cells. Uptake of all forms of tau has been observed within cells, and the $3 \mathrm{R}$ forms of tau significantly increased HTT aggregation. Thus far, only baseline measures ( 2 months of age) have been collected to reveal no behavioral differences between groups.

Conclusions: Tau seems to be altering cellular aspects of HD, notably decreasing metabolic activities and increasing HTT aggregation within cells. Ongoing experiments will allow us to link the alterations observed at the cellular level to the behavioral aspects observed in HD mice models, shedding light on the role of tau in this disease.

47

Imaging and Clinical Data on Swallowing Function of Individuals with Huntington's Disease and Dysphagia

Megan Keage*¹, Shira Baum ${ }^{2}$, Lisa Pointon ${ }^{3}$, Jane Lau ${ }^{1}$, Jacinta Berndt ${ }^{1}$, Josephine Hopkins ${ }^{1}$, Roxanne Maule ${ }^{2}$, Adam P. Vogel ${ }^{1}$ 
${ }^{1}$ The University of Melbourne, Melbourne, Australia

${ }^{2}$ Herzog Hospital, Jerusalem, Italy

${ }^{3}$ Calvary Health Care Bethlehem, Victoria, Australia

Background: Dysphagia is common in Huntington's disease (HD) affecting all phases of swallowing. Correlations exist between noninstrumental measures of dysphagia and clinical features of HD, including age, disease duration, and degree of motor impairment. Lack of instrumental data limits our ability to wholly characterize HD-related dysphagia and prognosticate swallowing changes over time.

Objective: To retrospectively describe a relatively large database of videofluoroscopic studies (VFSSs) and determine the relationships between dysphagia and HD clinical parameters, including disease duration and burden of pathology score.

Methods: Medical and swallowing data of 49 individuals with HD and dysphagia were examined. VFSS data were interpreted using the Bethlehem Assessment Scale and Penetration-Aspiration Scale. Data from clinical bedside examinations and social information were collated to describe the impact of dysphagia in HD. Repeated VFSS data were available for seven individuals.

Results: Swallowing was characterized by lingual dysfunction, reduced soft palate elevation, delayed pharyngeal swallow initiation, and inability to clear matter from the pharynx. Two-thirds of cases presented with compromised airway protection with both liquid and solid consistencies. Tachyphagia and difficulty with self-feeding were common. Dysphagia correlated with disease severity and duration. Longitudinal analysis revealed a mixed pattern of progression with some individuals presenting with worsening dysphagia while others appeared to remain stable or improved in function.

Conclusions: Dysphagia in HD is exacerbated by difficulties with selffeeding and monitoring feeding rate. Burden of pathology relates to pharyngeal swallow initiation and penetration and aspiration of fluid. Dysphagia did not appear to worsen in a systematic way in a subset of participants.

\section{8}

\section{Enroll-HD Study Status}

Selene Capodarca ${ }^{*}$, Enroll-HD platform team

CHDI Foundation, Inc., New York, NY, USA

Enroll-HD is a clinical research platform that includes at its core an observational, prospective study of HD. The objectives of Enroll-HD are 1) enhance the design and expedite the conduct of clinical trials, 2) improve the understanding of the phenotypic spectrum and disease mechanisms, and 3) foster good clinical care. Over 23,800 participants (more than 19,750 currently active) have been recruited from 20 countries and 173 study sites (158 currently active). The data collected from these participants is monitored using a rigorous risk-based process. Recoded data and biosamples are made available to researchers. As of June 22, approximately 250 projects were conducted, and more than 50 publications were published using the Enroll-HD data. Enroll-HD also serves as a registry that can be used to facilitate recruitment by identifying potentially eligible participants who can be invited by investigators to participate in clinical trials. To improve support for future clinical trials and observational studies, the study, since the end of 2017 has been focusing on the recruitment of at-risk, premanifest, and early-stage participants. The implementation of this recruitment strategy is positively impacting the participant population, with an increase in the desired cohorts. During the second quarter of 2020 , the study has been greatly impacted by COVID-19, with a sharp decrease in the number of study visits. During the lockdown, the study team encouraged the sites to keep the study participants engaged in the study and collect some data through phone contacts. Since May, a gradual reopening of the sites was observed (as of 22 June 2020, 70 sites out of 157 have resumed study activities). Platform studies are clinical studies that utilize at least one or more types of EnrollHD platform support. These include site feasibility, study guidance documents and templates, potentially eligible participant listings, study setup support, monitoring, and data management. The Enroll-HD Clinical Training Portal is an online resource launched in January 2017, where HD research personnel can complete and maintain study-relevant training, presently UHDRS Motor Certification, GCP (all users), and Enroll-HD Plasma Collection (Enroll-HD study users only). There are currently more than 1850 active users, and it enables faster, more cost-effective start-up of clinical trials and studies, standardizes the quality of training, and reduces workload at sites.

\section{9}

\section{Untargeted Metabolomics and Pathway Analysis on Greek-Cypriot HD Patients}

Christiana Christodoulou*, Christiana A. Demetriou, Yiolanda Christou, George M. Spyrou, Eleni Zamba Papanicolaou

The Cyprus Institute of Neurology and Genetics, Nicosia, Cyprus (all authors)

Background: Huntington's disease (HD) is a rare, inherited autosomal dominant neurodegenerative disease affecting the medium spiny neurons of the basal ganglia. Exposure to the Mediterranean Diet (MD), which is high in antioxidants, provides neuroprotection, assisting the body's innate antioxidant system to eliminate ROS species. Furthermore, the MD has anti-inflammatory properties that decrease inflammatory cytokines and cells of the immune system from attacking self-cells. Metabolites are small compounds and they are intermediate end-products of metabolism. Metabolomics is the systematic study of metabolites from a biological sample (urine, serum, plasma, cerebral spinal fluid). Metabolomics are proving to be useful in the study of diseases as they are not only closer to the phenotype but also can provide possible biomarkers for disease. The Ingenuity Pathway Analysis (IPA) by Qiagen, was used to perform the metabolomics analysis to identify the pathways which become dysregulated in each HD stage.

Objectives: 1) To obtain a metabolomic profile of human serum samples using untargeted MS-based approaches in order to identify metabolites and their associated pathways that significantly differentiate presymptomatic from early manifest and advanced manifest patients with HD and therefore might be involved in phenoconversion and disease severity. 2) To obtain metabolomic profiles of human serum samples using untargeted MS-based approaches in order to identify metabolites and their associated mechanisms. 3) To examine whether the mechanisms and metabolites that are perturbed in different disease stages.

Methods: This is a case-control study consisting of presymptomatic $(n=$ $20)$, early symptomatic $(n=10)$, and advanced-stage $(n=8)$ HD patients, as well as age-matched controls $(n=37)$. Data regarding demographics, clinical characteristics, and lifestyle were collected. Untargeted metabolomics analysis was performed using Liquid chromatography-Mass spectrometry (LC-MS) approaches on serum samples obtained from Cypriot HD patients and healthy controls. Interpretation of the metabolomics data was performed using different bioinformatics tools and databases to identify metabolites and their associated biological pathways which become dysregulated in the different HD stages.

Results: The results of this study are expected to demonstrate an overlap between the pathways/metabolites perturbed in HD. The pathways and metabolites we identified such as the urea cycle, androgen biosynthesis, histamine degradation, and several additional pathways may provide us with a better understanding of how the metabolites within these pathways 
become dysregulated. This may possibly lead to therapeutic treatments that target the abnormal metabolite concentration and pathways.

Conclusions: The pathways we identified in our study may result in possible therapeutic treatments that target the abnormal metabolite concentration and pathways. This may lead to overall improvement in the quality of life of HD patients. A further investigation into the enzymes, metabolites, and substrates that become altered in these pathways can provide further understanding of how pathway dysregulation leads to HD.

\section{0}

Imbalanced Basal Ganglia Connectivity is Associated with Motor Deficits and Apathy in Huntington's Disease: First Evidence from Human In Vivo Neuroimaging

\author{
Akshay Nair \\ University College London, London, England, UK
}

Background: The gating of movement in humans is thought to depend on activity within the cortico-striato-thalamic loops. Within these loops, emerging from the cells of the striatum, run two opponent pathways: the direct and indirect pathway. Both are complex and polysynaptic but the overall effect of activity within these pathways is to encourage and inhibit movement, respectively. In Huntington's disease (HD), the preferential early loss of striatal neurons forming the indirect pathway is thought to lead to disinhibition that gives rise to the characteristic motor features of the condition. But early HD is also specifically associated with apathy, a failure to engage in goal-directed movement. We hypothesized that in $\mathrm{HD}$, motor signs and apathy may be selectively correlated with indirect and direct pathway dysfunction, respectively.

Methods: Using a novel technique for estimating dynamic effective connectivity of the basal ganglia, we tested both of these hypotheses in vivo for the first time in a large cohort of patients with prodromal HD $(n=94)$. We used spectral dynamic casual modeling of resting state fMRI data to model effective connectivity in a model of these cortico-striatal pathways. We used an advanced approach at the group level by combining Parametric Empirical Bayes and Bayesian Model Reduction procedure to generate a large number of competing models and compare them by using Bayesian model comparison.

Results: With this fully Bayesian approach, associations between clinical measures and connectivity parameters emerge de novo from the data. We found very strong evidence (posterior probability $>0.99$ ) to support both of our hypotheses. Firstly, more severe motor signs in HD were associated with altered connectivity in the indirect pathway and by comparison, loss of goal-direct behavior or apathy, was associated with changes in the direct pathway component of our model.

Conclusions: The empirical evidence we provide here is the first in vivo demonstration that imbalanced basal ganglia connectivity may play an important role in the pathogenesis of some of commonest and disabling features of HD and may have important implications for therapeutics.

\section{1}

Enhancing the Clinical Utility of DriveSafe DriveAware for People with Huntington's Disease

\author{
Monique Sondhu* ${ }^{1}$, Fiona Fisher, Julie C. Stout ${ }^{1}$ \\ ${ }^{1}$ Turner Institute for Brain and Mental Health, Monash University, \\ Clayton, Melbourne, Australia
}

Background: DriveSafe DriveAware (DSDA) is a cognitive screening iPad application designed to assess driving awareness and determine the need for more intensive on-road testing. Participants complete tests in DSDA and based on a scoring algorithm, DSDA categorizes participants into "likely to pass an on-road assessment," "likely to fail an on-road assessment," and "requires further testing." Participants who are categorized as "likely to pass" are not recommended to undertake expensive and time consuming on-road testing. Our research team previously studied DSDA in a Huntington's disease (HD) sample and found that DSDA miscategorized some participants as passing, despite having deficits in cognitive functioning that are expected to impair driving. We further examined whether variables within DSDA that are recorded, but not used, when determining categorizations could be utilized to improve its accuracy in triggering recommendations for on-road testing for people with HD. Specifically, we examined the time taken to complete the subtests and accuracy on the Intersection Rules subtest.

Methods: We used data previously collected by our research team from 26 participants with genetically confirmed HD CAG expansion and a clinical diagnosis of HD. We also recruited 26 healthy controls age and gender matched at a group level to the HD sample. All 52 participants completed DSDA and six neuropsychological assessments. The cognitive tests administered assess cognitive functions that have previously demonstrated their importance for safe driving.

Results: We found that the HD group completed DriveSafe and Intersection Rules subtests significantly slower than controls; however, no significant difference was found in accuracy on the Intersection Rules subtest. Time taken on subtests correlated with tests of processing speed, attention, planning, and visual perception, which are critical domains for safe driving. Accuracy on the Intersection Rules subtest correlated with a measure of visual perception.

Conclusions: The key findings that time taken in DSDA was associated with important skills for driving, and that the HD group was slower, suggest that time is important to consider when making appraisals of driving ability. It is recommended that clinicians consider the time taken in DSDA in conjunction with scores to make accurate recommendations about driving ability for people with HD that is reflective of their cognitive functioning.

\section{2}

Translatable Biomarkers in Gene Therapy for Huntington's Disease: Innovative Approaches and Learnings from Preclinic to the Clinic

Astrid Valles* ${ }^{1}$, Melvin M. Evers ${ }^{1}$, Anouk Stam ${ }^{1}$, Marina SogorbGonzalez ${ }^{1}$, Cynthia Brouwers ${ }^{1}$, Carlos Vendrell ${ }^{1}$, Seyda AcarBroekmans ${ }^{1}$, Valentina Fodale, Alberto Bresciani, Rienk Nieuwland ${ }^{2}$, Zdenka Ellederova ${ }^{3}$, Jan Motlik ${ }^{3}$, Martin de Haan ${ }^{1}$, Blair Leavitt ${ }^{4}$, Sander van Deventer ${ }^{5}$, Pavlina Konstantinova ${ }^{1}$

${ }^{1}$ uniQure, Institute of Animal Physiology and Genetics, Libechov, Czech Republic

${ }^{2}$ Amsterdam UMC, Amsterdam, Netherlands

${ }^{3}$ IAPG, Libechov, Czech Republic

${ }^{4}$ University of British Columbia, Vancouver, British Columbia, Canada

${ }^{5}$ Leiden University Medical Centre, Leiden, Netherlands

Background: Huntingtin (HTT)-lowering therapies hold great promise to slow down or halt neurodegeneration in Huntington's disease (HD). From the different approaches under development, gene therapies using adeno-associated viral vectors (AAVs) are typically administered locally into the brain region of interest, after which they expand to interconnected regions though different mechanisms. A single administration should be sufficient to ensure long-term persistence of the therapeutic transgene (i.e., lowering agent), especially in non-dividing cells such as neurons. We have developed AAV5-miHTT, a recombinant AAV-based gene therapy expressing an engineered microRNA that specifically binds to HTT exon1, resulting in lowering of both full-length and exon1 HTT 
mRNA expression. Now on its way to clinical development, AAV5miHTT has demonstrated safety and efficacy in small and large animal studies, with remarkable brain-wide spread and long-term persistence, through retrograde and anterograde transport of the AAV followed by extracellular spread of the therapeutic miRNA, and without off-target effects.

Methods: Adequate translational measures to evaluate the safety, efficacy, and durability of HTT lowering mediated by AAV5-miHTT in patients are much needed. To this end, we assessed the response of candidate biofluid and imaging biomarkers in preclinical models (from rodents to minipigs and non-human primates) to AAV5-miHTT administration. In cerebrospinal fluid (CSF), assessments included pharmacokinetic (miHTT expression) and pharmacodynamic (HTT protein, NFL) measures. Imaging biomarkers included volumetric magnetic resonance imaging (vMRI) and magnetic resonance spectroscopy (MRS). All experiments were performed in accordance with regulations from the Institute of Molecular Genetics.

Results: Data obtained from rodent and large animal models support the use of a biomarker panel that encompasses the combined use of biofluid and imaging measures. Promising measures to follow-up efficacy include expression of miHTT in CSF, and brain metabolites determined by MRS. Conclusions: Many of these measures have the potential to follow-up safety and efficacy of HTT-lowering therapies in general. However, because of the unique properties of gene therapy approaches in contrast to other HTT-lowering therapies, a tailored biomarker panel for HTTlowering gene therapies in HD patients may be needed.

\section{3}

Exploring the Effects of Intrastriatal AAV5-miHTT-Lowering Therapy on Transcriptional Dysregulation, MRS Signal, and Mutant Huntingtin Levels in the Q175FDN Mouse Model of Huntington's Disease

Blair Leavitt ${ }^{1}$, Anouk Stam ${ }^{2}$, Cynthia Brouwers ${ }^{2}$, Terri Petkau ${ }^{1}$, Michael Vermeulen ${ }^{1}$, Andrew Yung ${ }^{1}$, Bretta Russell-Schulz ${ }^{1}$, Piotr Kozlowski ${ }^{3}$, Alex Mackay ${ }^{4}$, Da Ma, Faisal Beg ${ }^{5}$, Sara Mostafavi ${ }^{6}$, Sander van Deventer $^{2}$, Melvin M. Evers ${ }^{2}$, Pavlina Konstantinova ${ }^{2}$, Astrid Valles*2

\footnotetext{
${ }^{1}$ University of British Columbia, Vancouver, British Columbia, Canada ${ }^{2}$ uniQure, Institute of Animal Physiology and Genetics, Libechov, Czech Republic

${ }^{3}$ Touro College of Osteopathic Medicine, New York, NY, USA

${ }^{4}$ Centre for Brain Health, Vancouver, British Columbia, Canada

${ }^{5}$ Simon Fraser University, Burnaby, British Columbia, Canada

${ }^{6}$ University of Washington, Seattle, WA, USA
}

Background: Huntingtin (HTT)-lowering therapies hold great promise to slow-down or halt neurodegeneration in Huntington's disease (HD). We have developed an engineered microRNA targeting human HTT, delivered via adeno-associated viral vector serotype 5 (AAV5-miHTT), leading to efficient HTT-lowering in vitro and in vivo in rodent models. Here, we explored the use of striatal RNA sequencing (RNAseq) and non-invasive magnetic resonance spectroscopy (MRS) as translational efficacy measures for the assessment of HTT-lowering therapies in HD.

Methods: Three-month-old homozygous Q175FDN HD mice were injected bilaterally into the striatum with formulation buffer (sham), low $(5.2 \times 109 \mathrm{gc} /$ mouse $)$ or high $(1.3 \times 1011 \mathrm{gc} /$ mouse $)$ doses of AAV5miHTT. Wild-type (WT) mice injected with formulation buffer served as controls. T1-weighted structural MR imaging (MRI) and striatal MRS were performed 3 months after injection, and shortly afterwards the animals were sacrificed to collect brain tissue for protein and RNA. Experiments were performed in accordance with regulations set by the Canadian Council on Animal Care.
Results: Decreased total N-acetylaspartate (tNAA, neuronal integrity marker) and increased myo-inositol (mI, gliosis marker) levels were found in Q175FDN sham-treated mice with respect to WT controls, similar to previous observations in the putamen of HD patients. These findings were reversed in the Q175FDN high-dose AAV5-miHTT-treated mice with higher levels of tNAA and reduced levels of mI compared to sham-treated Q175FDN mice. Dose-dependent changes in AAV5 vector DNA level, miHTT expression, and HTT protein were observed in the striatum and cortex. Correlations were shown between tNAA MRS levels and HTT protein levels in the striatum and cortex, suggesting a direct relationship between HTT lowering and the striatal MRS signal. Striatal transcriptional analysis using RNA seq revealed mHTT-induced changes that were partially reversed by HTT lowering.

Conclusions: Striatal MRS analysis suggests a restoration of neuronal function and partial reversal of gliosis, and RNAseq analysis shows a reversal of transcriptional dysregulation following AAV5-miHTT treatment. The results of this study support the use of MRS in HTT-lowering clinical trials and strengthen the therapeutic potential of AAV5-miHTT in reversing striatal dysfunction in HD.

\section{4}

Secreted Therapeutics: Monitoring Durability of microRNA-Based Gene Therapies in Huntington's Disease

Carlos Vendrell ${ }^{1}$, Jolanda Snapper ${ }^{1}$, Anouk Stam ${ }^{1}$, Jana Miniarikova ${ }^{2}$, Elisabeth A. Spronck ${ }^{1}$, Martin de Haan ${ }^{1}$, Rienk Nieuwland ${ }^{1}$, Pavlina Konstantinova $^{1}$, Sander van Deventer ${ }^{1}$, Melvin M. Evers ${ }^{1}$, Astrid Valles*1

${ }^{1}$ uniQure, Amsterdam, the Netherlands

${ }^{2}$ Leiden University, Leiden, the Netherlands

Background: MicroRNA-based gene therapies are emerging as a potential approach to lower huntingtin (HTT), the disease-causing protein of Huntington disease (HD). We have developed an engineered microRNA targeting human huntingtin (miHTT), delivered via adeno-associated serotype 5 virus (AAV5). AAV5-miHTT treatment has demonstrated an efficient lowering in vitro and in vivo in the brain in different HD animal models. One of the current translational challenges is the need for biomarkers that are indicative of the long-term expression and therapeutic efficacy of miHTT in the brain, where the therapy is directly infused. Recently, extracellular vesicles (EVs) have been identified as carriers of RNA species and proteins directly secreted from cells and present in all biofluids, becoming potential sources of biomarkers for diagnosis.

Methods: In this study, we investigated the detection of EV-associated miRNAs in biofluids as suitable measurements to monitor the expression and endurance of AAV-delivered miRNAs in the brain. Experiments were performed according to regulations set by the Netherlands Cancer Institute.

Results: iPS-derived neurons from HD patients confirmed the secretion of mature miHTT molecules within EVs. Moreover, EVenriched miHTT levels were strongly correlated with viral dose and transgene expression in neuronal cells. We further investigated the transability of extracellular miHTT to monitor the expression and durability of miHTT in vivo. Quantifiable miHTT levels enriched in EVs were detected in the cerebral spinal fluid in nonhuman primates up to 6 months and in HD transgenic minipigs up to 2 years after a single intrastriatal injection.

Conclusions: Altogether, these results confirm the long-term expression of AAV-delivered miRNAs and support the potential role of EV-associated miRNAs as a novel translational pharmacokinetic marker in gene therapies for central nervous system (CNS) disorders. 
55

\section{Lowering the Pathogenic Exon 1 HTT Fragment by AAV5-miRNA Gene Therapy}

Marina Sogorb-Gonzalez*, Fanny Mariet, Pavlina Konstantinova, Sander J. van Deventer, Melvin M. Evers, Astrid Valles

uniQure, Amsterdam, Netherlands (all authors)

Background: Huntington's disease (HD) is a genetic neurodegenerative disease caused by a trinucleotide repeat in the huntingtin gene (HTT). Despite its monogenic cause, HD pathology is highly complex and targeting the leading pathogenic mechanisms is relevant for developing disease modifying therapies. It has recently been demonstrated that apart from the mutant full-length HTT protein, exon 1 HTT fragments generated by aberrant splicing are very prone to aggregate and contribute to HD pathology. These findings suggest that approaches capable of reducing the expression of the highly pathogenic exon 1 HTT protein might achieve a greater therapeutic benefit than targeting only the full-length mutant HTT protein. We have developed an engineered microRNA targeting exon 1 HTT (miHTT), delivered via adeno-associated serotype 5 virus (AAV5). AAV5-miHTT treatment has been demonstrated to lower mHTT in several rodent and large animal models by reducing fulllength HTT and reversing HD phenotype.

Methods: In this study, we evaluated the ability of AAV5-miHTT to reduce the exon 1 HTT mRNA in knock-in mice. Experiments were performed in accordance with regulations set by the Netherlands Cancer Institute.

Results: Polyadenylated exon1 HTT mRNA was successfully detected in Q175FDN striatum and cortex by 3'RACE and qPCR. After intrastriatal administration of AAV-miHTT treatment, dose-dependent lowering of both full-length HTT and mis-spliced exon 1 HTT mRNA were detected in the striatum and cortex in Q175FDN mice.

Conclusions: These results suggest a new aspect to the potential therapeutic value of the first RNAi-based gene therapy for HD disease to enter clinical trials.

\section{6}

\section{Fully Automatic AI Segmentation of Subcortical Regions}

Jack Weatheritt* ${ }^{1}$, Richard Joules ${ }^{1}$, Robin Wolz ${ }^{1}$, Daniel Rueckert ${ }^{2}$

${ }^{1}$ IXICO, London, England, UK

${ }^{2}$ Imperial College London, London, England, UK

Background: Changes in volume of the putamen are used as biomarkers to track the development of Huntington's disease (HD) and monitor the potential effect of interventional treatments. Therefore, accurate volume calculations, obtained via segmentations, are of utmost clinical importance. These regions are particularly challenging for automated methodologies, due to high inter-subject variability and unclear region boundaries. We present a fully automated approach that does not require manual delineations of putamen for an HD population in order to be predictive. Methods: We present a deep-learning method, a 3D convolutional neural network $(\mathrm{CNN})$, which we tune for subcortical regions. The model is trained using data from an Alzheimer's disease (AD) cohort (ADNI), for whom variations in putamen are not considered pathological. Fully supervised training is performed by providing a neural-network-labeled ground truth data, from which to learn by example. The pipeline takes a T1 image as input, and the output is the putamen mask. All scans are registered to the MNI152 template and ground-truth labels are generated by correcting LEAP, an atlas-based approach, output. The AD cohort, consisting of 182 subjects, is split into a holdout set (20\%) and training data used for five-fold cross validation. The HD cohort, completely unseen during training, consists of 25 subjects, with a smaller mean combined putamen volume (5749.4 $\mathrm{mm} 3$ compared with $8848.1 \mathrm{~mm} 3$ for the $\mathrm{AD}$ cohort, $p$ value $<0.05$ ) used to test the pipeline. Our method is compared against an established atlas-based approach (MALPEM) on the HD cohort.

Results: After the initial learning phase, the model is shown to generalize to the new HD cohort, without specific adjustment to account for the reduced anatomical volume or scanner protocol. Error in volume calculations are homoscedastic. When comparing to MALPEM, the CNN performs very favorably. The standard deviation of volume error (considering both structures together) for both methodologies is comparable: $719.3 \mathrm{~mm} 3$ for the CNN, against $742.9 \mathrm{~mm}^{3}$ for MALPEM. However, MALPEM has a systematic tendency to under segment the HD putamen. The mean volume error for MALPEM is $1983.3 \mathrm{~mm}^{3}$; this is contrasted with a mean error of $223.4 \mathrm{~mm}^{3}$ for the $\mathrm{CNN}$.

Conclusions: Our deep-learning pipeline can be trained on non-HD subjects and then used on an HD population. This is promising because the difference in putamen volume is high, and representative databases for algorithm development may not exist. This goes against common wisdom that neural networks do not generalize well for differing data distributions. Further, the comparison with the atlas-based approach is highly favorable, indicating that high performance on this data set is not trivial.

\section{7}

Characterizing Brain Structure in HD Gene Carriers 24 Years from Clinical Onset: the Young Adult Study

Rachael Scahill*1, Paul Zeun ${ }^{1}$, Eileanoir B. Johnson ${ }^{1}$, Sarah Gregory ${ }^{1}$, Christopher Parker ${ }^{1}$, Katherine Osborne-Crowley ${ }^{1}$, Jessica Lowe ${ }^{1}$, Peter McColgan $^{1}$, Akshay Nair ${ }^{1}$, Marina Papoutsi ${ }^{1}$, Carlos Estevez-Fraga ${ }^{1}$, Kate Fayer ${ }^{1}$, Harpreet Hyare ${ }^{1}$, Cristina Sampaio ${ }^{2}$, Edward J. Wild, Trevor W. Robbins, Barbara J. Sahakian, Hui Zhang ${ }^{1}$, Geraint Rees ${ }^{1}$, Douglas Langbehn ${ }^{4}$, Sarah J. Tabrizi ${ }^{1}$

${ }^{1}$ University College London, London, UK

${ }^{2}$ CHDI Foundation, Los Angeles, CA, USA

${ }^{3}$ University of Cambridge, Cambridge, UK

${ }^{4}$ University of Iowa, Iowa City, IA, USA

Background: Differences in brain structure are present in HD gene carriers up to 15 years before the onset of clinical symptoms, and recent work suggests that the HD gene may even exert an effect during development. We aimed to establish whether there were detectable structural brain differences in our Young Adult Study participants (HD-YAS), the earliest adult HD cohort studied to date.

Methods: Sixty-two premanifest (PreHD) participants approximately 24 years from expected disease onset and 61 controls underwent volumetric T1, diffusion imaging using a neurite orientation and dispersion density imaging (NODDI) sequence and multiparametric mapping (MPM). We derived measures of GM volume, myelin, iron, white matter microstructure, and structural connectivity and used general least-squares linear models to assess group differences and association with age and CAG. Results were corrected for multiple comparisons using the false discovery rate (FDR).

Results: The PreHD cohort had slightly smaller putamen volumes ( $q=$ $0.03)$ and caudate volume was reduced $(p=0.05)$, although this did not survive FDR correction $(q=0.20)$. No other brain metrics survived FDR correction $(q>0.16)$, and there was no association with disease burden $(q>0.48)$. There were subthreshold disease-related differences in white matter microstructural measures in the corpus callosum $(p=0.007)$ and internal capsule $(p=0.04)$. MPMs demonstrated altered R1 signal in the putamen $(p=0.005)$ and external capsule $(p=0.05)$ and R2 signal in the 
posterior internal capsule $(p=0.02)$, external capsule $(p=0.02)$, and the putamen $(p=0.03)$.

Conclusions: With the exception of small volume reductions in the striatum, there was little evidence that carrying the HD gene has a substantial impact on brain volume, microstructure, or structural connectivity at this stage of the disease; this is supported by the lack of an association between these measures and age and CAG. Nevertheless, it is possible that there are some subtle changes occurring that are suggestive of the start of the neurodegenerative process, with subthreshold changes in white matter microstructure and alterations in R1 and R2 signal that may be indicative of iron accumulation.

58

Validity, Reliability, and Ability to Detect Change of the Total Functional Capacity Score in a GENERATION HD1-like Huntington's Disease (HD) Population

Dylan Trundell*1, Jestinah Chevure, Susanne Clinch $^{1}$, Simona Rossomanno $^{1}$, Scott A. Schobel ${ }^{1}$, G. Bernhard Landwehrmeyer ${ }^{2}$, Blair R. Leavitt ${ }^{3}$, Karen Anderson ${ }^{4}$

${ }^{1}$ F. Hoffmann-La Roche Ltd., Basel, Switzerland

${ }^{2}$ University of Ulm, Ulm, Germany

${ }^{3}$ University of British Columbia, Vancouver, British Columbia, Canada

${ }^{4}$ Georgetown University, Washington, D.C., USA

Background: Evidence of favorable psychometric properties is important for supporting the use of outcome assessments in longitudinal research, such as interventional clinical trials. The unified HD rating scale-total functional capacity (TFC), a clinician-reported assessment, is the US primary endpoint in GENERATION HD1 (NCT03761849), an ongoing, randomized, placebo-controlled phase III study assessing the efficacy and safety of tominersen in individuals with manifest HD. Although the psychometric properties have been assessed in a broader manifest population, they have not previously been assessed in a GENERATION HD1-like population. The aim of this retrospective analysis was to identify the validity, reliability, and ability to detect change of the TFC in a GENERATION HD1-like population, using data from a multinational registry.

Methods: The analysis sample from Enroll-HD, a multinational, longitudinal, observational registry study included individuals who met the following criteria (consistent with GENERATION HD1): 25-65 years, CAG-age product $>400$, independence scale (IS) $\geq 70$, and diagnostic confidence level $=4$. Sociodemographic descriptive statistics were calculated to characterize the sample. Test-retest reliability was assessed by intraclass correlation coefficient (ICC) in a subset of patients with no change in IS score. Internal consistency was assessed using Cronbach's alpha $(\alpha)$. Convergent validity was assessed by Spearman's rank-order correlation coefficient $(\rho)$ with other measures. Known-groups validity and ability to detect change was assessed by analysis of covariance, comparing TFC scores/change scores between groups defined by IS and functional assessment (FA) score/change score.

Results: Good evidence of reliability was demonstrated (ICC $=0.89 ; \alpha=$ $0.74)$. Convergent validity was supported by a magnitude and pattern of correlations in line with a priori expectations (e.g., FA: $\rho=0.9$; IS: $\rho=$ 0.8 ; Total Motor Score: $\rho=-0.5)$. Significant differences $(p<0.001)$ were identified for all known groups and ability to detect change analyses, demonstrating that the TFC was able to distinguish between groups known to differ in both cross-sectional functioning/independence status and longitudinal functioning/independence outcomes.

Conclusions: Evidence supporting the validity, reliability, and ability to detect change of the TFC was identified in a GENERATION HD1-like population, supporting the use of this outcome assessment in the ongoing clinical trial.
59

Exposure-Response Analysis Demonstrates Predicted Exposure for Clinical Efficacy with Pridopidine 45-mg Bid Dose

Michal Geva*1, Andrew McGarry ${ }^{2}$, Noga Gershoni-Emek ${ }^{3}$, C. Warren Olanow $^{4}$, Karl Kieburtz ${ }^{5}$, Michael R. Hayden ${ }^{6}$

${ }^{1}$ Prilenia Neurotherapeutics, Herzliya, Israel

${ }^{2}$ Cooper University Hospital, Cherry Hill, NJ, USA

${ }^{3}$ Tel Aviv University, Tel Aviv, Israel

${ }^{4}$ Mount Sinai School of Medicine, New York, NY, USA

${ }^{5}$ University of Rochester, Rochester, NY, USA

${ }^{6}$ University of British Columbia, Vancouver, British Columbia, Canada Background: Pridopidine is a novel small molecule in clinical development for HD and ALS with high affinity and selective agonistic activity at the Sigma-1 Receptor (S1R). The S1R is an ER protein located at the mitochondrial interface, modulating diverse cellular processes. S1R activation by pridopidine induces beneficial effects in multiple neurodegenerative disease models. S1R agonist activity is characterized by a bellshaped dose response curve in both pre- and clinical studies. Bell-shaped dose response is defined as a beneficial effect seen at low doses, which is reduced at higher doses. Pridopidine demonstrates bell-shaped effects in several preclinical models. A bell-shaped response curve with an optimal dose of $1 \mu \mathrm{M}$ is observed for BDNF secretion in B104 neuroblastoma cells, as well as for restoration of homeostatic synaptic plasticity in YAC128 HD cortical neurons. Similarly, in the 6-OHDA PD mouse model, pridopidine low dose was more efficacious than high dose $(0.3$ vs. $1 \mathrm{mg} / \mathrm{kg}$ ), demonstrating survival of dopaminergic neurons and behavioral rescue. Pridopidine $45 \mathrm{mg}$ bid is the most efficacious dose in clinical trials. In HART and MermaiHD, $45 \mathrm{mg}$ bid, the highest dose tested, was most efficacious for improving Total Motor Score (TMS). In the PRIDE-HD trial, the lowest dose tested, $45 \mathrm{mg}$ bid was the most efficacious for maintaining Total Functional Capacity (TFC). These data adhere to the expected S1R agonist bell-shaped response.

Methods: To evaluate pridopidine exposure-response relationship for TFC, exposure (AUC) was plotted against TFC score at 52 weeks per individual in PRIDE-HD.

Results: We show a correlation between plasma exposure levels and TFC change from baseline. Plasma concentrations of AUC 7-19 mg*h/ml were associated with no deterioration or improvement of TFC $(\triangle \mathrm{TFC} \geq 0)$. Within the $45 \mathrm{mg}$ bid treatment group, $94 \%$ of patients had AUC $7-19 \mathrm{mg} * \mathrm{~h} / \mathrm{ml}$, and of these, $82 \%$ show no deterioration or improvement in TFC. AUC 7-19 $\mathrm{mg} * \mathrm{~h} / \mathrm{ml}$ was measured in small numbers at doses higher than $45 \mathrm{mg}$ bid $(30 \%, 12 \%$, and $4 \%$ of the $67.5,90$, and $112.5 \mathrm{mg}$ bid groups, respectively). At higher doses combined, the majority of trial participants (61.5\%) with AUC 7-19 mg*h/ml also demonstrate no deterioration or improvement in TFC. However, at higher doses, where most patients had AUC $\geq 20 \mathrm{mg} * \mathrm{~h} / \mathrm{ml}$, most patients show deterioration in TFC.

Conclusions: A 45-mg bid dose of pridopidine yields the predicted exposure for clinical efficacy, measured by TFC.

\section{0}

\section{Enroll-HD Platform Data Resources}

Mette Gilling*, Enroll-HD platform

CHDI Foundation, Inc. New York, USA (all authors)

Enroll-HD is a clinical research platform that includes at its core a global observational study of Huntington's disease (HD) families who are followed annually. Currently, the Enroll-HD study includes over 23,800 
participants (more than 19,750 active participants) from 20 countries in Europe, North America, Latin America, and Australasia. Enroll-HD provides high-quality coded clinical data and biosamples to qualified researchers in the HD research community via a straightforward request process (https://enroll-hd.org/for-researchers/). Every 1-2 years, an easy access Enroll-HD dataset (periodic dataset, PDS) that includes approximately $80 \%$ of the variables collected in the study is prepared and made available to qualified HD researchers within 3 days of request. The next Enroll-HD PDS release is planned for December 2020; to date, the various Enroll-HD PDS datasets have been downloaded for 228 different projects. The risk for participant identification from the PDS is low, but if researchers request any of the remaining $20 \%$ of the collected variables, the risk for participant identification may be increased, and, therefore, a specified dataset request must be reviewed and approved by the EnrollHD Scientific Review Committee (SRC). When the SRC reviews SPS requests they consider whether the potential scientific outcome of the project outweighs the increased risk for participant identification. When approved by the SRC, an SPS dataset can be prepared and released. To date, more than 80 specified dataset requests for clinical data have been delivered. In addition to Enroll-HD, clinical data can be requested from the studies' Registry (14,522 participants), HDClarity (currently approximately 500 participants) and TRACK-HD/ON (approximately 400 participants). A large, easy-access registry dataset (RDS) prepared in a format similar to the Enroll-HD PDS can augment the Enroll-HD PDS and thereby increase the total number of participants for modeling purposes. The RDS can be requested by contacting the EHDN Scientific Bioethics Advisory Board (SBAC). Datasets are prepared free of charge. In addition to clinical data, the EnrollHD platform distributes smaller imaging, brain morphometric/volumetric, GWAS, RNAseq, MiSeq, methylation, and proteomics datasets collected across 10 studies. New datasets are constantly added from new clinical studies or as outputs from the available biosample resources.

\section{1}

\section{Enroll-HD Platform Biosample Resources}

Mette Gilling*, Enroll-HD platform

CHDI Foundation, Inc., New York, USA

Enroll-HD is a clinical research platform that includes at its core a global observational study of Huntington's disease (HD) families who are followed annually. Currently, the Enroll-HD study includes over 23,800 participants (more than 19,750 active participants) from 20 countries in Europe, North America, Latin America, and Australasia. In its capacity of clinical research platform, Enroll-HD provides high-quality coded clinical data and biosamples to qualified researchers in the HD research community via a straightforward request process (https://enroll-hd.org/forresearchers/). Due to its longitudinal nature, more than 58,000 blood samples have been collected in Enroll-HD to date. Currently, 16 different types of biosamples collected in five different studies (Enroll-HD, HDCSF/ HDClarity, HDYAS, TRACK-HD/TRACK-ON, MTM-HD) are available via the Enroll-HD platform and additional biosample collections are in the planning stage. To date, more than $70 \mathrm{HD}$ research projects have used Enroll-HD platform biosample resources to support their research. Non-renewable biosample resources require review and approval by the Enroll-HD Scientific Review Committee (SRC) before release, whereas renewable resources can be released without SRC review. All biosample distributions come with a material, shipping, and handling fee, and a material transfer agreement must be signed before biosamples can be shipped. Currently available biosample resources: Lymphoblastoid cell lines (LCLs), DNA from LCLs, DNA from whole blood, PBMCs, buffy coat, EDTA plasma, LiHep plasma, CSF, cells from CSF, Serum, PAX gene RNA, buccal swabs, fibroblasts, myoblasts, primary fat, and iPS cells.

\section{2}

Clustering and Prediction of Disease Progression Trajectories in Huntington's Disease: an Analysis of Enroll-HD and REGISTRY Data Using a Machine-Learning Approach

Jinnie Ko*1 ${ }^{1}$, Hannah Furby ${ }^{2}$, Xiaoye Ma ${ }^{1}$, Jeffrey D. Long ${ }^{3}$, Xiao-Yu Lu, Diana Slowiejko ${ }^{1}$, Rita Gandhy ${ }^{1}$

${ }^{1}$ Genentech, Inc., South San Francisco, CA, USA

${ }^{2}$ Roche Products Ltd., Welwyn Garden City, UK

${ }^{3}$ University of Iowa, Iowa City, IA, USA

Introduction: Huntington's disease (HD) is a rare, genetic, neurodegenerative disease characterized by cognitive and motor decline, and by behavioral symptoms. Cytosine adenine guanine (CAG) repeat length and the age of symptom onset are strong predicting factors of disease progression; however, the holistic impact of factors, including clinical presentation, family history, and social factors, are not well understood. Our aim is to quantitatively describe HD progression trajectories jointly for symptoms associated with multiple clinical domains, and to identify features predictive of progression trajectory using data from individuals with manifest HD.

Methods: Combined data from the European HD Network prospective study (REGISTRY) and Enroll-HD, a longitudinal, observational registry study, were used. Participants with manifest HD and $\geq 2$ annual visits were included $(N=4561)$. A $K$-means method for joint longitudinal trajectories was used to cluster participants based on similarities in progression trajectories to capture multiple clinical domains (including motor, cognitive, and behavioral domains). Impactful features that predicted trajectory membership were identified by the gradient-boosting machines algorithm out of a pool of demographic, genetic/family history, social, symptom, medication, and clinical factors.

Results: Participants with manifest HD were grouped into three clusters (rapid, moderate, or slow progression) based on joint longitudinal trajectories of Total Motor Score, Symbol Digit Modalities Test, and Apathy scores. The top 15 predicting features were measures of age at rater's diagnosis; CAP (CAG-age product); body mass index; care partner status; education level; CAG repeat length; family history; medical histories of apathy; cognitive impairment; perseverative, obsessive behavior; psychosis; use of tetrabenazine, antipsychotics-benzamides, antipsychotics; and region (North America vs. others).

Conclusions: By assessing progression jointly across clinical outcomes, we demonstrate that individuals with manifest HD can be clustered by age, social, clinical, and genetic variables. The clustering provides a profile for different rates of progression, which may be useful to guide personalized patient care and management plans.

\section{3}

\section{Quantification of Mutant Huntingtin Protein (mHTT) in Human Cerebrospinal Fluid to Support Clinical Development of mHTT- Targeting Therapies in Huntington's Disease}

Katharina Schutz, Alessandra Buehler, Eginhard Schick, Marianne Manchester Young, Lauren Boak, Scott A. Schobel, David J. Hawellek*

F. Hoffmann-La Roche, Ltd., Basel, Switzerland (all authors)

Introduction: Huntington's disease (HD) is a rare, genetic neurodegenerative disease caused by a CAG repeat expansion in the huntingtin gene 
(HTT), resulting in the production of toxic mutant huntingtin protein (mHTT). Current therapeutic approaches include lowering mHTT; therefore, quantification of mHTT in the cerebrospinal fluid (CSF), using a method that is compliant with Good Clinical Practice (GCP) guidelines, is critical for producing robust mHTT clinical trial data to support the clinical development of mHTT-targeting therapies. Here, we report the results of the optimization and validation of a bioanalytical method for the reliable quantification of mHTT protein levels in human CSF.

Methods: All results were generated in a regulated bioanalytical environment (i.e., by GCP-trained personnel in a Good Laboratory Practicecertified laboratory) using a bead-based sandwich ligand-binding assay with Single Molecule Counting detection on the SMCxPROTM (Merck). The ultra-sensitive assay employs the antibody pair 2B7/MW1 for capture and detection and artificial CSF as a surrogate matrix. Assay validation followed international guidelines adapted in specific aspects based on prevalidation experiments.

Results: A comparison of nine different recombinant HTT proteins (varying in fragment length and polyglutamine length) during prevalidation revealed excellent discriminability between wild-type HTT (wtHTT) and mHTT. The calibration curves for all mutant proteins were parallel, suggesting an equivalent performance as standard for relative quantitation of mHTT levels. The assay validation confirmed robust inter- and intraassay accuracy and precision, as well as parallelism and a high sensitivity (lower limit of quantification $=1.57 \mathrm{pg}$-eq $/ \mathrm{mL}$ ). We observed an absence of a prozone effect and no interference through factors potentially related to the conduct of clinical trials (blood, wtHTT, study drug [tominersen]). Our experiments also helped in mapping the limitations of the assay. Conclusions: This assay is a reliable, GCP-compliant bioanalytical method for measuring changes in mHTT levels in human CSF and is deemed suitable for use in registrational clinical trials investigating mHTTlowering therapies.

\section{4}

\section{Neurofilament Light Protein as a Blood-Based Biomarker for Juvenile-Onset Huntington's Disease}

Lauren Byrne $*^{1}$, Jordan L. Schultz ${ }^{2}$, Filipe B. Rodrigues ${ }^{1}$, Ellen van der Plas $^{1}$, Douglas Langbehn ${ }^{1}$, Peggy C. Nopoulous ${ }^{2}$, Edward J. Wild ${ }^{1}$

${ }^{1}$ University College London, London, UK

${ }^{2}$ The University of Iowa, Iowa City, IA, USA

Background: There is an acute need for disease-modifying therapies for juvenile-onset Huntington's disease (JoHD), a rare and devastating subtype of Huntington's disease (HD) where the onset of symptoms occurs before the age of 21. However, there is a lack of established biomarkers or clinical outcome measures for JoHD to implement clinical trials in this complex disease. Neurofilament light protein (NfL) in blood has emerged as a prognostic biomarker for HD but has not been quantified in JoHD or childhood premanifest HD mutation carriers.

Methods: We quantified NfL using ultrasensitive Simoa technology in blood plasma samples from children without the HD mutation (Controls, $n=60$ ); children and young adults with the HD mutation with HD symptoms (JoHD, $n=9$ ); and children without symptoms (preHD, $n=30$ ) from the KidsHD and KidsJHD cohorts. Linear mixed-effects regression analyses were used to assess group differences in plasma NfL and its associations with continuous measures. Years to predicted motor onset (YTO) were used to investigate $\mathrm{NfL}$ in preHD.

Results: JoHD patients had significantly higher plasma NfL than controls. NfL had a positive association with disease burden score in JoHD. PreHD children within 15 YTO had significantly higher NfL than controls. NfL was also elevated compared to controls in the 15-20 YTO group.
Conclusions: We report the first blood-based biomarker for JoHD that could eventually be used to facilitate clinical trials for this disease group. Elevated NfL in preHD children within 20 YTO is consistent with reports that NfL is among the earliest detectable changes in HD.

65

Geographic Clusters of Huntington's Disease in Northwestern Ceará: a Preliminary Analysis

Luís Edmundo Teixeira de Arruda Furtado*1, Sarah Arruda Furtado Carneiro ${ }^{2}$, Augusto César Cardoso dos Santos ${ }^{3}$, Samir Câmara Magalhães ${ }^{4}$, José Renan Miranda Cavalcante Filho ${ }^{4}$

${ }^{1}$ UNINTA, Sobral, Ceará, Brazil

${ }^{2}$ Faculdade de Medicina de Jundiaí, Jundiaí, SP, Brazil

${ }^{3}$ Brazilian Ministry of Health, Brasília, DF, Brazil

${ }^{4}$ Universidade Federal do Ceará, Fortaleza, Brazil

Background: The prevalence of Huntington's disease (HD) in Brazil is unknown. There are a few hospital-based studies, which were mostly designed to analyze clinical and molecular aspects of patients or some geographically delimited populations. Epidemiological studies in the Northeast region of Brazil are even more scarce, although this region has a high concentration of clusters of genetic diseases and congenital anomalies. Our study aimed to explore the geographic distribution of families with HD in the Northwest region of the state of Ceará. In particular, the northwestern region of Ceará is formed by 47 municipalities.

Methods: We searched for the terms Huntington, chorea, and ICD 10 G10 in medical records at the regional Neurology Centers. The activities carried out during an active search were: preparation of heredograms, genetic counseling, and neurological and dental consultations, in addition to audiovisual recording. In field research, the Senador Sá, Cruz, and Ibiapaba agglomerations were visited. The Pires Ferreira cluster has not yet been visited. In Senador Sá, the study was fully completed.

Results: Four possible agglomerations of HD were identified in the municipalities of Pires Ferreira, Cruz, Senador Sá, and Ibiapaba. Senador Sá (human development index [HDI]: 0.603) has 6.852 inhabitants distributed in a central region and two districts: Serrota and Salão. The latter was formed by 454 inhabitants, of which 16 were clinically identified as carriers of HD (23.3/10.000 inhabitants). The district is isolated and only received a paved road in the mid-1980s and is known locally as a "City of Cousins" due to the high number of consanguineous unions. Interestingly, Salão revealed another particularity: nowadays, DH is known as "bole," a word related to the verb "bulir," which means to stir. In Cruz (HDI: 0.632; 22.479 inhabitants), five carriers in the districts of Aroeira and Preá (2.23/10.000) were detected. Ibiapaba is the largest area studied (320.838 inhabitants), covering eight municipalities, where 13 inhabitants were identified in six different municipalities (0.4/10.000). The Pires Ferreira agglomeration (HDI: 0.606; 10.216 inhabitants) has not yet been visited, but in search of medical records, three people from the area known as Tabuleiro (2.9/10.000) were found.

Conclusions: Four possible geographic clusters of HD were found in the northwestern region of $\mathrm{CE}$, which prompted further investigations to verify such occurrences.

\section{6}

\section{Enroll-HD Platform Support for Industry and Academic Sponsors}

Jenny Townhill*, Enroll-HD Platform Team

CHDI Foundation, New York, United States (all authors)

Enroll-HD is a global research platform with the infrastructure to support clinical trials and studies in Huntington's disease (HD). At the core of the 
platform is the Enroll-HD study, a prospective, observational, longitudinal study of HD (see separate poster). Over 23,800 participants (more than 19,750 currently active) have been recruited into the Enroll-HD study, and have completed standardized clinical assessments and biosample collections at annual visits at 173 study sites (158 currently active) in 20 countries. Resources available to the HD research community include easily accessible periodic clinical datasets and biosamples (see separate posters); assistance with study and site feasibility and identification; protocol development and protocol advisory review, via expert panels; participant identification and recruitment; and site staff training and certification via the Enroll-HD training portal. Long-term close working relationships with the clinical sites, facilitated by operational management of the Enroll-HD study, allows for well-informed site identification and efficient feasibility based on extensive knowledge of site capabilities, historic performance of sites, and information about access to potential participants. The Enroll-HD participant database allows for powerful insilico screening, using study-specific inclusion and exclusion criteria to identify potentially eligible participants at clinical trial sites that can guide and support recruitment. This site intelligence is supported by the EnrollHD HD Clinical Trial Site Certification scheme, which encompasses Enroll-HD and non-Enroll-HD countries and sites, where sites are assessed against a set of standard minimum criteria for clinical trial participation. These relationships with sites also enable ongoing support with issue resolution and trial facilitation, where required.

67

Presence of Pre-existing Antiadeno-Associated Virus (AAV) Rerotype 5 Neutralizing Antibodies (NABs) in Serum of Huntington's Disease (HD) Patients Was Not Associated with Detectable AntiAAV5 NABs in Cerebrospinal Fluid (CSF)

Anna Majowicz* ${ }^{1}$, Floris van Waes ${ }^{1}$, Astrid Valles ${ }^{1}$, Sander J.H. van Deventer ${ }^{2}$, Mette Gilling ${ }^{3}$, Anka G. Ehrhardt ${ }^{4}$, Pavlina Konstantinova ${ }^{1}$, Valerie Ferreira ${ }^{1}$

${ }^{1}$ uniQure, Amsterdam, the Netherlands

${ }^{2}$ Leiden University Medical Centre, Leiden, Netherlands

${ }^{3}$ European Huntington's Disease Network, Copenhagen, Denmark

${ }^{4}$ CHDI Foundation, New York City, NY, USA

Background: AAV-based therapies are under investigation in early clinical trials for several neurodegenerative diseases. We have previously reported that serum antiAAV5 NABs titers up to 340 in humans and as high as 1030 in primates did not interfere with the therapeutic efficacy of intravenously administered AAV5 vector. However, it remains unclear whether naturally acquired pre-existing systemic immunity to AAV would impact the therapeutic efficacy of AAV vectors delivery to the central nervous system (CNS). This knowledge is of importance for the interpretation of AAV-based gene therapies for Huntington's disease (HD), since the blood-brain barrier in HD patients might be compromised. To address such concern, the prevalence of NABs against AAV serotype 5 was correlated between matched serum and CSF samples obtained from the same individual, either HD patients at different stages of the disease, or healthy controls participating in HDClarity.

Methods: Serum and plasma samples from HD patients and healthy donors were analyzed for the presence of antiAAV5 NABs with the use of a luciferase-based assay. The sensitivity (lowest sample dilution) of the assay was set at 50. Consequently, CSF samples were obtained from all HD patients and healthy donors that tested positive for serum/plasma antiAAV5 NABs and three that tested negative (controls) to analyze for presence of CSF antiAAV5 NABs.

Results: Overall, serum antiAAV5 NABs were not associated with detectable NABs in CSF; remarkably, this was true even in the presence of the highest serum titers (5364 in HD patient, 5129 in healthy donor). In
HD patients, 37 (21.5\%) of 172 serum samples had detectable antiAAV5 NAB titers; none $(0 \%)$ of the $37 \mathrm{HD}$ patients had detectable antiAAV5 NAB titers in CSF above threshold. Similarly, in healthy controls, 12 (18.2\%) of 66 serum samples had a detectable antiAAV5 NABs titer; none $(0 \%)$ of those 12 donors had detectable antiAAV5 NABs titers in CSF.

Conclusions: Pre-existing serum antiAAV5 antibodies have not impacted clinical efficacy of intravenous AAV5 therapies. The current analysis of HDClarity samples suggest patients with detectable serum NABs do not have CSF NABs. Therefore, we conclude that there is minimal risk for reduced therapeutic efficacy of intrathecal or intraparenchymal administration of therapeutic AAV5 vectors by pre-existing serum or CSF AAV5 NABs in HD patients.

\section{8}

Behavioral Benefit in YAC128 and BACHD Mouse Models of Huntington's Disease with Reduced Human HTT Protein Following Intrastriatal Administration of VY-HTT01 Gene Therapy

Pengcheng Zhou* ${ }^{1}$, Bin Liu ${ }^{2}$, Fen Chen ${ }^{1}$, Jianyu Shang ${ }^{1}$, Nilesh Pande ${ }^{1}$, Katherine Tyson ${ }^{1}$, Kelsey Bitner ${ }^{1}$, Todd Carter $^{1}$, Dinah Sah ${ }^{1}$, Kelly Bales $^{1}$, Omar Khwaja ${ }^{1}$

${ }^{1}$ Voyager Therapeutics, Inc., Cambridge, MA, USA

${ }^{2}$ Biogen, Chestnut Hill, MA, USA

Background: Lowering mutant HTT protein has the potential to slow or stop clinical progression of Huntington's disease (HD). VY-HTT01 is an adeno-associated virus, serotype-1 (AAV1), nonallele-specific gene therapy encoding a primary miRNA targeting human HTT (HsHTT) mRNA in development as an HTT-lowering therapy for HD.

Methods: YAC128 and BACHD mice received bilateral intrastriatal injections of vehicle or a low, medium, or high dose of VYHTT01 at $2.5 \mathrm{~m}$ of age. In-life and behavioral observations occurred over 24 (YAC128; Rotarod) or 16 weeks (BACHD; Rotarod, light/dark box, balance beam, open field), with pharmacology for HsHTT mRNA and protein at 16 weeks in both models and 24 weeks in YAC128, and histology for neurotoxicity at 24 (YAC128) and 16 (BACHD) weeks.

Results: VY-HTT01 significantly lowered human HsHTT mRNA relative to vehicle control in both models at all dose levels. Analysis of HsHTT protein showed a dose-dependent lowering at $16 \mathrm{w}$ in both models (YAC128: $-38 \%,-52 \%$, and $-65 \%$ for low, medium, and high doses; BACHD $-47 \%,-66 \%,-78 \%$, respectively) which maintained through $24 \mathrm{w}$ in the YAC128 model $(-47 \%$, $-50 \%,-71 \%$, respectively). There was a statistically significant increase in latency to fall in the YAC128 mice at $16 \mathrm{w}$; there was no improvement in the BACHD mice in latency to fall. In BACHD mice at $16 \mathrm{w}$, there were numerical decreases in balance beam time to cross that was significant in the medium-dose group but did not reach statistical significance for the low- or high-dose groups relative to vehicle control; there were no changes in light/ dark box or open-field behavior. Assessment of neurotoxicity in the striatum showed no changes in NeuN-positive neuron density or Iba1I-positive microglia density, no treatment-associated GFAP-positive astrocyte proliferation, and no neuronal degeneration (Fluoro-Jade C) in both mouse models of HD.

Conclusions: In two mouse models of HD, there were significant decreases in HsHTT mRNA and protein at 16 weeks after administration of VY-HTT01 that were maintained through 24 weeks in the YAC128 model (not tested in BACHD); significant rescue of motor deficits was also observed in these animals. VY-HTT01 was well tolerated with no evidence of neurotoxicity in the assessed striatal tissues. 
69

\section{Stereopure Oligonucleotides for the Selective Silencing of Mutant Huntingtin}

Elena Dale ${ }^{* 1}$, Maria Frank-Kamenetsky ${ }^{1}$, Kristin Taborn ${ }^{1}$, Kidist Aklilu $^{2}$, Yuanjing Liu ${ }^{1}$, Shaunna Berkovitch ${ }^{1}$, Jeffrey Brown ${ }^{1}$, Pachamuthu Kandasamy ${ }^{1}$, Jayakanthan Kumarasamy ${ }^{1}$, Naoki Iwamoto $^{1}$, Yuan Yin, Hailin Yang ${ }^{1}$, Stephany Standley ${ }^{1}$, Frank Favaloro $^{1}$, Kenneth Longo ${ }^{1}$, Michael Byrne ${ }^{1}$, Chandra Vargeese ${ }^{1}$

${ }^{1}$ Wave Life Sciences, Cambridge, MA, USA

${ }^{2}$ Lucy Therapeutics, Boston, MA, USA

Background: Huntington's disease (HD) is caused by CAG repeat expansion ( $\geq 36$ repeats) in the HTT gene that produces mHTT protein. The wild-type HTT protein is important for normal neuronal homeostasis and survival, so its suppression may have detrimental long-term consequences. Oligonucleotides that target the mutant variant of a heterozygous SNP in HTT can elicit selective activity by promoting RNase Hmediated degradation of mHTT transcripts. Wave Life Sciences' oligonucleotides are designed using PRISMTM, our proprietary discovery and drug development platform, which combines our growing knowledge of how the interplay among oligonucleotide sequence, chemistry, and backbone stereochemistry impacts key pharmacologic properties.

Methods: Biochemical RNase $\mathrm{H}$ experiments were performed with synthetic mHTT and wtHTT RNA substrates, stereopure oligonucleotides, and purified catalytic domain from RNase H1. Oligonucleotides were delivered to cultured, patient-derived iCell neurons (heterozygous and homozygous) under free-uptake conditions. In vivo efficacy and distribution studies were performed in BACHD transgenic mice carrying the human HTT gene with a repeat expansion and SNP of interest.

Results: In biochemical experiments, Wave's stereopure compounds led to degradation of mHTT while largely sparing wtHTT RNA. Wave's stereopure oligonucleotides showed potent knockdown of mHTT RNA in iCell neurons homozygous for the SNP and promoted mHTT RNA degradation in heterozygous iCell neurons. In BACHD mice, stereopure oligonucleotides engaged mHTT RNA, which led to a durable knockdown of mHTT in the cortex and striatum.

Conclusions: In preclinical studies, Wave's stereopure oligonucleotides targeting a heterozygous SNP in HTT led to selective knockdown of mHTT in vitro, while leaving wtHTT largely spared. These stereopure oligonucleotides also led to durable knockdown of mHTT in vivo.

70

HDBOI: Quantifying the Socio-Economic Burden of Huntington's Disease in the USA and EU5-Study Design and Expert Inputs

Nanxin (Nick) Li ${ }^{1 *}$, Leonardo Ruiz

HCD Economics, Warrington, UK (all authors)

Background: The burden associated with Huntington's disease (HD) is substantial. However, limited evidence is available on comprehensive assessment of clinical, economic, and humanistic burden of HD from a multinational perspective. There is also a need to analyze burden across all stages of HD to bring important insight on possible savings associated with the introduction of disease-modifying therapies. This HD burden of illness (HDBOI) study will assess the overall clinical, economic, and humanistic burden of HD experienced by patients, caregivers, health systems, and society. This analysis will be performed across all stages of HD and for the different countries in scope (US \& EU-5).
Methods: The study will collect retrospective and cross-sectional data at the patient, caregiver, and physician-level in multiple centers across the USA and EU-5. Physicians will provide sociodemographic, clinical, and medical resource utilization using a web-based electronic case record form (eCRF). Patients and caregivers will additionally provide further information on economic and quality of life (QoL) impact collected from respective surveys. A Research Ethics Committee at the University of Chester awarded ethical approval. The study is governed by an Expert Review Group (ERG) consisting of clinicians, allied health professionals, academics, and patient advocacy representatives. These experts provided feedback and recommendations on the study design, including review and approval of all the study materials. In addition, the ERG will be consulted to discuss study results once data is gathered.

Results: The study aims to recruit 170 physicians reporting 1420 eCRFs with an expected return of 527 patient and caregiver surveys. Clinical burden outcomes include symptomatology and comorbidities and motor, cognitive, and behavioral/psychiatric function impact. Economic burden outcomes include direct, indirect, and societal costs, and will be analyzed as per-patient costs over different disease stages. Humanistic burden outcomes include generic and HD-specific QoL measures among patients and caregivers. The ERG reviewed and confirmed the study approach. The main ERG feedback was about patient inclusion and exclusion criteria, stratified sampling on patients, and physicians' recruitment criteria.

Conclusions: The HDBOI study has been initiated and data collection is ongoing. This study will examine the burden of illness of HD in order to strengthen the evidence base of unmet medical needs for the community.

71

Evaluation of the Safety of Deutetrabenazine at Higher Doses to Treat Chorea in Huntington's Disease

Samuel Frank*1 ${ }^{1}$, Christina Vaughan ${ }^{2}$, David Stamler ${ }^{3}$, David Oakes ${ }^{4}$, Matthew D. Davis, Nicholas Gross ${ }^{1}$, Mark Forrest Gordon ${ }^{1}$, Juha-Matti Savola $^{5}$, Maria Wieman ${ }^{1}$, Shirley Eberly ${ }^{4}$, Elise Kayson ${ }^{4}$, Jacquelyn Whaley $^{4}$, Jody Goldstein ${ }^{4}$, and Claudia M. Testa ${ }^{6}$ (on behalf of the Huntington Study Group ARC-HD Investigators)

${ }^{1}$ Teva Pharmaceutical Industries Ltd., Frazer, PA, USA

${ }^{2}$ University of Colorado, Aurora, CO, USA

${ }^{3}$ Alterity Therapeutics, San Francisco, CA, USA

${ }^{4}$ University of Rochester, Rochester, NY, USA

${ }^{5}$ Teva Pharmaceuticals International, Basel, Switzerland

${ }^{6}$ Virginia Commonwealth University, Richmond, VA, USA

Background: In the First-HD pivotal trial, the maximum deutetrabenazine dose evaluated to treat chorea associated with Huntington's disease (HD chorea) was $48 \mathrm{mg} /$ day, which is the approved maximum dose for this population. In ARC-HD, an open-label extension study evaluating the long-term efficacy and safety of deutetrabenazine to treat HD chorea, dosage ranged from to $72 \mathrm{mg} /$ day, with doses $\geq 12 \mathrm{mg} /$ day administered twice daily. Doses in ARC-HD were increased by $6 \mathrm{mg} /$ day per week in a response-driven manner based on efficacy and tolerability until $48 \mathrm{mg} / \mathrm{d}$ (Week 8). At the investigator's discretion, further increases were permitted by $12 \mathrm{mg} /$ day per week to a maximum of $72 \mathrm{mg} /$ day. This post hoc analysis evaluates the safety and tolerability of deutetrabenazine $>48 \mathrm{mg}$ /day compared to $\leq 48 \mathrm{mg} /$ day to treat HD chorea in ARC-HD.

Methods: Patient counts and safety assessments were attributed to patients when they received a dose of either $\leq 48 \mathrm{mg} /$ day or $>48 \mathrm{mg}$ /day. For nine selected adverse events (AEs), we compared AE rates adjusted for duration of drug exposure (as the number of AEs/year) at $\leq 48 \mathrm{mg} /$ day or $>48 \mathrm{mg} /$ day. The AE rates were determined after titration when participants were on stable doses of deutetrabenazine. All subjects provided 
consent to the study in accordance with the informed consent regulations at all involved centers.

Results: All 113 patients were exposed to doses $\leq 48 \mathrm{mg} /$ day (177.1 patient-years) and 49 patients were exposed to doses $>48 \mathrm{mg} /$ day $(74.1$ patient-years). In patients taking deutetrabenazine $>48 \mathrm{mg} /$ day compared to $\leq 48 \mathrm{mg}$ /day after the titration period, there were no apparent differences in exposure-adjusted $\mathrm{AE}$ rates.

Conclusions: Based on clinical experience, some patients with HD may benefit from doses higher than $48 \mathrm{mg}$ /day to adequately control chorea. These doses were tolerated without apparent increase in the exposureadjusted rates of selected AEs after titration. This analysis does not address the occurrence of other AEs or whether adequate efficacy was achieved at lower doses, factors that may have influenced dose increases.

\section{2}

Cerebral Venous Thrombosis, Neutropenia, and Iron Deficiency Anemia in Huntington's Disease: a Case Report with Literature Review

Farinaz Tabibian* ${ }^{1}$, Iman Adibi ${ }^{1}$, Parisa Emami Ardestani ${ }^{1}$, Elnaz Tabibian $^{2}$, Sahar Akbaripour ${ }^{1}$, Katrin Bürk ${ }^{3}$

${ }^{1}$ Ayatollah Kashani Hospital, Isfahan University of Medical Sciences, Isfahan, Iran

${ }^{2}$ Tehran University of Medical Sciences, Tehran, Iran

${ }^{3}$ Kliniken Schmieder Stuttgart-Gerlingen, Gerlingen, Germany

Background: Huntington's disease (HD) has neurologic and nonneurologic manifestations. Although some hematologic disorders were previously seen in HD patients, there is no report of concomitant cerebral venous thrombosis (CVT), iron deficiency anemia (IDA), and neutropenia as HD presentations. We introduce the first case of a young HD patient with these manifestations and review the evidences on their relevance to HD.

Methods: A 30-year-old female was admitted to our clinic for worsening of involuntary movements and imbalance with a past history of CVT and depression. In addition to a comprehensive medical history, we performed neurologic and psychiatric examinations, neuropsychiatric tests, radiologic and para-clinical studies, and genetic testing.

Results: Her clinical syndrome with prominent chorea and psychiatric and cognitive symptoms, as well as a positive family history, suggested HD. The diagnosis was confirmed by genetic testing. Until our first visit, all neurologic and psychiatric abnormalities had been thought to be resulting from the previous CVT. However, no prothrombotic conditions had been found at that time. We also ruled out all common transient and chronic risk factors for development of CVT. Furthermore, we noticed a recurrent mild isolated neutropenia and IDA during her follow-ups. Reviewing the literature, there is experimental evidence for defective blood-brain barrier, increased neurovascular density, and reduced diameter of cerebral vessels in HD. Platelet abnormalities related to thrombus formation have been demonstrated in HD patients and animal models as well. It is not clear whether these changes have direct impact on CVT formation. Despite reports on neutrophil pathology in HD, including increased oxidative damage and mitochondrial abnormalities, neutropenia and its etiological relevance to these findings have not been reported in HD so far. Observation of increased iron stores and coinciding damages in basal ganglia of HD patients, in addition to successful treatment of HD mice with iron-binding compounds, suggest a potential role for iron dysregulation in HD pathogenesis. Only two studies have assessed biochemical serum iron-related measures, and both found no concise explanation for lowered serum ferritin and hemoglobin concentrations in HD.

Conclusions: Experimental evidence suggests that HD vascular and platelet abnormalities may increase thrombogenicity, a phenomenon that could currently be underestimated.
73

Impact of COVID-19 on Patients and Families with Huntington's Disease

DevaKumar Hoskere Sreenivasa

Vikram Hospital, Bangalore, Karnataka, India

Background: The impact of COVID-19 has been multifold across the globe affecting social, family, and personal lives. This impact could be detrimental in people with preexisting chronic illness affecting motor, cognitive, and behavioral aspects.

Objectives: To understand the impact of COVID-19 lockdown in patients and caregivers of Huntington's disease (HD).

Methods: A structured questionnaire-based interview was conducted to understand the effect of "COVID-19 Lockdown" on motor and behavioral activities of daily living in subjects of genetically confirmed HD. Response to change in subjective perception of symptoms in the preceding 3 months (April to June 2020) were collected. Patients who had worsening of any other medical issues in this tenure were excluded. In addition, Zarit burden interview (ZBI) and FACIT-Sp12 scales were collected to understand the caregiver burden and spiritual well-being of patients, respectively.

Results: Twelve HD patients (M:F, 9:3; mean age:53.6 \pm 10.7 years) and their caregivers, who were clinically symptomatic for last $6.1 \pm 3.3$ years completed the interviews. Most were noted to have clinical worsening in relation to motor, behavior, and ADL (58-75\%). Subjective improvement in all factors was noted in one patient. Overall, subjective worsening was noted by six, no change in four, and improvement in two. Personal strain in relationship on ZBI was noted in most, with a score of $35.2 \pm 6.6$ (range: 23-47). In relation to spiritual well-being, the average scores were less than $50 \%$ in relation to the meaning of life, peace, and total scores, indicating overall lower contentment in life.

Conclusions: The effect of COVID-19 lockdown was significant in HD patients and families with most reporting worsening of their motor and behavioral symptoms, indicating the role of external factors on HD. Patients and families with chronic illness require more attention and social support in pandemic outbreaks for reassurance and well-being.

74

Long-Term Efficacy and Safety of Deutetrabenazine for Chorea in Huntington's Disease: Results From the ARC-HD Open-Label Study

Samuel Frank*1, Claudia M. Testa ${ }^{2}$, David Stamler ${ }^{3}$, Elise Kayson ${ }^{4}$, David Oakes ${ }^{4}$, Christina Vaughan ${ }^{5}$, Jody Goldstein ${ }^{4}$, Jacquelyn Whaley $^{4}$, Matthew D. Davis, Mark Forrest Gordon ${ }^{1}$, Juha-Matti Savolay ${ }^{6}$ (on behalf of the Huntington Study Group ARC-HD Investigators)

${ }^{1}$ Teva Pharmaceutical Industries Ltd., Frazer, PA, USA

${ }^{2}$ Virginia Commonwealth University, Richmond, VA, USA

${ }^{3}$ Alterity Therapeutics, San Francisco, CA, USA

${ }^{4}$ University of Rochester, Rochester, NY, USA

${ }^{5}$ University of Colorado, Aurora, CO, USA

${ }^{6}$ Teva Pharmaceuticals International, Basel, Switzerland

Background: Chorea is a prominent motor dysfunction in Huntington's disease (HD). Deutetrabenazine (DTBZ), a VMAT2 inhibitor, is FDAapproved for the treatment of chorea in HD. In the pivotal, 12-week FirstHD trial, DTBZ treatment reduced the Unified Huntington's Disease Rating Scale (UHDRS) total maximal chorea (TMC) score vs. placebo. ARC-HD, an open-label extension study, evaluated long-term safety and 
efficacy of DTBZ dosed in a response-driven manner for treatment of HD chorea.

Methods: Patients who completed First-HD (Rollover) and patients who converted overnight from a stable dose of tetrabenazine (Switch) were included. Safety was assessed over the entire treatment period; exposureadjusted incidence rates (EAIRs; adverse events [AEs] per person-year) were calculated. A stable, posttitration time point of 8 weeks was chosen for efficacy analyses. All subjects provided consent to the study in accordance with the informed consent regulations at all involved centers.

Results: Of 119 patients enrolled (Rollover, $n=82$; Switch, $n=37$ ), 100 (84\%) completed $\geq 1$ year of treatment (mean [SD] follow-up, 119 [48] weeks). End of study EAIRs for patients in the Rollover and Switch cohorts, respectively, were: any AE, 2.6 and 4.3; serious AEs, 0.13 and 0.14; AEs leading to dose suspension, 0.05 and 0.04 . Overall, $68 \%$ and $73 \%$ of patients in Rollover and Switch, respectively, experienced a study drug-related AE. Most common AEs possibly related to study drug were somnolence (17\% Rollover; 27\% Switch), depression (23\%; 19\%), anxiety $(9 \% ; 11 \%)$, insomnia $(10 \% ; 8 \%)$, and akathisia $(9 \% ; 14 \%)$. Rates of AEs of interest include suicidality $(9 \% ; 3 \%)$ and parkinsonism $(6 \%$; $11 \%)$. In both cohorts, mean UHDRS TMC score and total motor score (TMS) decreased from baseline to Week 8; mean (SD) change in TMC score (units) was -4.4 (3.1) and -2.1 (3.3) and change in TMS was -7.1 (7.3) and - 2.4 (8.7) in Rollover and Switch, respectively. While receiving stable dosing from Week 8 to 132 (or end of treatment), patients showed minimal change in the TMC score $(0.9$ [5.0]), but TMS increased compared to Week $8(9.0[11.3])$. Upon drug withdrawal, there were no remarkable AEs and TMC scores increased 4.4 (3.7) units compared to the end of treatment.

Conclusions: The type and severity of AEs observed in long-term DTBZ exposure are consistent with the previous study. Efficacy in reducing chorea persisted over time. There was no unexpected worsening of HD or chorea associated with HD upon DTBZ withdrawal.

75

\section{A Data Analytics-Led Focus on Falls in the Only Huntington's Disease (HD) Inpatient Unit in Ireland}

Muthukumaran Thangaramanujam* ${ }^{*}$, Isabel Chamberlin, Jennifer Hoblyn

Bloomfield Hospital, Trinity College Dublin, Dublin, Ireland (all authors)

Background: A data analytics-driven falls audit was devised and carried out in Bloomfield Hospital, Dublin, Ireland. Every fall that occurs in Bloomfield Hospital has to be reported and documented in the National Incident Management System (NIMS). The nature and circumstances of each person's fall is unique and multifactorial. It was decided to audit data from all falls collectively in Bloomfield Hospital, as this gives thorough insight.

Methods: Fall data from 3 months (quarter) preceding a chosen date were collected and analyzed. The NIMS database system administration was examined in detail over a period of 6 months. Upon extensive research, a quarter-specific, falls audit data-generation tool (form) was successfully created. This query created a generic falls data set for Bloomfield Hospital, and data from 284 falls were captured. These data were then exported as a Comma Separated Value (CSV) file and were refined further with the following NIMS column headings: Record Number, Name, Date, Brief Summary, Severity Rating, Location, Problem / Cause, Outcome, Body Part Injured, and Time of Incident. Detailed analysis was carried out for the whole hospital, and the HD unit's falls data directly informed practice, which led to improved care.

Results: The findings of the Falls Audit 2019 were analyzed in detail by the Multi-Disciplinary Falls Management Committee. The Mode was the particularly interesting statistical descriptor for the HD unit, as it pointed to $50 \%$ of the falls $(n=30)$ in the unit identified to one individual in several locations and times. It highlighted the multi-factorial etiology, such as neuro-psychiatric etiology, mobility status, and environmental causes. As some of these factors could be mitigated, they were actioned which minimized the falls incidences immediately. Currently, the falls mode trend curve has flattened for the unit.

Conclusions: Data analysis of falls that occurred in the HD inpatient unit portrayed several insights, which directly influenced care. As falls would continue to reoccur, particularly in the middle to late stages of $\mathrm{HD}$, it is vital for a data analytics-led falls audit to capture the collective linked HD clinical data associated with every fall. Data mining techniques, such as text analytics and cluster analysis, would be essential for further analysis of such data. This may allow for the development of a hospital-specific predictive analytics tool to focus on providing holistic care to patients with HD.

\section{6}

Postural Control Measures Derived from Wearable IMU Devices in Huntington's Disease: Recommendations for Clinical Outcomes

Radhika Desai* ${ }^{1}$, Miguel Blacutt ${ }^{1}$, Nora E. Fritz ${ }^{2}$, Lisa Muratori ${ }^{3}$, Lori Quinn $^{1}$

${ }^{1}$ Columbia University, New York, NY, USA

${ }^{2}$ Wayne State University, Detroit, MI, USA

${ }^{3}$ Stony Brook University, Stony Brook, NY, USA

Background: Impairments in posture begin in premanifest stages of HD and progress through the course of the disease. However, the components of postural control most vulnerable to HD have yet to be identified. Wearable inertial measurement units (IMUs) provide quantitative measures of balance and gait, yet there are limited data on which measures are best suited to evaluate HD impairments. The aims of this study were to identify 1) differences in postural control between healthy individuals and those with Huntington's disease (HD) from wearable sensors and 2) measures most likely to predict HD diagnosis and clinical ratings of postural control and chorea.

Methods: Forty-one individuals with manifest HD and 14 age-matched healthy controls wore Opal APDM IMUs (lumbar and bilateral feet) during static standing with feet together (FT) and feet apart (FA), and during static sitting. Mean velocity (MV), jerk, root mean squared (RMS), and total sway area (sway) across conditions were provided by APDM (Portland, OR) Mobility Lab software. One-way ANOVAs determined differences between groups in postural control parameters for each task. Random forest analysis identified Feature of Importance of postural variables for HD diagnosis. Stepwise regression determined significant predictors of UHDRS tandem walking and chorea scores.

Results: There were significant differences between HD and healthy for all tasks $(p<.05)$, with the exception of jerk during sitting. Feature of Importance (Gini index) derived by the random forest model revealed jerk FT, jerk FA, sway FA, and RMS FA as measures most important to predict HD diagnosis. Results of the stepwise regression indicated that sway FA and sway sitting predicted UHDRS combined chorea scores $\left(R^{2}=0.4019\right.$, $p<0.001)$. RMS FT, jerk FA, and RMS sitting $\left(R^{2}=0.4162\right.$, $p<0.001)$ significantly predicted UHDRS tandem walking score. Conclusions: Differences in IMU-derived postural outcome measures for standing and sitting tasks indicate an HD-specific impairment to modulate spatial and temporal components of postural stability and sway. Measures of total sway area of FA and sitting are likely influenced by choreic movements, whereas RMS FT, jerk FA, and RMS sitting are likely to best reflect postural control impairments. Ultimately, these results provide insight on IMUderived outcome measures that have potential to serve as biomarkers of postural impairments in HD. 
77

cGAS Promotes the Inflammatory and Autophagy Responses in Huntington's Disease

Manish Sharma*, Sumitha Rajendrarao, Neelam Shahani, Uri Nimrod Ramírez-Jarquín, Srinivasa Subramaniam

Scripps Research Institute, Jupiter, FL, USA (all authors)

Background: Huntington's disease (HD) is caused by an expansion mutation of the N-terminal polyglutamine of huntingtin (mHTT). mHTT is ubiquitously present, but it induces noticeable damage to the brain's striatum, thereby affecting motor, psychiatric, and cognitive functions. The striatal damage and progression of HD are associated with the inflammatory response; however, the underlying molecular mechanisms remain unclear.

Results: Here, we report that cGMP-AMP synthase (cGAS), a DNA sensor, is a critical regulator of inflammatory and autophagy responses in HD. Ribosome profiling revealed that the cGAS mRNA has high ribosome occupancy at exon 1 and codon-specific pauses at positions 171 (CCG) and 172 (CGT) in HD striatal cells. Moreover, the protein levels and activity of cGAS (based on the phosphorylated STING and phosphorylated TBK1 levels), and the expression and ribosome occupancy of cGAS-dependent inflammatory genes ( $\mathrm{Ccl} 5$ and $\mathrm{Cxcl10})$ are increased in HD striatum. Depletion of cGAS diminishes cGAS activity and decreases the expression of inflammatory genes while suppressing the upregulation of autophagy in HD cells. In contrast, reinstating cGAS in cGAS-depleted HD cells activates cGAS activity and promotes inflammatory and autophagy responses. Ribosome profiling also revealed that LC3A and LC3B, the two major autophagy initiators, show altered ribosome occupancy in HD cells. We also detected the presence of numerous micronuclei, which are known to induce cGAS in the cytoplasm of neurons derived from human HD embryonic stem cells.

Conclusions: Collectively, our results indicate that cGAS is upregulated in HD and mediates inflammatory and autophagy responses. Thus, targeting the cGAS pathway may offer therapeutic benefits in HD.

\section{8}

Brain Volume Trajectories in Juvenile-Onset Huntington's Disease

Jordan Schultz , Alison M. Kruger, Vincent A. Magnotta, Timothy R. Koscik, Peg C. Nopoulos

University of Iowa, Iowa City, IA, USA (all authors)

Background: Patients with Juvenile-Onset Huntington's Disease (JoHD) are known to have significant neurodegeneration, but the longitudinal trajectory of change in brain volumes over time has never been investigated.

Objective: To compare longitudinal changes in brain volume of various brain regions between patients with JoHD and patients without the geneexpansion that causes HD (GNE group).

Methods: We evaluated neuroimaging data from JoHD participants from the Kids-JHD study and GNE participants from the Kids-HD study. Participants were included in the JoHD group if they had a total motor score $>20$, a CAG $<36$, and had received a clinical diagnosis of JoHD from a neurologist. Participants were included in the GNE group if they had neuroimaging data available and a $\mathrm{CAG}<36$. We performed linear mixed-effects regression analyses to compare the longitudinal changes in volume of the whole brain, cerebrum, cerebral cortex, cerebral white matter, caudate, putamen, thalamus, globus pallidus, hippocampus, amygdala, and cerebellum between the JoHD and GNE groups. We evaluated each brain region without correcting for ICV or whole brain volume and again after controlling for whole brain volume. Models controlled age, sex, and parental socioeconomic status and included random effects per participant and family.

Results: The patients with JoHD had significantly faster rates of decline in their volumes of whole brain, cerebrum, cerebral cortex, caudate, putamen, globus pallidus, thalamus, and amygdala relative to the GNE group. Earlier in life, the JoHD group had larger volumes of their whole brain, cerebrum, cerebral cortex, and thalamus relative to the GNE group. The JoHD group demonstrated significant proportional enlargement (relative to whole brain) of the hippocampus and cerebellum over time compared to the GNE group.

Conclusions: Subcortical structures, such as the caudate and putamen, appear to be significantly degenerated very early in life in JoHD but were still degenerating significantly faster compared to the GNE group. The thalamus, cerebrum, cerebral cortex, and whole brain were enlarged earlier in life relative to the GNE group but had significantly faster rates of decline over time. The cerebellum appears to be spared from neurodegeneration in JoHD and even becomes proportionally enlarger over time. These results provide additional information regarding the underlying neuropathology of JoHD.

\section{9}

\section{Development of Assessments for Later Stage Huntington's Disease: UHDRS Structured Interview of Function and HD Clinical Status Questionnaire}

Matthew W. Roche*1 ${ }^{1}$, O.J. Handley ${ }^{2}$, S. Capodarca ${ }^{3}$, S. Frank ${ }^{4}$, R.L.M. Fuller $^{1}$, M. Orth ${ }^{5}$, J. Levey ${ }^{1}$, E. Neacy ${ }^{1}$, C. Sampaio ${ }^{1}$

${ }^{1}$ CHDI Foundation, Inc., New York, NY, USA

${ }^{2}$ Cardiff University, Cardiff, UK

${ }^{3}$ European Huntington's Disease Network, Florence, Italy

${ }^{4}$ Boston University School of Medicine, Boston, MA, USA

${ }^{5}$ University of Ulm, Ulm, Germany

Background: This study aims to provide preliminary clinimetric properties for such measures: the UHDRS Structured Interview of Function (UHDRS SIF) and HD Clinical Status Questionnaire (HDCSQ). Both assessments are administered to a companion either in-person or remotely (i.e., by phone contact with the companion), and the properties of these tests will be evaluated in a two-part study using the methods of Classical Test Theory (CTT) and Item Response Theory (IRT).

Objective: To obtain estimates for the clinimetric properties of the UHDRS SIF and HDCSQ.

Methods: Up to 170 dyads of Manifest HD Gene Expansion Carrier (HDGEC) Participants and their Companion Participants are planned to be enrolled in this study from approximately 20 English-speaking study sites. The study includes two sequential parts. In Part 1, we will use the methods of CTT to evaluate the UHDRS SIF, a structured interview designed to gather information for making ratings on the UHDRS ' 99 functional scales (TFC, FAS and IS). In Part 2, we will use the methods of CTT and IRT to assess the clinimetric properties of the HDCSQ, a questionnaire designed specifically to capture information on disease milestones that occur during the later stages of HD, and the UHDRS SIF. In both parts, Companion Participants will complete a Companion Information Form, a short questionnaire asking about the Companion Participant's perceptions and experiences as a caregiver/companion to the Manifest HDGEC Participant. Recruitment is scheduled to run from 4Q2020 until 2022. Preliminary results from Part 1 will be available during 2021 and a full report will be available in 2022. Upon establishing the clinimetric properties of the scales, these assessments may be used for planning studies or incorporated into observational and interventional studies of HD. 
Conclusions: Including a more advanced patient population will empower them to participate and will promote their valued contribution to research.

80

A Potent and Short-Lived Biologic CRISPR-RNP Permanently Lowers Mutant Huntingtin Protein in Adult Animals in One-Time Treatment

Brett Staahl ${ }^{1}$, Jennifer K. Sabo* ${ }^{1}$, Madhurima Benekareddy ${ }^{2}$, Cole Urnes $^{3}$, Talia Wenger ${ }^{4}$, Gabriela Acevedo Munares ${ }^{1}$, Katherine E. White, Alex Hao ${ }^{1}$, Anirvan Ghosh ${ }^{5}$, Jennifer A. Doudna ${ }^{1}$

${ }^{1}$ University of California, Berkeley, Berkeley, CA, USA

${ }^{2}$ F. Hoffmann-La Roche, Ltd., Basel, Switzerland

${ }^{3}$ University of Southern California, Los Angeles, CA, USA

${ }^{4}$ University of California, San Francisco, San Francisco, CA, USA

${ }^{5}$ Unity Biotechnology, South San Francisco, CA, USA

Background: Gene-editing technology targeted at the underlying cause of a genetically defined disease has the potential to halt the progression of the disease after a single administration.

Methods: Here, we address this potential in the context of Huntington's disease (HD) by using a novel cell-penetrating Cas9 RNP (4xNLS-RNP) that is programmed to inactivate the mutant Huntingtin (mHTT) gene. Using convection-enhanced delivery, we targeted neurons in the striatum and motor cortex, the circuit most affected in HD.

Results: We show that permanent inactivation of mHTT in neurons leads to a decrease in levels of mHTT protein and positively alters the motor coordination deficit in HD mice.

Conclusions: This study paves the way for further investigation of a potent short-lived, cell-penetrating, biologic Cas9 RNP for therapeutic use in neurodegenerative diseases.

81

Functional Changes in the Central Autonomic Network Contributes to Autonomic Dysfunction in Premanifest HD

Jordan Schultz* ${ }^{1}$, Timothy R. Koscik ${ }^{1}$, Vincent A. Magnotta ${ }^{1}$, Alivia N. Brinker $^{2}$, Amanda Heinzerling ${ }^{1}$, John A. Kamholz ${ }^{1}$, Peg C. Nopoulos ${ }^{1}$

${ }^{1}$ University of Iowa, Iowa City, IA, USA

${ }^{2}$ University of Missouri-St. Louis, St. Louis, MO, USA

Background: Patients with Huntington's disease (HD) have been found to have dysregulations of their autonomic nervous systems (ANS), with a predominance of sympathetic tone. Clinical manifestations consistent with ANS dysfunction occur in the premanifest phase of the disease.

Objective: The aim of this pilot study was to assess functional connectivity of central autonomic network (CAN) in premanifest patients with HD.

Methods: We recruited 10 male subjects with a known $\mathrm{CAG} \geq 36$ to the University of Iowa. To be eligible for this study, participants had to have a diagnostic confidence level of $<4$ at the time of their visit. We recruited nine male control subjects with no family history of HD. All participants underwent a resting-state, functional MRI study and an electrocardiogram to perform heart rate variability analyses. A single seed was placed in the right anterior insula (rAI) and a region-of-interest (ROI)-to-ROI analysis was conducted within brain regions of the CAN. Fisher's R-to-Z transformations were performed to quantify functional connectivity between brain regions. ANCOVA analyses were performed to quantify differences in functional connectivity between the HD group and the controls, controlling for age and BMI. Functional connectivity measures were directly correlated to measures of heart rate variability.

Results: Participants in the HD group had significantly decreased functional connectivity relative to the control group between the rAI and (1) right ( $p=0.03)$ and left $(p=0.009)$ rostral prefrontal cortex (RPFC), (2) left anterior insula $(p=0.012)$, and (3) anterior cingulate cortex (ACC) $(p=0.027)$. They also had significant decreases in the root mean square of successive RR interval differences (RMSSD) $(p=0.037)$ and the percentage of successive RR intervals that differ by more than 50 milliseconds (pNN50) $(p=0.044)$ compared to the control group. Lower values are indicative of decreased parasympathetic tone. There were significant correlations with pNN50 values and functional connectivity between the rAI and right and left RPFC ( $p=0.039$ and $p=0.011$, respectively).

Conclusions: To the best of our knowledge, this is the first time that alterations in the functional connectivity of the CAN have been reported in premanifest HD. This pilot suggests that changes in functional connectivity within the CAN may mediate dysfunction of the ANS seen in HD.

82

A Qualitative Examination of Apathy and Physical Activity in Huntington's Disease

Ciaran Friel ${ }^{1}$, Sophie Andrews ${ }^{2}$, Trevor T-J Chong ${ }^{3}$, Julie Stout ${ }^{3}$, Lori Quinn* ${ }^{4}$

${ }^{1}$ Northwell Health, New Hyde Park, NY, USA

${ }^{2}$ NeuRA, Randwick, Australia

${ }^{3}$ Monash University, Melbourne, Australia

${ }^{4}$ Columbia University, New York, NY, USA

Background: Regular physical activity is an essential component of physical and mental health and is especially important in motor diseases like Huntington's disease (HD). Individuals with HD display a complex array of motor, cognitive, and behavioral symptoms that can interfere with physical activity engagement, including apathy, defined as quantitative reduction in self-generated or purposeful behavior. Apathy is associated with reduced treatment seeking and is often cited as a barrier to participation in physical activity interventions. Current frameworks of apathy emphasize its multidimensional presentation with differential effects on behavior. How these dimensions manifest and affect physical activity in HD, however, has not been studied, and may be best captured using qualitative methods to understand people's lived experiences.

Methods: We used a qualitative approach to examine the subjective experience of apathy and its impact on physical activity. Adopting a purposive sampling approach, we sampled 10 people with early-stage manifest HD. We conducted interviews using a preplanned question schedule, alongside a structured clinicianrated apathy interview (Lille Apathy Rating Scale). To facilitate characterization of the sample, participants completed self-report measures of fatigue, self-efficacy, depression, and physical activity behavior.

Results: Four related sub-themes emerged: (1) apathy leads to difficulties in initiation in HD, (2) people with HD have difficulties identifying goals reflecting cognitive apathy, (3) fatigue impacts physical activity and overlaps conceptually with apathy, and (4) prompts, routine, and structure promote physical activity and need to be implemented early in the disease course.

Conclusions: We observed overlap in the conceptualization of apathy and fatigue, and further work is needed to dissociate these syndromes. In combination, our findings suggest apathy is not unidimensional and that physical activity interventions must be tailored to the multiple ways that apathy may present in people with HD. 
83

Exploring Factors Associated with Physical Fitness in People with Huntington's Disease: Results from the PACE-HD Baseline Analysis

Lori Quinn*1 ${ }^{1}$, Katie Taiyari ${ }^{2}$, Rebecca Playle ${ }^{2}$, Rhys Williams-Thomas ${ }^{2}$, Cheney Drew ${ }^{2}$, Beth Ann Griffin ${ }^{3}$, Anne Rosser ${ }^{2}$, Monica Busse ${ }^{2}$

${ }^{1}$ Columbia University, New York, NY, USA

${ }^{2}$ Cardiff University, Cardiff, UK

${ }^{3}$ RAND Corporation

Background: Regular physical activity and exercise are emerging as important interventions for the ongoing management of Huntington's disease (HD). This study aimed to evaluate modifiable and nonmodifiable factors associated with physical fitness to inform development of personalized exercise approaches.

Methods: Patients with early-mid stage HD participating in the PACEHD study at baseline formed the population for these analyses. Participants completed the PACE-HD battery of assessments (predicted VO2 max using cycle ergometry, self-reported and quantitative measures of physical activity, HD-PRO-TRIAD, and walking endurance). Linked data from Enroll-HD (CAG, motor, cognitive, behavioral, and quality of life) were accessed via a specific data request. We classified pharmacotherapy by indication and, specifically, drugs prescribed for HDassociated symptoms. We used the prognostic index normed for HD (PINHD) to provide a measure of current HD status. Prespecified univariable and multivariable regression models were derived to assess associations between data. A list of potentially important variables was selected based on findings from the literature. Model diagnostics were used to assess model fit and that the statistical assumptions of the linear regression were satisfied.

Results: Significant relationships were identified between fitness and measures of HD symptom severity in univariate modeling. In initial multivariate modeling, those taking medication for movement disorders had lower fitness levels. Disease status (PINHD) was not associated with fitness in the multivariable model. Subsequent modeling, where PINHD was replaced by its components (CAG, TMS, and SDMT), demonstrated that CAG repeat length, rather than TMS and SDMT, was associated with fitness in HD.

Conclusions: Medication prescribed for movement disorders should be considered when planning and evaluating exercise interventions. CAG expansion has an established relationship with motor onset and progression in HD; yet, clinically assessed cognitive and motor status were not associated with fitness in HD in our dataset. This is a finding warranting further investigation.

\section{4}

\section{Executive Functioning Changes in Early Huntington's Disease: a Qualitative Research Study}

Jennifer Petrillo Billet*1 ${ }^{1}$, Adele Levine ${ }^{2}$, Jessica Markowitz ${ }^{2}$, Jason Johannesen $^{3}$, Teya Lovell ${ }^{2}$, Alissa Rams ${ }^{2}$, Emily Gusse ${ }^{1}$, Stefan Cano ${ }^{2}$, Aaron Koenig ${ }^{1}$, James J. Doherty ${ }^{2}$, Stephen J. Kanes ${ }^{1}$

${ }^{1}$ Sage Therapeutics, Cambridge, MA, USA

${ }^{2}$ Modus Outcomes

${ }^{3}$ Yale School of Medicine, West Haven, CT, USA

Background: Huntington's disease (HD) is a rare, genetic, neurodegenerative disease characterized by progressive motor, cognitive, and behavioral impairments. The early stage involves cognitive changes, including difficulty with multitasking, organizing thoughts, planning, and decisionmaking. During this period, patients often begin to experience challenges in their day-to-day lives. However, to date, there is little research to understand these challenges from the patients' perspectives.

Objective: To understand how individuals with early HD describe cognitive changes and the impact these have on daily functioning, using qualitative research methods.

Methods: Two advocacy agencies (HDYO, HDSA) assisted with recruiting gene-positive (CAG repeats $\geq 36$ ), early-stage HD patients, 25-65 years old, with self-reported cognitive symptoms and nearnormal daily functioning. Participants described cognitive symptoms and their impact on daily life in qualitative interviews. Semi-structured concept elicitation interviews were conducted, and transcripts were analyzed thematically through line-by-line open and inductive coding for concepts important to patients. Summary statistics were generated. Participants gave their consent. The study was approved by a central IRB. Results: Participants included 19 patients and 10 patient/care partner dyads. Patients were 40 (6) (mean [SD]) years old, 61\% female, 100\% non-Hispanic white, with a family history of HD, HD diagnosed 19 years prior, and CAG repeats ranging from 36 to 48 . Care partners were 42 (7) years old, $50 \%$ female, $80 \%$ non-Hispanic white, and $70 \%$ spouses. Both patients and caregivers reported cognitive changes across all aspects of executive functioning, including adapting and flexibility, processing information, planning and problem-solving, multitasking, and selective attention. Functional impacts on instrumental activities of the patients' daily living included work (meeting deadlines or priority changes), driving (getting lost, forgetting to put in the park), managing finances (making errors when paying bills), and household activities (initiating chores, difficulty cooking more than one thing).

Conclusions: Interviews revealed a decline in higher-order functional skills associated with cognitive change early in HD. There is a need for further research to adequately quantify the impact cognitive symptoms have on functioning in the population with early HD-especially in the workplace, at home, and while driving - to better support patient needs.

\section{5}

Subcortical T1- $\rho$ MRI Abnormalities in Juvenile Onset Huntington's Disease

Jordan Schultz*1, Alexander Tereshchenko ${ }^{1}$, Ansley Kunnath ${ }^{2}$, Joel A. Bruss $^{1}$, Vincent Magnotta ${ }^{1}$, Peg C. Nopoulos ${ }^{1}$

${ }^{1}$ University of Iowa, Iowa City, IA, USA

${ }^{2}$ Vanderbilt University, Nashville, TN, USA

Background: T1-rho (T1 $\rho)$ is a novel neuroimaging technique that has been shown to be able to assess metabolic abnormalities in Huntington's disease (HD). No reports of the use of T1 $\rho$ in patients with juvenile-onset HD exist in the literature.

Objective: To compare subcortical metabolic abnormalities between patients with JoHD and patients without the gene expansion that causes HD (GNE group) using T1-Rho (T1 $\rho$ ) MRI. We also investigated relationships between $\mathrm{T} 1 \rho$ relaxation times and clinical measures in the JoHD group.

Methods: T1 $\rho$ neuroimaging was used to compare brain regions of 11 JoHD participants (13 neuroimaging studies) and 38 controls (39 neuroimaging studies) from the Kids-JHD study. Increased T1 $\rho$ relaxation times are indicative of metabolic abnormalities often associated with neuronal damage. Region-of-interest analyses were used to assess differences in quantitative T1 $\rho$ relaxation times between the JoHD. We used linear mixed-effects analyses to compare $\mathrm{T} 1 \rho$ relaxation times between the JoHD and GNE groups, controlling for age and sex and a random effect per participant. We then performed linear mixed models in the JoHD group only to investigation relationships between $\mathrm{T} 1 \rho$ relaxation times 
and disease burden scores and total motor scores that controlled for age and $\mathrm{CAG}$ repeat length.

Results: We found that mean relaxation times in the caudate $(p<0.001)$, putamen $(\mathrm{p}<0.001)$, globus pallidus $(\mathrm{p}<0.001)$, and thalamus $(p<0.001)$ were increased in JoHD participants compared to controls. We found significant positive relationships between CAG repeat length and T1 $\rho$ relaxation times in the caudate $(p=0.018)$, putamen $(p=0.006)$, globus pallidus $(p<0.0001)$, and thalamus $(p=0.026)$. Disease burden scores significantly predicted T1 $\rho$ relaxation times in the caudate $(p=0.003)$ and the thalamus $(p=0.012)$. Lastly, we found that higher mean $\mathrm{T} 1 \rho$ relaxation times in the caudate $(\mathrm{p}=0.006)$, putamen $(p=0.011)$, globus pallidus $(p=0.008)$, and thalamus $(p=0.042)$ were positively related to increased UHDRS scores among the JoHD participants. Conclusions: T1 $\rho$ MRI may be a valuable biomarker for monitoring disease progression and evaluating future clinical trials in JoHD. T1 $\rho$ MRI abnormalities in JoHD participants were found within the caudate, putamen, globus pallidus, and thalamus, and mean $\mathrm{T} 1 \rho$ relaxation times within these regions were predictive of disease severity and motor deficits.

\section{6}

Understanding the Role of Biological Aging in Huntington's Disease

Emily Machiela* ${ }^{1}$, Shagun Metha, Ritika Jeloka, Mandi Schmidt, Virginia Mattis ${ }^{2}$, Amber Southwell ${ }^{1}$

${ }^{1}$ University of Central Florida, Orlando, FL, USA

${ }^{2}$ FUJIFILM Cellular Dynamics, Madison, WI, USA

Background: Although the mutation that causes Huntington's disease (HD) is present from birth, most patients can live several decades without overt symptoms, begging the question of how the biological aging process may contribute to the onset of disease. Biological aging is defined by nine hallmarks. Interestingly, most of these hallmarks are also implicated in HD pathogenesis. Furthermore, accelerated markers of aging are found in HD brains, further supporting the hypothesis that aging contributes to HD pathology.

Methods: To investigate the role of biological aging in $\mathrm{HD}$, we are using primary neurons from HD mice and wildtype littermates, as well as induced pluripotent stem cell (iPSC)-derived neurons from HD patients and healthy controls. Both primary neurons and iPSC-derived adult-onset HD neurons are embryonic and display no spontaneous neurodegeneration. To overcome this, we treated HD and control cells with progerin, a truncated protein created by alternative splicing of the LMNA gene. Accumulation of progerin causes Hutchinson-Gilford progeria, a disease of rapid aging. Progerin has also been found in healthy tissues during the aging process and has been linked to cellular senescence. In doing this, we can compare cellular phenotypes in HD neurons that are physiologically "young" and "old," but chronologically identical, to determine the role of physiologic aging in the cellular toxicity of HD.

Results: We found that aging neurons with progerin altered protein markers associated with age, caused nuclear blebbing, and decreased dendritic length irrespective of genotype. Interestingly, progerin also selectively increased DNA damage and decreased stress resistance specifically in HD neurons.

Conclusions: This suggests that aging neurons may uncover phenotypes relevant to HD pathology. Ultimately, this work will shed light on aging-associated factors that contribute to HD pathogenesis and whether antiaging treatments may be beneficial for HD patients.
87

Abnormal Cerebellar Circuitry in Juvenile-Onset Huntington's Disease

Jordan Schultz, Peg C. Nopoulos

University of Iowa, Iowa City, IA, USA (all authors)

Background: The cerebellum has been shown to be proportionally enlarged relative to the whole brain in patients with Juvenile-Onset Huntington's Disease (JoHD). It has been hypothesized that the cerebellum may play a compensatory role in HD, but functional MRI studies have never been employed in JoHD to investigate cerebellar circuits that may contribute to compensatory actions.

Objective: We aimed to identify cerebellar circuits that differed significantly from participants without the gene expansion that causes HD (GNE group) that may explain some of the unique symptoms of JoHD.

Methods: We evaluated resting-state functional MRI (fMRI) data from JoHD participants from the Kids-JHD study and GNE participants from the Kids-HD study. Participants were included in the JoHD group if they had a total motor score $\geq 20$, a $C A G \geq 36$, and had received a clinical diagnosis of JoHD from a neurologist. For participants with multiple fMRI studies, we evaluated their first available study. We placed seeds in the anterior cerebellum and the posterior cerebellum and performed whole-brain region-of-interest connectivity analyses. Fisher R-to-Z transformations were performed to quantify functional connectivity between regions. ANCOVA models then compared mean Z-scores between groups, while controlling for age and sex.

Results: There were eight participants with JoHD that had fMRI data available at their first visit, and 71 GNE participants. The JoHD group had significantly decreased functional connectivity between the anterior cerebellum and the right and left caudate. Additionally, the JoHD group demonstrated increased connectivity between the anterior cerebellum and the motor cortex compared to the GNE group. The JoHD group also had decreased connectivity between the posterior cerebellum and left caudate compared to the GNE group. The JoHD also showed hyper-connectivity relative to the GNE group between the posterior cerebellum and areas of the motor cortex.

Conclusions: Patients with JoHD showed decreased functional connectivity between the cerebellum and the caudate, which may be predicted given the significant neurodegeneration in the striatum. However, the increased functional connectivity observed in the JoHD between the cerebellum and the motor cortex is of particular interest and may be indicative of cerebellar compensatory mechanisms in JoHD. Further investigation is necessary.

\section{8}

The PROOF-HD Study: Pridopidine's Outcome on Function in Huntington's Disease

Ralf Reilmann ${ }^{1}$, Andrew S. Feigin ${ }^{2}$, Michal Geva*3, Andrew McGarry ${ }^{4}$, Yael Cohen ${ }^{3}$, Noga Gershoni-Emek ${ }^{5}$, Munish Mehra ${ }^{3}$, C. Warren Olanow $^{6}$, Karl Kieburtz ${ }^{7}$, Michael R. Hayden ${ }^{8}$

${ }^{1}$ George Huntington Institute, Münster, Germany

${ }^{2}$ New York University, New York, NY, USA

${ }^{3}$ Prilenia Therapeutics, Ltd, Herzliya, Israel

${ }^{4}$ Cooper University Hospital, Cherry Hill, NJ, USA

${ }^{5}$ Tel Aviv University, Tel Aviv, Israel

${ }^{6}$ Mount Sinai School of Medicine, New York, NY, USA

${ }^{7}$ University of Rochester, Rochester, NY, USA

${ }^{8}$ University of British Columbia, Vancouver, British Columbia, Canada 
Background: Pridopidine is a well-tolerated oral drug, with potent agonist activity at the Sigma-1 Receptor (S1R). The S1R is located at the ERmitochondria interface, where it regulates diverse cellular processes, such as $\mathrm{Ca}^{2+}$ signaling, the ER stress response, and mitochondrial function. To date, other long-term ( $\geq 1$ year) clinical trials in HD have not shown benefit on the rate of decline in TFC. In the PRIDE-HD trial, post hoc analysis shows maintenance of TFC at week 52 in Stage 1 and 2 HD participants treated with $45 \mathrm{mg}$ bid pridopidine vs placebo (1.16, nominal $p=0.0003$ ). This effect remained significant using the conservative multiple imputation with missing not at random analysis (MNAR) (nominal $p=0.016$ ). Responder analysis showed that $45 \mathrm{mg}$ bid reduced the probability of TFC decline by $80 \%$ (nominal $p=0.02$, week 52 ). Exploratory post hoc analysis showed improved cUHDRS (combined TMS, TFC and SDTM scores) (nominal $p=0.04$ ). Q-motor, used as an exploratory endpoint in PRIDE-HD, demonstrated improvement in the finger tapping inter-onset interval at weeks 26 (nominal $p=0.02$ ) and 52 (nominal $p=$ 0.195 ). Human PET imaging previously showed selective and robust S1R occupancy by pridopidine at this dosage.

Objective: To evaluate the effect of pridopidine $45 \mathrm{mg}$ bid vs. placebo on TFC in early HD patients.

Methods: PROOF-HD is a 65 -week, double-blind, placebo-controlled, phase 3 trial. Design is based on data from prior studies and reflects additional recent insight about pridopidine's mechanism of action. The target patient population is early $\mathrm{HD}(\mathrm{TFC} \geq 7)$ patients with an expected TFC decline of -0.8 to -1 points/year. Analysis of ENROLL-HD data suggests that additional inclusion criteria of UHDRS-IS $\leq 90 \%$ and TMS $\geq 20$ enriches enrollment of participants already showing some functional decline. The primary endpoint is the mean change from baseline to week 65 in TFC. The secondary endpoints are the proportion of patients with no TFC decline (TFC $\geq 0$ ) at week 65 , the change from baseline to week 65 in Total Motor Score (TMS), the composite UHDRS (cUHDRS), and Qmotor testing.

Results: Pridopidine improved TMS in the earlier and shorter 12-week HART (nominal $p=0.04$ ) and 26-week MermaiHD $(p=0.004)$ trials.

Conclusions: PRIDE-HD demonstrated a potential benefit of pridopidine on maintenance of TFC. PROOF-HD aims to further evaluate and assess this effect in an optimized early participant cohort, and to examine motor effects.

\section{9}

Remote Monitoring of Physical Activity, Falls, and Near-Falls in Huntington's Disease Using a Wearable Sensor Platform

Jamie Adams*1, Ray Dorsey ${ }^{1}$, Emma Waddell ${ }^{1}$, Madeleine Coffey ${ }^{1}$, Christopher Tarolli ${ }^{1}$, Ruth Schneider ${ }^{1}$, Ana Enriquez ${ }^{2}$, Ashkan Vaziri ${ }^{2}$, Hung Nguyen ${ }^{2}$

${ }^{1}$ University of Rochester, Rochester, NY, USA

${ }^{2}$ BioSensics LLC, Boston, MA, USA

Background: Increased physical activity has been shown to improve motor function in individuals with Huntington's disease (HD). Furthermore, falls are common in patients with HD, and the social and economic impact of falls can be devasting. Wearable sensors can be used to remotely monitor physical activity and detect falls, which can provide healthcare providers with continuous insight on the disease progression and potential impact of treatments. The aim of this study is to investigate the use of a wearable sensor-based system to remotely monitor physical activities, falls, and near-falls in patients with HD.

Methods: Forty-four participants were consented and enrolled in the study and 41 (mean age $=49.8 \pm 14.4$ years old) were included in the analysis (three were excluded due to missing data). The study was approved by the University of Rochester. Eight participants were classified as prodromal HD, 21 as manifest HD, and 12 were controls. Participants were asked to visit the clinic every 3 months for up to 18 months. During each visit, patients were given an accelerometer-based wearable sensor (PAMSys, BioSensics, Newton MA) and a tablet for the collection of patient-reported outcomes for 1 week after each visit. In addition, nearfalls were reported by the patient using the study tablet. Univariate analysis was used to compare across groups, and Cohen's effect size (d) was calculated adjusting for gender, age, and BMI.

Results: During the remote monitoring period, a total of 43 falls occurred across all participants. HD participants experienced more falls per week than controls $(0.608$ vs. 0.031 falls/week, $p=0.107, \mathrm{~d}=0.60)$ and prodromal HD ( 0.324 falls/week, $p=0.559, \mathrm{~d}=0.26)$. In addition, HD participants also reported more near falls $(n=9)$ than prodromal $\operatorname{HD}(n=2)$ and control $(n=1)$. Results also showed HD participants showed comparable physical activity level to controls (7415 vs. 7354 steps/day, $p=$ 0.963 ). In addition, patients were highly compliant in wearing the sensors during the study period ( $94 \%$ compliance rate, compliance is defined as wearing the sensor for at least $18 \mathrm{~h}$ during each day).

Conclusions: We developed a wearable sensor platform that can be used to remotely monitor falls and physical activity in patients with HD. Furthermore, the platform is capable of capturing near-falls and other symptoms which can provide comprehensive longitudinal assessment of the state of the disease.

\section{0}

\section{Characteristics and Treatment Patterns in Patients with Huntington} Disease: Current Data from Enroll-HD

Erin E. Furr-Stimming ${ }^{1}$, Daniel O. Claassen ${ }^{2}$, Mallory Farrar*3 ${ }^{3}$, Deepika Jethwani $^{3}$, Tara Carmack ${ }^{3}$, Chuck Yonan ${ }^{3}$, Dietrich Haubenberger ${ }^{3}$

${ }^{1}$ University of Texas, Houston, TX, USA

${ }^{2}$ Vanderbilt University, Nashville, TN, USA

${ }^{3}$ Neurocrine Biosciences, Inc., San Diego, CA, USA

Background: Enroll-HD is a worldwide, prospective, observational study for individuals who have (or are at-risk) for Huntington's disease (HD). Enroll-HD data were analyzed to determine treatment patterns for chorea in North America based on different stages of functional severity. Methods: Participants $\geq 18$ years of age, were categorized as premanifest or manifest HD, based on clinician assessment. Clinical data were defined, specifically using the Unified Huntington's Disease Rating Scale (total motor scale [TMS] score, total maximal chorea [TMC] score, total functional capacity [TFC], diagnostic confidence level [DCL]), sociodemographic characteristics, medications, and indicated use. Data were analyzed descriptively; mean values are presented with standard deviations $( \pm \mathrm{SD})$.

Results: Of 3527 patients included for analysis, 1283 were categorized as premanifest and 2244 were categorized as manifest (motor manifest or manifest with any HD symptom). 91.1\% (2045/2244) of manifest patients had a DCL of 4 ("motor abnormalities that are unequivocal signs of HD"); most were in TFC stage I $(n=754)$, II $(n=915)$, III $(n=462)$, or IV $(n=93)$. Sociodemographic characteristics of manifest patients included: mean age, $52.4 \pm 12.8$ years; female, $52.0 \%$; white, $89.5 \%$; married, $56.3 \%$. Based on a score $\geq 2$ ("mild common" or "moderate intermittent") in any TMC body region, chorea was evident in $66.2 \%$ $(1485 / 2244)$ of manifest patients (TFC stage I, 57.6\%; TFC stage IV, $79.6 \%$ ). Mean TMS and TMC scores in manifest patients were $34.3 \pm$ 17.8 and $8.7 \pm 5.0$, respectively; mean TMC scores ranged from $7.6 \pm 4.4$ (TFC stage I) to $10.5 \pm 5.5$ (TFC stage IV). $33.9 \%$ (761/2244) of manifest patients had chorea and were prescribed a medication for chorea. Among these 761 patients, the most commonly reported chorea medications were tetrabenazine $(51.1 \%)$, risperidone $(22.5 \%)$, and amantadine $(12.1 \%)$. $38.7 \%(322 / 831)$ of manifest patients who were prescribed an antipsychotic for any reason received an antipsychotic indicated for chorea. 
Conclusions: Our results demonstrate, as previously described, that chorea severity increases with more advanced stages of disease. Interestingly, while $66.2 \%(1485 / 2244)$ of patients with manifest HD had chorea, only $33.9 \%$ (761/2244) were prescribed treatments for chorea. Results are limited by the inherent constraints of a real-world database. Continued research is needed to better understand treatment patterns for chorea in patients with HD.

\section{1}

\section{Does the Composite Unified Huntington's Disease Rating Scale (cUHDRS) Correlate with Disease Progression in Huntington's Disease?}

Haileigh Smith

University of California, San Diego, La Jolla, CA, USA

Background: The cUHDRS is a composite endpoint developed to evaluate disease progression in early-to-moderate manifest HD. It comprises four assessments: the UHDRS Total Functional Capacity (TFC), the UHDRS Total Motor Score (TMS), Symbol Digit Modalities Test (SDMT), and Stroop Word Reading (SWR).

Objective: To examine the usefulness of the cUHDRS in reflecting disease progression in a well-characterized group of mild to moderate Huntington's disease (HD) subjects followed at one academic HD center. Methods: We used Pearson product-moment correlation to examine the association between cUHDRS scores and various UHDRS and nonUHDRS clinical measures in 84 subjects with mild-moderate HD. The clinical measures included the TFC; the TMS; the UHDRS Independence Scale (IS); SDMT; SWR; Mini-Mental State Examination (MMSE); Montreal Cognitive Assessment (MoCA); the Computerized Test of Information Processing (CTiP), which comprises three reaction-time subtests that successively increase in task complexity; Total Body Sway (TBS), as captured by a computerized balance assessment device (BTrackSTM); and total score on the Huntington's Disease Behavioral Questionnaire (HD-BQ).

Results: We found that, not surprisingly, there were very strong correlations between the cUHDRS and assessments on which it is based, including the TFC $(r=0.815)$, TMS $(r=-0.872), \operatorname{SDM}(r=0.860)$, and SWRT $(r=0.812)$. For other cognitive measures, however, such as the MMSE $(r=0.593)$, MoCA $(r=0.610)$, CTiP Choice Reaction Time $(r=-$ $0.540)$, and CTiP Semantic Search Reaction Time $(r=-0.661)$, correlations were less robust. Correlations between the cUHDRS and another assessment of daily functioning, the UHDRS Independence Scale, were strong $(r=0.777)$. There was a strong correlation with balance as appraised by TBS $(r=-0.623)$ but a poor correlation with behavior as assessed by the HD-BQ $(r=-0.229)$.

Conclusions: The cUHDRS appears to reflect clinical progression reasonably well, particularly with regard to measures on which it is based. However, apart from behavioral evaluations, it also correlates well with independent assessments, which provides support for its usefulness as a primary outcome measure in clinical trials of mild to moderate HD.

\section{2}

\section{Estimating Disease Onset from Change Points of Markers Measured with Error}

Tanya Garcia*1, Unkyung Lee ${ }^{1}$, Raymond J. Carroll ${ }^{1}$, Yuanjia Wang ${ }^{2}$, Karen Marder $^{2}$

${ }^{1}$ Texas A\&M University, College Station, TX, USA

${ }^{2}$ Columbia University, New York, NY, USA
Huntington's disease (HD) is an autosomal, dominant, neurodegenerative disease without clearly identified biomarkers for when motor-onset occurs. Current standards to determine motor-onset rely on a clinician's subjective judgment that a patient's extrapyramidal signs are unequivocally associated with HD. This subjectivity can lead to error, which could be overcome using an objective, data-driven metric that determines motor-onset. Recent studies of motor-sign decline - the longitudinal degeneration of motor-ability in patients - have revealed that motor-onset is closely related to an inflection point in its longitudinal trajectory. We propose a nonlinear location-shift marker model that captures this motor-sign decline and assesses how its inflection point is linked to other markers of HD progression. We propose two estimating procedures to estimate this model and its inflection point: one is a parametric method using a nonlinear mixed-effects model and the other is a multi-stage nonparametric approach, which we developed. In an empirical study, the parametric approach was sensitive to correct specification of the mean structure of the longitudinal data. In contrast, our multi-stage nonparametric procedure consistently produced unbiased estimates regardless of the true mean structure. Applying our multi-stage nonparametric estimator to PREDICT-HD, a large observational study of HD, leads to earlier prediction of motor-onset compared to the clinician's subjective judgment.

\section{3}

\section{Exploring the Interoceptive Awareness in Huntington's Disease} Patients

Natascia De Lucia*, Alessandro Roca, Elena Salvatore, Luigi Di Maio, Giuseppe De Michele

Reproductive and Odontostomatological Sciences "Federico II" University, Naples, Italy (all authors)

Background: Interoceptive awareness (IA) refers to the perception of physical sensations coming from one's own body that may be impaired under neurological conditions. According to the "Somatic marker" hypothesis, the body-relatedness somatic information guides emotional/ behavioral and cognitive processes.

Objective: In the present study, we aimed to assess IA in patients with Huntington's disease (HD), exploring whether IA could enhance the comprehension of cognitive dysfunctions (e.g., memory and frontal/ executive functioning) and emotion/behavior regulation.

Methods: We recruited a prospective sample of 25 symptomatic $\mathrm{HD}$ and 20 healthy controls (HC) who underwent a clinical and neuropsychological assessment, and an evaluation of IA by means of dedicated tests evaluating the awareness related to visceral and somatosensory sensations, and the heartbeat perception task. In this last test, participants were invited to concentrate on their heartbeats and counting how many beats (of her/his heart) they perceived across a specific time period.

Results: MANOVA showed significant differences in neuropsychological tests between the two groups (Wilks' Lambda $=0.08$; $p=0.002$ ). Correlation analyses showed that performance on selfawareness questionnaire was significantly associated with scores on problem behavior assessment (severity: $p=0.77$; frequency: $p=0.98)$, apathy scale $(p=0.74)$, depression scale $(p=0.60)$, anxiety scale $(p=0.96)$, and alexithymia scale $(p=0.82)$ in HD. Baseline heartbeat frequencies were not significantly different between HD and HC $(p=0.10)$, whereas $\mathrm{HC}$ had a significantly higher IA compared to HD $(p=0.04)$.

Conclusions: We found that HD patients have reduced IA, suggesting that the body-relatedness somatic information (related to IA) can play a crucial role in the emotional and cognitive processes in HD patients. 
94

Effects of Neuropsychiatric Symptoms and Implications for Measurement of Functional Status in Huntington's Disease

Jessie Gibson* ${ }^{1}$, Sheila H. Ridner ${ }^{1}$, Bethany A. Rhoten ${ }^{1}$, Mary S. Dietrich $^{1}$, Michael B. Sohn ${ }^{2}$, Daniel O. Claassen ${ }^{1}$

${ }^{1}$ Vanderbilt University Medical Center, Nashville, TN, USA

${ }^{2}$ University of Rochester Medical Center, Rochester, NY, USA

Background: Neuropsychiatric and cognitive symptoms account for substantial morbidity in Huntington's disease (HD), even in early stages, but their impact on functional status may not be captured using the Total Functional Capacity (TFC) scale. The aim of this cross-sectional, observational study was to assess how differential motor, cognitive, and neuropsychiatric symptoms impact functional status in HD using two instruments. Qualitative interviews in HD patients and caregivers were also conducted to further describe these relationships. A modified Theory of Unpleasant Symptoms was used as the guiding theoretical framework for this research. All subjects consented to the study in accordance with the informed consent regulations at Vanderbilt University Medical Center.

Methods: In 50 patients with HD, multiple regression analyses assessed the associations of neuropsychiatric, cognitive, and motor symptoms with functional status as measured using the TFC and Adult Functional Adaptive Behavior (AFAB) scales. Qualitative interview transcripts $(n=15)$ were coded using qualitative descriptive content analysis, and codes were categorized into themes. Procedures followed were in accordance with the ethical standards of the responsible committee on human experimentation (institutional and national) and with the Helsinki Declaration of 1975, as revised in 2000 (World Medical Association Declaration of Helsinki, 2000).

Results: Greater burden of neuropsychiatric, cognitive, and motor symptoms were associated with greater impairments to functional status as measured by the AFAB $(p<0.05)$. Only motor symptoms were associated with TFC. Qualitative themes from HD patients and caregiver interviews revealed broad, clinically significant perceived effects of neuropsychiatric symptoms on functional status.

Conclusions: The TFC may have limited applicability, particularly in early-stage HD patients, as a measure of functional status. This is important to consider in the design of clinical trials, as patients and caregivers reported significant perceived effects of neuropsychiatric symptoms on function, and TFC may not capture these effects. The AFAB can be used in HD studies as a more holistic measure of functional status, reflecting physical, mental, and social domains of health.

\section{5}

\section{Optimizing Nanoparticles for Intranasal Administration of Gene} Therapy of Huntington's Disease

Oksana Firhurka, J. Sanchez-Ramos*

University of South Florida, Tampa, FL, USA (all authors)

Background: Lowering HTT gene expression in patients with Huntington's disease (HD) currently requires chronic administration of antisense oligonucleotides (ASO) or small interfering RNA (siRNA) into the cerebrospinal fluid (CSF) by intrathecal infusions or direct intracerebral injections. Ongoing clinical studies have demonstrated that intrathecal administration of antiHTT ASO is well-tolerated for at least several years. However, repetitive long-term intrathecal infusions can result in a range of complications and may not be acceptable for a lifetime of treatment.
Methods: In developing a nose-to-brain nanocarrier system for gene therapy, we prepared a series of nanoparticle (NP) formulations that used chitosan or other matrix materials for encapsulating antiHTT siRNA. The NPs were designed to package the maximum amount of siRNA (an "enrichment" procedure), while at the same time protecting the payload from degradation "en route" to the target.

Results: Optimization experiments identified key factors to improve production of effective nanocarriers of antiHTT siRNA. We identified four enriched chitosan-based formulations of NPs that protected the payload in transit while not impeding release of the siRNA in the target brain tissue. All four formulations were effective in lowering HTT mRNA expression by at least $50 \%$ in the YAC128 mouse model (HD).

Conclusions: Intranasal administration of nanoparticles carrying siRNA is a promising therapeutic alternative for safe and effective lowering of mutant HTT expression.

\section{6}

VMAT 2 Inhibitor and Antipsychotic Use in Individuals with Huntington's Disease Using Enroll-HD Data

\section{Erin Furr-Stimming ${ }^{*}$, Liang Zhu, Natalia Pessoa Rocha}

The University of Texas Health Science Center at Houston, McGovern Medical School, Houston, TX, USA (all authors)

Background: Recent clinical trials have evaluated the efficacy and safety of VMAT2 inhibitors, such as valbenazine and deutetrabenazine, in individuals with tardive dyskinesia (TD) due to antipsychotic use. In most clinical trials using VMAT2 inhibitors to treat TD, study subjects remained on antipsychotics. There is little to no published data on the concomitant use of VMAT2 inhibitors (tetrabenazine and deutetrabenazine, both FDA approved for chorea in HD) and antipsychotics in individuals with HD. Due to the lack of data on potential side effects with this drug combination, there are no recommendations for their simultaneous use in HD. Therefore, this study aims to retrospectively investigate whether the concomitant use of VMAT2 inhibitors and antipsychotics are associated with worsening motor symptoms.

Methods: This is a retrospective longitudinal study including manifest HD patients from the Enroll-HD database (PDS4). The database assesses participants annually; manifest HD, with at least four years of visits, were included in analysis. Linear mixed models were used to assess motor, behavioral, and cognitive functioning over a series of visits in patients who have taken VMAT2 inhibitors (WHO ATC/DDD code $=$ N07XX), antipsychotics (WHO ATC/DDD code $=$ N05A), both or none.

Results: Participants who have taken either VMAT2 inhibitors or antipsychotics presented with worse motor scores over the four annual visits than participants who have never taken these drugs. Patients who have taken both drugs (i.e., VMAT2 inhibitors and antipsychotics) presented with worse scores since the first (baseline) visit, thus concluding that the concomitant prescription of these drugs occurred when patients presented with greater severity of motor symptoms. The use of VMAT2 inhibitors, antipsychotics, or both did not alter disease progression. Patients with HD who have used both VMAT2 inhibitors and antipsychotics had more severe motor symptoms at baseline than patients who have taken these drugs separately or who have never taken any of these drugs, these differences persisted throughout a four period. The concomitant use of VMAT2 inhibitors and antipsychotics was not associated with a worsening in motor symptoms. 
97

Tetrabenazine: Impact on Impulsivity Scales in Huntington's Disease

William Ondo* ${ }^{1}$, Chia Arif ${ }^{1}$, Bethany Williams ${ }^{2}$, Kenneth Podel ${ }^{1}$

${ }^{1}$ Methodist Neurological Institute, Houston, TX, USA

${ }^{2}$ The University of Texas Health Science Center at Houston, McGovern Medical School, Houston, TX, USA

Background: Impulsivity and poor decision making are common features of symptomatic Huntington's disease (HD). Tetrabenazine (TBZ) is a vesicular monoamine-2 inhibitor, approved to treat chorea in HD that inhibits release of dopamine and other monoamines. Impulsivity and drive toward pleasure are largely mediated by dopamine, and anecdotally, TBZ reduces impulsive behavior, an endpoint that has not been formally assessed in HD treatment trials. We evaluated a series of impulsivity scales and HD motor scales in a two-month open-label trial in HD patients with problematic chorea, not necessarily selected for impulsivity.

Methods: After initial evaluation, TBZ was titrated to a maximum of $25 \mathrm{mg}$ $3 \times /$ day over 4 weeks, and subjects returned at 8 weeks for their second assessments, which were also administered to caregivers. Assessments included the UHDRS, CGI, AEs, frontal assessment battery, Barratt Impulsivity Scale-11, Questionnaire for Compulsive Impulsive Disorders in PD, Minnesota Impulsive Disorders Interview, Problem Behaviors Assessment, Montreal Cognitive Assessment, and the Columbia Suicide Severity Rating Scale. Twelve subjects, eight male, mean age 50(11) years, enrolled. One withdrew at 5 weeks secondary to $\mathrm{AE}$ but completed all postassessments while on the drug. The final daily TBZ dose was $75 \mathrm{mg}$ (9), $50 \mathrm{mg}$ (1), $37.5 \mathrm{mg}(1)$, and $12.5 \mathrm{mg}$ (1). AEs were reported in six subjects: sedation (3), insomnia (2), akathisia, agitation, dysarthria in one each.

Results: UHDRS total motor scores improved 33.1(8.0) to 21.8(6.9), $p<0.01$ (paired T-test), mostly powered by improved chorea $17.1(3.4)$ to $8.5(4.6), p<0.01$. CGI-C was marked or very markedly improved in $8 / 12$. There was marked discordance between patient and caregiver results on impulsivity and cognitive assessments with caregiver scores usually higher and more problematic than patients. Overall, TBZ did not significantly improve these measures of impulsivity. TBZ improved chorea and was generally liked in this population. However, our assessments of impulsivity did not improve on TBZ in a population not specifically recruited for impulsivity. The difficulty administering tests that require good insight in the HD population was highlighted by the marked difference in scores on tests that were also administered to caregivers.

Conclusions: Future studies could recruit only subjects with problematic impulsivity, but endpoint determination remains problematic and may require scales developed specifically for this population.

\section{8}

Longitudinal Utility of the Montreal Cognitive Assessment and Mini Mental State Exam in Huntington's Disease

Ilex Beltran-Najera* ${ }^{1}$, Jody Corey-Bloom ${ }^{2}$, Chase Snell ${ }^{2}$, Anna Smirnova ${ }^{2}$, Paul E. Gilbert ${ }^{3}$, Jonathan L. Helm ${ }^{3}$

${ }^{1}$ University of Houston, Houston, TX, USA

${ }^{2}$ University of California, San Diego, La Jolla, CA, USA

${ }^{3}$ San Diego State University, San Diego, CA, USA

Objective: Neuropsychological assessments are useful tools for examining and tracking cognitive impairment in individuals with neurological disorders; however, a full neuropsychological battery is not realistic for most routine medical visits. The present study compares the longitudinal utility of two brief cognitive instruments in Huntington's disease (HD).
Background: Currently, the most frequently used screening instrument is the Mini Mental State Examination (MMSE). A major limitation of the MMSE is the shortage of items assessing executive function and attention. These limitations are of increased concern when assessing clinical populations characterized by frontostriatal dysfunction. The Montreal Cognitive Assessment (MoCA), a more recently developed brief measure of cognitive function, encompasses a broader range of cognitivespecific items compared to the MMSE and has been shown to have high specificity and sensitivity when characterizing HD; however, there is a lack of studies examining its efficacy in detecting cognitive alterations over time.

Methods: Individuals with a definitive diagnosis of HD and three or more testing occasions for both the MoCA and MMSE were included in analyses. The MMSE was administered to 136 patients and the MoCA to 125. Multilevel modeling was used to examine the average rate of decline on both measures across time.

Results: HD patients had an average total MoCA score of 20.65 at baseline and average total MMSE score of 24.93 at baseline. Mean rates of decline on the MoCA and MMSE were $0.36(p<0.01)$ and 0.68 $(p<0.01)$ points per visit, respectively. Education level did not predict the rate of decline but did predict performance at baseline.

Conclusions: As expected, both MMSE and MoCA scores decline significantly over time; however, a lower baseline score and moderately declining slope on the MoCA may be more characteristic of HD pathology. The tendency for ceiling and floor effects on the MMSE could possibly explain a higher average score at enrollment and steeper slope across time. The MoCA may be a more useful measure for assessing cognitive changes in HD because of its sensitivity to executive dysfunction.

\section{9}

\section{Behavioral and Executive Dysfunction in Juvenile-Onset Huntington's Disease}

Kathleen Langbehn*, Ashley Cochran, Ellen van der Plas, Amy L. Conrad, Eric Epping, Peg Nopoulos

University of Iowa Carver College of Medicine, Iowa City, IA, USA (all authors)

Background: Reports of behavioral and executive dysfunction in juvenile-onset Huntington's Disease (JOHD) have been based primarily on qualitative caregiver reports or retrospective medical record reviews. The aim of the present study was to quantify differences in behavior and executive function between patients with JOHD and unaffected participants using informant- and self-report questionnaires.

Methods: The analysis included 49 observations from 21 children/young adults (12 female) with JOHD and 203 observations from 115 children/ young adults (64 female) with a family history of Huntington's disease, but who did not inherit the disease (GNE). Informants completed the Behavior Rating Inventory of Executive Function (BRIEF) and the Pediatric Behavioral Scale (PBS) short version. All participants consented in accordance with the University of Iowa IRB. Mixed linear regression models were conducted to 1) assess if there were group differences on these measures, 2) evaluate CAG repeat effects in the JOHD group, and 3) determine group differences between informant- vs selfreports. All models were adjusted for age, sex, and random effects of participant and family relations.

Results: The JOHD group had significantly higher scores, indicating more problems, than the GNE group on all subscales of the BRIEF, and measures of Aggression/Opposition and Hyperactivity/Inattention of the PBS (all $p<.05$ ). There were no group differences in Depression/ Anxiety. Inhibition, Planning/Organization, Initiation, and Aggression/ Opposition had significant negative correlations with CAG repeat length 
(all $p<.05$ ). There was a greater discrepancy between informant- vs. selfreported scores in the JOHD group compared to GNE.

Conclusions: Significant behavioral and executive functioning differences were evident between JOHD and GNE groups, supporting prior qualitative reports of deficits in JOHD patients, though the lack of mood/anxiety symptoms in JOHD is notable. The association of CAG repeat length in JOHD indicates that patients with shorter repeats (typically adolescent-onset) report more behavioral symptoms than patients with longer repeats (typically childhood-onset). Discrepancies between informant- vs. self-reports in the JOHD group may indicate a lack of insight in JOHD patients. These results provide evidence of the behavioral and executive dysfunction characteristics of JOHD that can be used to monitor disease progression and measure efficacy of therapeutic interventions.

\section{0}

Layers of Loss: a Scoping Review of Huntington's Disease Caregivers' Spiritual Distress and Coping Strategies

Bethany Leidl* ${ }^{1}$, Debbi Fox-Davis ${ }^{2}$, Tracie L. Baker, Francis O. Walker $^{1}$, Buddy Marterre

${ }^{1}$ Wake Forest University School of Medicine, Winston-Salem, NC, USA ${ }^{2}$ HD Reach, Denver, CO, USA

Background: HD familial caregivers experience unique challenges and significant distress. In addition to physical, social, and fiscal burdens, family members are also confronted with a myriad of spiritually related stressors. Palliative care clinicians are experts in spiritual assessment and grief support and may be able to assist present teams and compassionately aid struggling HD caregivers. However, HD caregivers' spiritual stressors and coping strategies have yet to be systematically studied.

Methods: A scoping literature review was conducted using PRISMA$\mathrm{ScR}$ guidelines to assess spiritual suffering and coping mechanisms of HD caregivers. PubMED, EMBASE, CINAHL, and PsycINFO were searched for qualitative, quantitative, and mixed method studies exploring HD caregiver grief, psychological and spiritual health, well-being, suffering, and coping mechanisms. Data from relevant articles was organized thematically using induction and open coding. A preliminary, grounded, deductive approach with axial coding has tentatively identified possible relationships between major themes. More thematic analysis iterations are ongoing.

Results: 771 articles were initially identified, of which 54 were included in the final thematic analysis. These articles describe many types of losses that HD caregivers experience, such as the loss of their loved one's personhood/dignity, family structure, social connectedness, and even their sense of self. These losses appear gradually and evolve, on average over the span of 10-15 years. The majority of studies report maladaptive HD caregiver coping strategies, including not processing feelings of guilt, anger, or fear; or suffering from meaninglessness, despair, avoidance, and/or isolation. A minority of studies identified effective coping strategies that include seeking acceptance, peace, meaning-making, hope, and healthily processing anger, sadness, and fear. Coping strategies that relate to relationships with God images (including anger toward God / existential angst) and their religious community (both positive and negative) require further, ongoing, exploration, and analysis before reporting.

Conclusions: HD caregivers experience prolonged and complicated grief as they not only slowly and progressively lose their loved one but also their lives as they know it. We recommend better measurement tools to assess the many forms of spiritual loss experienced by HD caregivers so that, one day soon, they may be better supported by healthcare teams.
101

Using the Repeatable Battery for the Assessment of Neuropsychological Status (RBANS) to Detect Cognitive Change in Premanifest Huntington's Disease

Andrea Mustafa* ${ }^{1}$, Jody Corey-Bloom ${ }^{1}$, Chase Snell ${ }^{1}$, Haileigh Smith ${ }^{1}$, Jordan Castleton $^{1}$, Paul E. Gilbert ${ }^{2}$

${ }^{1}$ University of California, San Diego, La Jolla, CA, USA

${ }^{2}$ San Diego State University, San Diego, CA, USA

Objective: The objective of the current study was to examine cognitive performance in premanifest gene carriers for Huntington's disease (PreHD), individuals with manifest Huntington's disease (HD), and healthy adults (HA) using the Repeatable Battery for the Assessment of Neuropsychological Status (RBANS).

Background: The RBANS is a brief standardized screening tool that measures cognitive performance across five domains: immediate memory, visuospatial/constructional, language, attention, and delayed memory. Although previous reports have described poorer performance on the RBANS in HD subjects relative to normative data, to our knowledge, there have been no published studies utilizing the RBANS to detect early cognitive change prior to manifest disease onset.

Methods: Eighty-nine participants, including $33 \mathrm{PreHD}, 31 \mathrm{HD}$, and 25 HA were administered the RBANS using standard procedures.

Results: Results from a one-way ANOVA test and follow-up post hoc Tukey HSD test revealed a significant main effect of group in RBANS Total Scale of Index Scores (TSIS) between HD, PreHD, and HA groups $\mathrm{F}(2,86)=28.754, p<0.001$. More specifically, the HA group had significantly higher RBANS TSIS than the HD group $(p<0.001, d=1.89)$. There was no significant difference between the HA and PreHD groups in RBANS TSIS $(p=0.060)$; however, a moderate effect size suggests a trend of detectable cognitive differences between these two groups $(d=$ 0.66). Similarly, a multivariate analysis of variance and follow-up univariate tests revealed a significant main effect of group in each of the five cognitive domain index scores, with the HA group significantly outperforming the HD group in all indexes $(p s<0.001)$. Although there were no significant differences between the HA and preHD groups on most of the cognitive indexes, a moderate effect size, suggesting a trend toward detectable difference in the preHD subjects, was found for Attention $(d=0.61)$.

Conclusions: This study is consistent with previous literature demonstrating cognitive deficits in HD as compared to HA and PreHD using the RBANS. The current study, however, also provides evidence that, in addition to manifest HD differences, early cognitive change, prior to manifest disease onset, can be detected utilizing this measure.

\section{2}

Direct and Indirect Associations of Working Memory, Coping with Stress, and Symptoms of Depression and Anxiety in Patients with HD

Abigail Ciriegio*, Kelly H. Watson, Anna Pflazer, Lisa Hale, Maile T. Jones, Brittany Brown, Katherine McDonell, Daniel O. Claassen, Bruce E. Compas

Vanderbilt University, Nashville, TN, USA (all authors)

Background: Prior research has shown that Huntington's disease (HD) is marked by deficits in working memory (You et al., 2014) and elevations in psychiatric symptoms throughout the course of the disease (Epping et al., 2016). Research with offspring of HD patients has shown that an important factor in understanding the relationship between working memory and psychiatric symptoms is the use of complex cognitive coping 
strategies (e.g., cognitive reappraisal; Ciriegio et al., 2020). However, research has not yet investigated the association between these processes in adult HD patients. The current study sought to examine the associations among working memory, coping, and psychiatric symptoms in patients with HD.

Methods: The sample included 25 patients with $\mathrm{HD}$ (52\% male; $\mathrm{M}$ age $=$ 45.60, $\mathrm{SD}=9.76$ ). Working memory was assessed using the List Sorting Working Memory Task from the NIH Toolbox Cognition Battery (Gershon et al., 2010). The Patient Health Questionnaire-9 (Kroenke \& Spitzer, 2002) and Generalized Anxiety Disorder-7 (Spitzer et al., 2006) were used to assess symptoms of depression and anxiety, respectively. The Responses to Stress Questionnaire-HD version (Ciriegio et al., 2020) examined coping strategies in response to HD-related stressors. All subjects gave consent in accordance with the Vanderbilt University IRB. Direct and indirect associations were tested using PROCESS (Hayes, 2018).

Results: Analysis of the full models revealed the direct association between working memory and symptoms of depression and anxiety was not significant. The direct association between working memory and secondary control coping was significant $(\beta=.45, p=.04)$, as was the direct association between secondary control coping and symptoms of depres$\operatorname{sion}(\beta=-.62, p=.01)$ and anxiety $(\beta=-.47, p=.05)$. The total indirect effect of working memory on symptoms of depression through secondary control coping was significant $(\beta=-.28,95 \% C I(-.58$ to -.05$))$. Similarly, the indirect effect of working memory on symptoms of anxiety through secondary control coping was also significant $(\beta=-.21,95 \% C I$ $(-.49$ to -.02$))$.

Conclusions: This study provides evidence that deficits in working memory are associated with increased levels of depression and anxiety through their association with cognitive skills to cope with stress in HD patients. Findings suggest improving working memory skills and promoting complex cognitive coping strategies may be potential targets to mitigate psychiatric symptoms.

\section{3}

\section{Correlates of Observed Emotional and Behavioral Aspects of} Communication in Parents with Huntington's Disease

Kelly Watson*, Abagail E. Ciriegio, Anna Pfalzer, Lisa Hale, Maile Jones, Brittany Brown, Katherine McDonell, Daniel O. Claassen, Bruce E. Compas

Vanderbilt University, Nashville, TN, USA (all authors)

Background: Communication in families plays a central role in physical and mental health (Alm et al., 2019). The symptoms of Huntington's disease (HD) likely present challenges to effective communication, as cognitive impairments can adversely affect language, motor impairments can affect speech, and psychiatric symptoms can affect emotional and behavioral aspects of communication. Despite a rich body of qualitative research suggesting HD is characterized by disruptions in communication (Hartelius et al., 2010), no studies to our knowledge have directly observed family communication. The current study examined associations of observed emotions and behaviors of parents with HD with their neurocognitive functioning, self-reports of stress and depression, and disease characteristics.

Methods: Twenty-two parents with HD (59\% male) and offspring (M age $=19.96,37 \%$ male) participated in a video-recorded conversation about HD stress. Parents' observed emotions and communication were coded using the Iowa Family Interaction Rating Scale (Melby \& Conger, 1998). Parents completed the Patient Health Questionnaire (Kroenke \& Spitzer, 2002) to assess depressive symptoms and Responses to Stress Questionnaire (Ciriegio et al., 2020) to assess HD stress. Working memory was assessed with the NIH Toolbox (Gershon et al., 2010) and disease characteristics (i.e., CAP, disease stage) came from medical records. All participants consented to the study in accordance with the informed consent regulations at the institution.

Results: In bivariate correlation analyses, observed sadness was associated with greater depressive symptoms $(r=0.75, p<0.01)$ and HD stress $(r=0.47, p<0.05)$. Observed anxiety was related to less advanced disease $(r=-0.47, p<0.05)$, while observed hostility toward offspring was related to more advanced disease $(r=0.52, p<0.05)$. Effective communication was associated with lower CAP $(r=-0.57, p<0.05)$ and greater working memory $(r=0.67, p<0.01)$.

Conclusions: These findings extend research by obtaining observations of communication between parents with HD and offspring, and identifying correlates of observed emotions and behaviors. Emotional displays during conversations about HD were related to greater stress, depression, and disease stage. Less effective communication was associated with higher CAP scores and lower working memory skills. Future research should examine associations of parental communication with offspring's psychiatric and cognitive functioning in HD families.

\section{4}

\section{Mutant Huntingtin Is Cleared from the Brain via Active Mechanisms}

Raul Banos*1 ${ }^{1}$, Christopher Yanick ${ }^{1}$, Amirah E. Aly ${ }^{2}$, Lauren M. Byrne ${ }^{3}$, Ethan D. Smith ${ }^{1}$, Yuanyun Xie ${ }^{1}$, Stephen EP Smith ${ }^{4}$, Nalini Potluri ${ }^{5}$, Hyeongju Kim ${ }^{6}$, Ihn Sik Seong ${ }^{7}$, Edward J. Wild ${ }^{3}$, Ji-Joon Song ${ }^{8}$, Michael R. Hayden ${ }^{2}$, Amber L. Southwell ${ }^{1}$

${ }^{1}$ University of Central Florida, Orlando, FL, USA

${ }^{2}$ University of British Columbia, Vancouver, British Columbia, Canada

${ }^{3}$ University College London, London, UK

${ }^{4}$ University of Washington, Seattle, WA, USA

${ }^{5}$ Sanford Burnham Prebys Medical Discovery Institute, Orlando, FL, USA

${ }^{6}$ Daejeon Institute Science and Technology, Daejeon, South Korea

${ }^{7}$ Massachusetts General Hospital (MGH) Center for Genomic Medicine, Boston, MA, USA

${ }^{8}$ Korea Advanced Institute of Science and Technology, Daejeon, South Korea

Background: Huntington's disease (HD) is caused by a CAG trinucleotide repeat expansion within the huntingtin (HTT) gene. HTT-lowering therapeutics have shown preclinical promise and are currently undergoing evaluation in clinical trials. However, the ability to clinically assess the lowering of HTT in the brain in living patients has been challenging. We have reported that mutant HTT (mtHTT) concentration in cerebrospinal fluid (CSF) of HD patients correlates with clinical measures, such as disease burden and both motor and cognitive performance. We have also shown that lowering HTT in HD mice brains leads to a correlative decrease of mtHTT in the CSF, indicating the use of such measurement as a potential biomarker in clinical trials. Initial HTT lowering clinical trials have demonstrated dose-dependent reduction in CSF HTT, suggesting that it is measuring target engagement. However, we are unable to adequately interpret this data because it is not yet clear how HTT enters the CSF. Previous studies have shown that mtHTT is released upon induced neuronal death; with a correlation also observed between mtHTT increases and that of tau and neurofilament light chain (NfL), which are neuronal proteins associated with neurodegeneration. This suggests that passive release following neurotoxicity is responsible for deposition of mtHTT in CSF. However, we have also observed mtHTT in the CSF of mice that lack overt neurodegeneration, demonstrating that non-neurodegenerative mechanisms likely also play a role in clearance of mtHTT to CSF. Understanding this relationship is important for interpretation of HTT lowering clinical trial data. 
Methods: In this study, we investigated the mechanisms of mtHTT clearance from the brain to interpret the significance of therapy-induced CSF mtHTT alterations.

Results: We show that in addition to neurotoxic insult, secretion of HTT from CNS cells, followed by glymphatic clearance from the interstitial space, plays a part in mtHTT entering the CSF.

Conclusions: In conclusion, our data supports both passive release, as well as active clearance, of mtHTT into CSF, suggesting that its treatment-induced changes may represent a combination of target engagement and preservation of neurons. Further investigation will need to be made into the relative contribution of these mechanisms to mtHTT entering the CSF compartment over the course of disease. Additionally, our data support a combinatorial application of CSF mtHTT measurement with other neurodegenerative biomarkers to provide a clearer assessment of HTT lowering target engagement.

\section{5}

Implementation of a Patient Welcome Letter for the Huntington's Disease Clinic: Improving the Patient Experience

Laura Pesantez Pacheco*, Erin Furr-Stimming, Natalia Pessoa-Rocha, Amber McCarthy, Dylan May

University of Texas Health Science Center at Houston, Houston, TX, USA (all authors)

Background: Most ambulatory practices provide information to their first-time patients before an appointment, often referred to as the Patient Welcome Letter. Several studies across a variety of disciplines have shown a positive patient response to receiving a welcome letter. However, there are limited guidelines for content, formatting, or wording of these documents. Studies have shown that patient education materials may be of limited utility to many patients, as the readability of the information often exceeds the average reading skill level of adults in the USA. Given the high prevalence and complexity of neurological disease, neurologists must recognize the average reading skill level of our patients and take steps to overcome it. We hypothesized that the creation of a welcome letter for our multidisciplinary Huntington's Disease Clinic (HDSA Center of Excellence) that is adapted according to the patient's literacy level and current needs may improve patient experience and may positively impact compliance and indirectly clinical outcomes.

Methods: This study employed standard quality improvement methods. After the development of a first draft for the welcome letter, we analyzed its reading ease using the Flesch-Kincaid readability tests. A second draft was then created by adding content and improving literacy levels. Surveys were then administered to the Huntington's Disease Clinic patients in order to gather information regarding the perceived content of the letter, patients' understanding of the information, how useful they found it, and overall value of the letter. A final draft was elaborated by using patients' feedback and improving the letter's readability to a 9th grade level.

Results: Of the 18 patients who received this letter, $78 \%$ read the letter and $44 \%$ thought it was 'Very Useful.' The majority of patients thought the letter was easy to understand, and $61 \%$ read the letter only one time, with an average reading time of 1-5 min. Finally, $61 \%$ of the patients reported they will keep the letter for future reference.

Conclusions: Patient-centered letters are informative and improve patient-perceived readiness for clinic appointments. We developed a Huntington's Disease Clinic welcome letter that is easy to read and comprehend and encompasses all the necessary appointment details. Future efforts will be directed at expanding our study and longitudinally following clinical outcome measures in individuals who receive our HD specific welcome letter.

\section{6} Social Support and Loneliness in Huntington's Disease Patients
During the COVID-19 Pandemic

Maile Jones*, Anna Pfalzer, Lisa Hale, Kelly H. Watson, Abagail E. Ciriegio, Daniel O. Claassen, Bruce E. Compas

Vanderbilt University Medical Center, Nashville, TN, USA (all authors)

Background: Interpersonal relationships are an important element of health and illness. Close and supportive relationships are related to better health, whereas loneliness and social isolation are associated with illness and poorer health (Holt-Lunstad, 2018). The quality of interpersonal relationships of patients with Huntington's disease (HD) may be affected by symptoms of the disease that interfere with communication and interactions with others. Isolation from others may be further compounded by social distancing and "safer-at-home" practices during the COVID19 pandemic. The current study examined associations of measures of loneliness and social support with disease characteristics and COVID-19 stress in a sample of HD patients.

Methods: Eighty-two HD patients $(\mathrm{M}$ age $=47.97)$ were contacted by phone during "safer-at-home" orders (between 4/2020 and 5/2020). Participants completed the UCLA Loneliness Scale (Russell et al., 1978) and the Interpersonal Support Evaluation List (ISEL; Cohen \& Hoberman, 1983), and responded to an open-ended question about the stress related to the COVID-19 pandemic. Patient clinical demographics (i.e., age, CAG repeats, CAP-score) were obtained from the medical record. All subjects gave their consent to the study in accordance with the informed consent regulations of the institution where the research was conducted.

Results: In bivariate correlations, CAG repeats and CAP scores were significantly negatively correlated with the Total Social Support Scale and the Belongingness Subscale on the ISEL (r's range from -0.20 to $-0.24, p<0.05$ ). However, CAG repeats and CAP scores were unrelated to loneliness. Patients' reports of loneliness were significantly negatively correlated with all of the ISEL social support scales (r's range from -0.32 to $-0.50, p<0.01$ ). Reports of COVID-19 stress were significantly negatively correlated with CAP score $(r=-0.26, p<0.05)$ but were not related to reports of either social support or loneliness.

Conclusions: Our findings extend previous research regarding the association between social connectedness and poorer health in a sample of HD patients. For HD patients, feelings of social isolation and loneliness increased incrementally with worse patient disease status. The "safer-at-home" orders have also placed heightened levels of stress on HD patients. However, this burden was not related to loneliness or lower social support, perhaps because these aspects of interpersonal functioning were already impaired by HD.

References:

- Cohen, S., Mermelstein, R., Kamarck, T., \& Hoberman, H. M. (1985). Measuring the functional components of social support. In: I. G. Sarason \& B. R. Sarason (Eds.), Social support: Theory, research, and applications (pp. 73-95). The Hague.

- Holt-Lunstad, J. (2018). Why social relationships are important for physical health: A systems approach to understanding and modifying risk and protection. Annual Review of Psychology, 69, 437-458.

- Russell, D. W. (1996). UCLA loneliness scale (version 3): Reliability, validity, and factor structure. Journal of Personality Assessment, 66(1), 20-40. 
107

Concurrence of Huntington's Disease and Brain Tumors-a SingleCenter Experience

Presenter:

Chandra Shivika*, Erin Furr-Stimming

The University of Texas Health Science Center at Houston, McGovern Medical School, Houston, TX, USA (all authors)

Objective: To describe the clinical presentation and diagnostic findings in three patients with Huntington's disease (HD) who were also found to have brain tumors.

Background: Various studies have shown that there is a lower incidence of cancers in patients with Huntington's disease. Only a single case report in 1996 has previously described coexistence of Huntington's disease with a brain tumor.

Methods: Case series and review of literature.

Results: Of the three patients, two were female (patient A and B) and one male (patient C). All three had genetically confirmed HD and were manifest HD. Patient A's total functional scale (TFC) was stage I. She presented with both hypomotor and hypermotor seizures in the absence of any focal neurologic signs. Imaging revealed an expansile non-enhancing right inferior temporal lesion suggestive of a low-grade glioma versus a dysembryplastic neuroepithelial tumor (DNET); and was later confirmed to be a DNET tumor after surgical resection. Patients B and C were in TFC stage III-IV. Both were noted to have new-onset hypermotor seizures and focal cortical signs (apraxia and visual neglect respectively), which led to further clinical evaluation and radio-imaging that revealed diffusely infiltrative intracranial tumors suggestive of high-grade gliomas. Patient $\mathrm{B}$ underwent surgical resection with a confirmed tissue-diagnosis of glioblastoma multiforme (GBM). Patient C's tumor was more aggressive and not amenable to resection.

Conclusions: Neuropsychiatric manifestations of HD often mask concurrent neuro-pathologies and confound their diagnosis. Longitudinal comprehensive care is helpful in raising clinical suspicion for a non-HD cause given new focal neurologic symptoms (apraxia, visual neglect), which are typically not a part of the HD spectrum. In addition, while seizures can be seen in juvenile HD patients, adult-onset ictal events in this population warrant a thorough investigation to rule out structural causes. Future directions include understanding the genetic and environmental association between coexistence of HD and brain tumors, and studying its prevalence in this population.

\section{8}

\section{Inpatient Psychiatric Admissions in Huntington's Disease}

Sarah Moroz*, Katherine McDonell, Lisa Hale, Bruce E. Compas, Daniel O. Claassen

Vanderbilt University Medical Center, Nashville, TN, USA (all authors)

Background: Psychiatric symptoms are highly prevalent in Huntington's disease (HD) and are extremely burdensome to patients and caregivers (Thompson, 2012; Eddy, 2016). Previous research has shown that rates of psychiatric admission are higher in HD patients compared to unaffected relatives (Jensen, 1993), but there is currently little evidence regarding the overall prevalence or predictors of inpatient psychiatric admission in the HD population. Evidence-based guidelines regarding the management of HD patients in the inpatient setting are also lacking.

Methods: We performed a retrospective chart review of HD patients followed at Vanderbilt University Medical Center who were admitted to a psychiatric facility between 2008 and 2019. Clinical and demographic information were obtained from the electronic medical record. Patients who presented to the ED with psychiatric symptoms, but were not admitted, were excluded.

Results: Thirty-three out of 287 patients $(11.5 \%)$ had at least one psychiatric admission. Age at first admission ranged from 17 to 74 , with $75.8 \%$ of patients in the motor manifest stage at the time of first admission. $45.5 \%$ of admitted patients were female. The most frequent indications for admission were suicidal ideation or attempt (49.2\%), psychosis $(30.5 \%)$, and aggression (32.2\%). Mean length of stay was 8.78 days (SD 7.97), with a range of 1-32 days. $63.6 \%$ of patients were admitted more than once, with 12 being admitted twice and nine at least three times. The most common actions taken during admission were pharmacological adjustments, most frequently addition of antidepressant or antipsychotic medications. Two patients were treated with electroconvulsive therapy for refractory depression. $100 \%$ of patients followed up with neurology after discharge; $82 \%$ had psychiatric follow up.

Conclusions: HD patients are admitted to inpatient psychiatric facilities for a wide range of acute psychiatric complaints most often related to suicidality, psychosis, and aggression. Repeat admissions are common, and therapeutic interventions during admission as well as postdischarge follow up care are highly variable. HD patients present distinct challenges for inpatient treatment teams, necessitating co-management of neurological symptoms and functional decline, as well as navigating difficult placement decisions. Further research is warranted to develop an integrated, disease-specific approach to guide appropriate inpatient treatment and follow up for acute psychiatric symptoms in HD.

109

Mobile App-Based Assessment Shows that Less Physical Activity and Impaired Sleep Predict Poorer Cognitive Functioning in Premanifest and Early-Manifest HD

Brendan McLaren*1, Sophie C. Andrews ${ }^{2}$, Yifat Glikmann-Johnston ${ }^{1}$, Emily-Clare Mercieca ${ }^{1}$, Nicholas W.G. Murray ${ }^{3}$, Mark A Bellgrove ${ }^{1}$, Clement Loy ${ }^{4}$, Sean P.A. Drummond ${ }^{1}$, Julie C. Stout ${ }^{1}$

${ }^{1}$ Monash University, Melbourne, Victoria, Australia

${ }^{2}$ NeuRARandwick, Australia

${ }^{3}$ Macquarie University, Sydney, Australia

${ }^{4}$ The University of Sydney, Sydney, Australia

Background: Impaired sleep and lower physical activity levels are consistently associated with poorer cognitive functioning in the general population, and are risk factors for the development of dementias, including Alzheimer's and Parkinson's disease. Relationships between sleep, physical activity, and cognition in HD are poorly understood.

Objective: We examined how day-to-day sleep and physical activity levels relate to cognitive functioning in HD using mobile-app-based assessment in late premanifest and early-manifest HD.

Methods: Forty-two HD gene-positive participants (20 manifest, 22 premanifest) participated for eight days. They wore Fitbit One activity monitors, which measured sleep and physical activity, for seven days and nights. On the morning of the eighth day they used HD-Mobile, a fit-forpurpose smartphone app for iOS and Android, to complete two cognitive tasks, one assessing visual learning and memory, and the other, a twochoice perceptual decision-making task. We recorded accuracy and response speed for each task, and produced a hierarchical multiple regression model for each cognitive outcome variable, to assess the relationships between levels of sleep and physical activity from FitBit and cognitive functioning on HD-Mobile cognitive tasks.

Results: After removing variability associated with depression symptoms and disease burden in the first step of all models, higher levels of activity, but not sleep, predicted greater accuracy on two-choice decision-making. 
In contrast, more time spent attempting to sleep, but not physical activity, predicted poorer performance and slower response speed on the visual learning and memory task. Neither physical activity, nor time spent attempting to sleep, were related to decision-making response speed.

Conclusions: Lower physical activity across 7 days predicted poorer decision-making accuracy, whereas more time spent attempting to sleep across 7 days predicted poorer visual learning and memory performance in premanifest-and manifest-HD participants. These are the first results to capture how sleep and physical activity relate to cognitive measures in people with the HD gene expansion based on data collection in a naturalistic setting (i.e., home) rather than the laboratory or clinic. Ecologically valid monitoring of sleep, physical activity, and cognition can become a useful adjunct to treatment of people with HD by linking the effects of lifestyle and medication interventions on these important outcomes.

\section{0}

\section{Characterization of an Orally Bioavailable, Small Molecule That} Lowers Mutant Huntingtin Levels

Rajeev Sivasankaran* ${ }^{1}$, Caroline Gubser-Keller ${ }^{1}$, Youngah Shin ${ }^{1}$, Alejandro Monteys ${ }^{2}$, Martin Beibel ${ }^{1}$, Nicole Renaud ${ }^{1}$, Jang-Ho Cha ${ }^{1}$, Bev Davidson ${ }^{2}$, Beth Borowsky ${ }^{1}$
${ }^{1}$ Novartis Institutes for Biomedical Research, Cambridge, MA, USA

${ }^{2}$ University of Pennsylvania, Philadelphia, PA, USA

Huntington's disease (HD) is caused by the abnormal and pathogenic expansion of CAG trinucleotide repeats beyond 35 repeats in exon 1 of the HTT gene. Although multiple downstream mechanisms have been implicated in HD, approaches that lower mutant HTT transcript and protein have emerged as the most promising therapeutic strategy based, in part, on compelling preclinical data. To date, no disease-modifying therapies are available for HD, although RNA interference and antisense oligonucleotide approaches to lower HTT are currently being tested in the clinic. Our efforts toward identifying small molecules that lower mutant HTT protein levels have led to the identification of orally available, brain penetrant molecules that potently lower HTT transcript and protein levels. We will present data on the detailed characterization of one of these molecules, including in vitro and in vivo data demonstrating the lowering of mutant HTT in cells, as well as key regions of the brain and peripheral tissues in a mouse model of HD.

Publisher's Note Springer Nature remains neutral with regard to jurisdictional claims in published maps and institutional affiliations. 